\title{
Pakistan: Second Review and Request for the Augmentation of Access Under the Stand-By Arrangement-Staff Report; Staff Supplement; Staff Statement; Press Release on the Executive Board Discussion; and Statement by the Executive Director for Pakistan
}

In the context of the Second Review and Request for the Augmentation of Access Under the StandBy Arrangement, the following documents have been released and are included in this package:

- $\quad$ The staff report for the Second Review and Request for the Augmentation of Access Under the Stand-By Arrangement, prepared by a staff team of the IMF, following discussions that ended on July 10, 2009, with the officials of Pakistan on economic developments and policies. Based on information available at the time of these discussions, the staff report was completed on July 29, 2009. The views expressed in the staff report are those of the staff team and do not necessarily reflect the views of the Executive Board of the IMF.

- A staff supplement of August 4, 2009, providing an update of assessment of the risks to the Fund and the Fund's liquidity position.

- $\quad$ A staff statement of August 7, 2009, updating information on recent developments.

- $\quad$ A Press Release summarizing the views of the Executive Board as expressed during its August 7, 2009 discussion of the staff report that completed the review and request.

- $\quad$ A statement by the Executive Director for Pakistan.

The documents listed below will be separately released.

Letter of Intent sent to the IMF by the authorities of Pakistan* Memorandum of Economic and Financial Policies by the authorities of Pakistan* Technical Memorandum of Understanding*

*Also included in Staff Report

The policy of publication of staff reports and other documents allows for the deletion of market-sensitive information.

Copies of this report are available to the public from

International Monetary Fund • Publication Services

$70019^{\text {th }}$ Street, N.W. • Washington, D.C. 20431

Telephone: (202) 623-7430 • Telefax: (202) 623-7201

E-mail: publications@imf.org •Internet: http://www.imf.org

\section{International Monetary Fund Washington, D.C.}



INTERNATIONAL MONETARY FUND

\title{
PAKISTAN
}

\section{Second Review and Request for the Augmentation of Access Under the Stand-By Arrangement}

\author{
Prepared by the Middle East and Central Asia Department \\ (In consultation with other departments) \\ Approved by Juan Carlos Di Tata and Michele Shannon
}

July 29, 2009

- Discussions were held during May 4-11, 2009 in Dubai and July 3-10 in Istanbul.

The staff met with Finance Advisor Tarin, State Bank of Pakistan Governor Raza, Chairman Pasha of the Prime Minister's Economic Advisory Council, Finance Secretary Siddique, and other senior officials. The IMF team consisted of Messrs. Mazarei (Head), Wieczorek, Kock, and Ms. Morsy (all MCD), Mr. Sun (SPR), Messrs. Keen, Krelove, and Westphal (all FAD), and Mr. Ross (the Fund's resident representative in Pakistan). Ms. Kahkonen and Mr. Silvani (World Bank) and Mr. Parvez (Asian Development Bank) participated in the discussions on structural issues. Mr. Ahmad (OED) also participated in the discussions.

- On November 24, 2008, the Executive Board approved a 23-month Stand-By Arrangement (SBA) for Pakistan under the Emergency Financing Mechanism in the amount of SDR 5.168 billion, or 500 percent of quota.

- In the attached Letter of Intent, the authorities request completion of the second review under the SBA and augmentation of access by SDR 2,067.4 million (200 percent of quota). The augmentation is justified because of increased balance of payments needs due to increased risks to the external outlook. A portion of the augmented access, equivalent to SDR 951.1 million or 92 percent of quota, would be used to finance priority government spending until Pakistan receives financial support for 2009/10 pledged at the Tokyo donor conference in April 2009. The authorities also request the extension of the arrangement through end-2010 and the rephasing of purchases.

- Program performance has been mixed. All end-March quantitative performance criteria were met, but structural reform has been slower than programmed. Final data needed to assess program performance in the last quarter of 2008/09 were not available before the issuance of this report. Preliminary information suggests that the end-June floor for net foreign assets of the central bank and the ceilings for net domestic assets of the central bank and government borrowing from the central bank were met; however, the authorities indicated that the fiscal deficit ceiling may have been exceeded by 0.9 percent of GDP and, therefore, request a waiver of nonobservance for the related end-June 2009 performance criterion. The authorities also request waivers of applicability for other end-June quantitative performance criteria and waivers of nonobservance for the two structural performance criteria for end-June. 


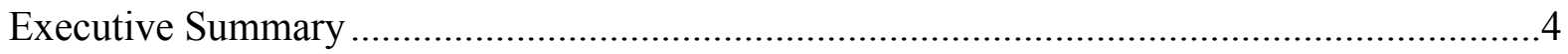

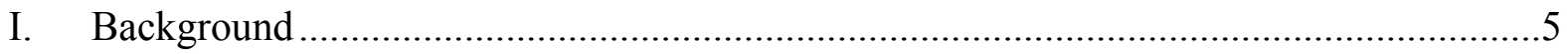

II. Recent Economic Developments ............................................................................

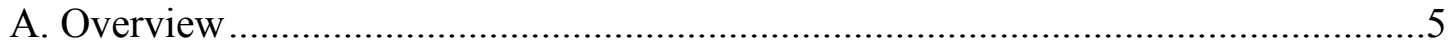

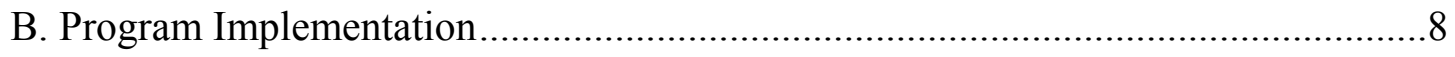

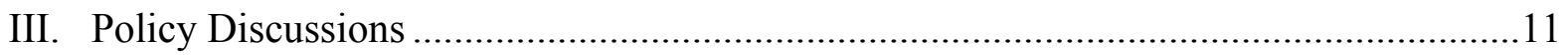

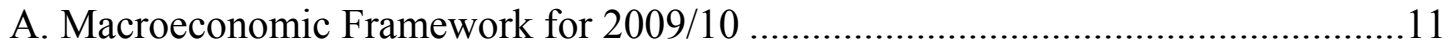

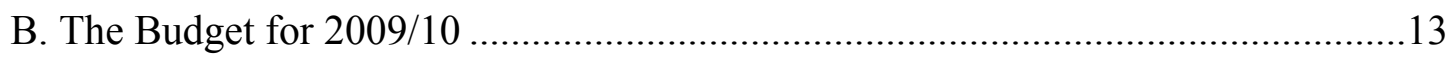

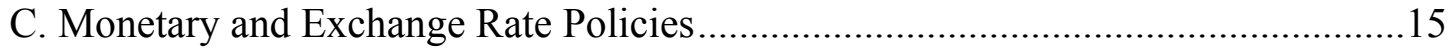

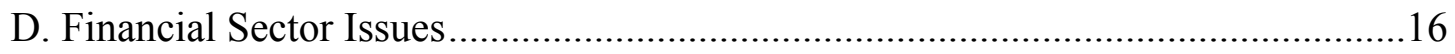

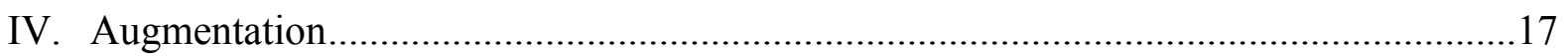

V. The Medium-term Outlook and Debt Sustainability .............................................18

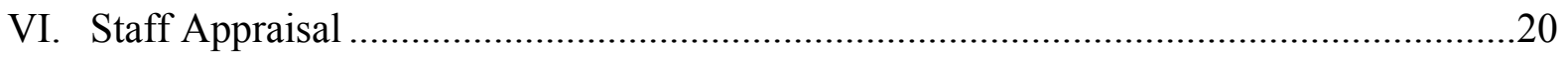

\section{Boxes}

1. Restoring the Financial Viability of the Electricity Sector ....................................... 10

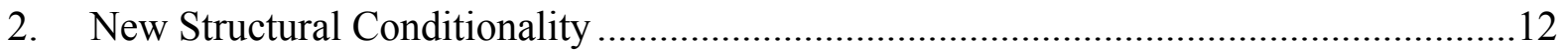

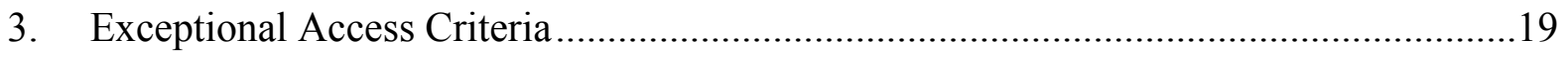

\section{Figures}

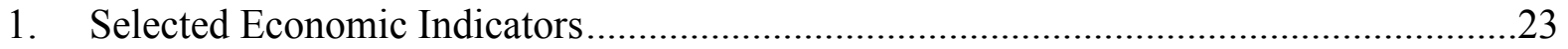

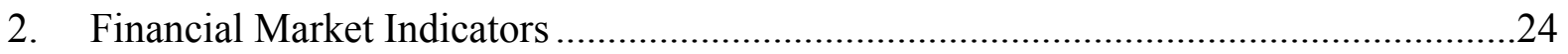

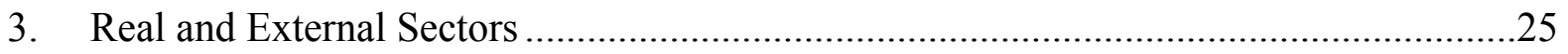

4. Fiscal Policy Indicators, 2007/08-2009/10 ................................................................26

\section{Tables}

1. Selected Economic Indicators, 2007/08-2009/10 .....................................................27

2. Balance of Payments, 2007/08-2009/10 ................................................................28

3a. Consolidated Government Budget, 2007/08-2009/10 (in billions of Pakistani rupees) 29

3b. Consolidated Government Budget, 2008/08-2009/10 (in percent of GDP)...................30

4. Monetary Survey and Analytical Balance Sheet of the State Bank of Pakistan,

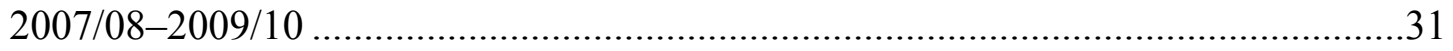

5. Medium-Term Macroeconomic Framework, 2007/08-2013/14 .................................32

6. Medium-Term Balance of Payments, 2007/08-2013/14 ...............................................33

7. Medium-Term Fiscal Framework, 2007/08-2013/14 ...............................................34

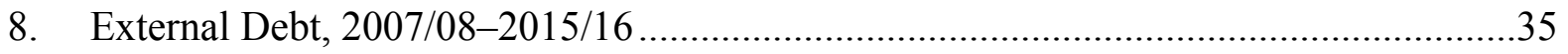


9. Gross Financing Requirements and Sources, 2008/09-2010/11 …...............................36

10. Indicators of Fund Credit, 2008/09-2015/16 ..............................................................

11a. Accessing and Phasing Under the Proposed Augmented Stand-By Arrangement,

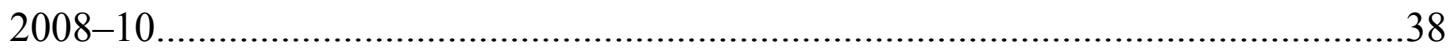

11b. Access and Phasing Under the Proposed Augmented Stand-By Arrangement,

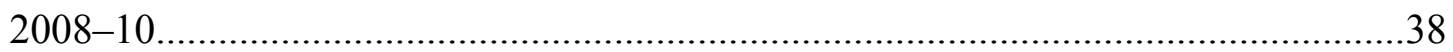

12. Selected Vulnerability Indicators, 2007/08-2013/14 ................................................39

13. Financial Soundness Indicators for the Banking System...............................................40

\section{Appendix}

Debt Sustainability Analysis.

\section{Attachments}

1. Letter of Intent 45

2. Second Supplementary Memorandum of Economic and Financial Policies

3. Technical Memorandum of Understanding 


\section{EXECUTIVE SUMMARY}

The volatile political and security situation has undermined economic confidence and complicated policy making. Reforms in the financial sector and the foreign exchange market have been progressing broadly as envisaged, and steps have been taken to strengthen the social safety net. Revenue mobilization efforts, however, have proven inadequate, as tax policy and tax administration reforms have been delayed.

Despite shortcomings in policy implementation, Pakistan's economy has continued to stabilize. Growth has been anemic and the near-term outlook for economic activity remains weak. Inflation has continued to decline, but remains persistent. With imports contracting sharply and workers' remittances continuing to grow, Pakistan's external current account position has improved. But, the financial account remains weak.

While most elements of the quantitative program have remained broadly on track, the endJune fiscal deficit target was missed by a large margin and the implementation of structural conditionality needs to be strengthened. All quantitative performance criteria for end-March were observed. Both structural benchmarks for end-March 2009 were also met (with some delay in one case). Given data lags, program performance through end-June cannot yet be fully assessed. Preliminary information indicates that most quantitative performance criteria for end-June were met, but the budget deficit target was most likely missed. The authorities, therefore, request a waiver of nonobservance for the related performance criterion and waivers of applicability for other end-June quantitative performance criteria. The structural conditionality through end-June was not met, including two structural performance criteria, also requiring waivers; the related program commitments have been converted into structural benchmarks for the third review.

Looking ahead, the authorities renewed their commitment to press ahead with the program, especially the introduction of a broad-based VAT to correct the structural shortcomings in Pakistan's tax system, which relies on a very narrow tax base. The introduction of a broad-based VAT in mid-2010 is a key pillar of their fiscal strategy.

The authorities aim to address the problems in the electricity sector. A plan has been prepared to eliminate the stock of "circular" debt in the energy sector. The government has agreed with World Bank and Asian Development Bank (ADB) staffs on a plan to increase electricity tariffs in the course of 2009/10 and eliminate tariff differential subsidies in 2010/11.

To allow for greater social, development, and security spending, donors have made significant financial pledges to Pakistan. Donor support has created room for relaxing the fiscal deficit target for 2009/10, while maintaining the course of economic stabilization.

To deal with the increased balance of payments needs, the authorities have requested an augmentation of access by 200 percent of quota. A portion of Fund credit would be used to finance much needed social spending in 2009/10 before the pledged donor support is disbursed, recognizing the difficulty for Pakistan to fund this spending domestically without increasing the risks to the external position. Given the authorities' renewed commitment to the program despite a very difficult security and political situation, staff supports their requests for the waivers, extension of the arrangement through end-2010 and rephasing of purchases, and recommends the completion of the second review and the approval of augmentation of access. 


\section{BACKGROUND}

1. The volatile political and security situation has undermined economic confidence and complicated policy making. The military operations in the Swat Valley have resulted in nearly three million internally displaced persons (IDPs). The security situation also remains grave in other parts of the North West Frontier Province, Baluchistan, the tribal areas, and major cities. Looking ahead, supporting IDPs and reconstruction in the areas affected by military operations will be a major challenge.

2. To allow for greater social, development, and security spending, donors have made significant financial pledges to Pakistan. The Friends of Democratic Pakistan Meeting and Donor Conference held in Tokyo in April mobilized pledges of $\$ 5.7$ billion, of which about $\$ 2$ billion (1.1 percent of GDP) - mostly in the form of project loans - is expected to be disbursed in 2009/10. The authorities also expect that additional budgetary grants (amounting to about 0.3 percent of GDP) would be available to support spending on IDPs. Meanwhile, the bulk of donor support for IDP-related spending has been delivered outside the budget.

3. Donor support has created room for relaxing the fiscal deficit target for 2009/10, while maintaining the course of macroeconomic stabilization. Accordingly, the authorities and staff have agreed that the 2009/10 deficit target (excluding grants) could be increased to 4.6 percent of GDP, compared to the original program target of 3.4 percent of GDP, to accommodate spending expected to be financed with disbursements of Tokyo pledges. Based on the assumption of additional budgetary grants for IDPs, the authorities increased the fiscal deficit target (excluding grants) to 4.9 percent of GDP, as reflected in the budget approved by parliament.

4. The authorities have requested an augmentation of access by 200 percent of quota. A portion of the augmented access, equivalent to SDR 951.1 million (\$1.4 billion), or 92 percent of quota, could be used to finance priority spending until the disbursements of donor support pledged for 2009/10 are received.

\section{RECENT ECONOMIC DEVELOPMENTS}

\section{A. Overview}

\section{Output and Inflation}

5. Growth has been anemic and the near-term outlook for economic activity, especially manufacturing, remains weak. A rebound in agriculture on the back of a bumper wheat crop helped maintain growth in positive territory. However, with increasing weakness in large-scale manufacturing, exports, and private sector credit, the estimate of real GDP growth for 2008/09 has been lowered from 2.5 to 2.0 percent. Moreover, the Federal Bureau of Statistics recently revised down real GDP growth for 2007/08 from 5.8 to 4.1 percent, indicating that the economic slowdown began before 2008/09. 
6. Inflation has continued to decline, but core inflation remains high and persistent. With the price surge observed in the first half of 2008 now fully reflected in the base period CPI and the sharp contraction of bank credit growth to the private sector, headline inflation (year-on-year) declined from a peak of 25 percent in October 2008 to 13.1 percent in June. However, the pace of disinflation in the latter part of 2008/09 was somewhat slower than expected, mainly on account of a surge in food price inflation in February-April, as well as a higher government procurement price for wheat. Meanwhile, core inflation fell from 18.9 percent in November 2008 to 15.9 percent in June.

\section{Money and Finance}

\section{Monetary growth has been low reflecting weak private demand for credit, but} public sector borrowing has been high. ${ }^{1}$ The $100 \mathrm{bps}$ cut in the policy rate (SBP discount rate) in April was widely anticipated and well-received by the market. The liquidity overhang, which emerged in the last quarter of 2008, was absorbed in the first quarter of 2009, and the key short-term interest rates are now within 200 bps of the policy rate. Banks have continued to show strong preference for treasury paper and the government was able to place its T-bills, investment bonds, and other instruments, including Term-Finance Certificates that were used to clear a portion of the "circular" debt. ${ }^{2}$ Nonbank budget financing (mostly through the National Saving Scheme) has been higher than programmed.

8. The SBP has allowed greater exchange rate flexibility in recent months, and gross official reserves rose from $\$ 3.5$ billion at end-October 2008 to $\$ 9.1$ billion at end-June 2009 (three months of next year's imports). This amount exceeds the program's target by $\$ 600$ million despite lower than anticipated disbursements of balance-of-payments support (including from the Fund), and a reduction of $\$ 300$ million in the net forward position. However, official reserves subsequently dropped to just below $\$ 8.4$ billion as of July 20 due mainly to a temporary pick-up in official payments.

9. The slowdown in economic activity continues to affect bank soundness. Financial soundness indicators through end-March show that banks remained profitable and well-capitalized but experienced a significant increase in nonperforming loans (NPLs). The risk-weighted capital adequacy ratio rose from 12.2 percent at end-December to 12.9 percent at end-March due to higher eligible capital and lower risk-weighted assets resulting from the increased share of lending to the public sector. At the same time, NPLs rose from 9.1 to 11.5 percent of gross loans. The increase in NPLs affected all categories

\footnotetext{
${ }^{1}$ In addition to somewhat larger bank financing for budget support, the increase in public sector borrowing reflected (i) high bank financing for domestic wheat procurement (Rs 50 billion higher than earlier projected due to a large crop); and (ii) higher credit to public sector enterprises (resulting inter alia from the partial resolution of the circular debt).

${ }^{2}$ The term "circular" debt refers to the inter-corporate debt in the energy sector and debt of energy companies to commercial banks accrued as a result of electricity tariffs being kept at below cost-recovery levels, collection problems, technical losses, and payment arrears to suppliers (see Box 1).
} 
of banks, but was most pronounced among public sector commercial banks, whose NPLs rose from 12.5 to 17.5 percent of gross loans.

10. Financial market indicators show a mixed picture. Pakistan's sovereign spreads have declined but still remain high, at about 1,050 bps. After a three-month rally through mid-April, the stock market has stabilized. Since mid-April, the KSE index has been fluctuating around 7,200 points, about 20 percent above its 12-month low registered in January 2009, but about 70 percent below its 12-month high registered in July 2008. There has been a slight pickup in foreign interest in Pakistani equities in recent months. The National Investment Trust State Enterprise Fund (NITSEF), created to invest in eight eligible stocks of state-owned companies, with a careful timing of purchases, has not perturbed the functioning of the market. As of June 30, the NITSEF had invested just over half of its resources amounting to Rs. 20 billion. The NITSEF website states its investment strategy and discloses financial statements and details of security purchases each quarter.

\section{Balance of Payments}

11. With imports contracting sharply and workers' remittances continuing to grow, Pakistan's external current account position has improved. Despite lower exports, the current account deficit is estimated to have declined by $\$ 5$ billion (3 percentage points of GDP) in 2008/09 due to a significant decline in imports, higher workers' remittances, and increased support from the United States (for military expenses). During the period July 2008-May 2009,

- $\quad$ exports declined by 2 percent relative to the same period of the previous yeara 5 percent drop in traditional exports such as textiles outweighed the pick up in nontraditional exports;

- $\quad$ imports dropped by about 10 percent on account of a lower oil bill and significantly lower imports of telecom equipment, motor vehicles and parts, and other goods; and

- $\quad$ workers' remittances rose by nearly 20 percent.

12. As in many emerging markets, the financial account remains weak. With the global economic slowdown and political and security uncertainties, net financial flows declined by nearly $\$ 3$ billion compared to 2007/08. FDI inflows, at $\$ 3$ billion, were $\$ 2$ billion lower and there was a net portfolio outflow of about $\$ 1.1$ billion compared to a small inflow in 2007/08. Official lending was somewhat lower than expected, reaching \$4.6 billion ( $\$ 1.6$ billion higher than in 2007/08).

\section{Public Finance}

13. Despite continuing revenue weakness, budget execution through end-March was prudent, but the end-June fiscal deficit target is likely to have been missed. 
- $\quad$ The collection of tax revenues by the Federal Board of Revenue (FBR) in 2008/09 fell short of the first SBA review projection by 1.2 percent of GDP, mainly owing to declining imports and slowing domestic demand. The revenue weakness also reflected a delay in tax administration reforms, which were subject to legal challenges. Also, the envisaged strengthening of taxpayer audits has not commenced. The weakness of FBR revenues was partially compensated by higher-thanprogrammed revenues from the petroleum development levy (PDL), despite a reduction of the PDL in May in response to Supreme Court comments.

- $\quad$ The impact of the revenue shortfall was offset by expenditure restraint, and the end-March fiscal deficit target was met. The authorities re-prioritized development expenditures, while protecting social safety net spending.

- $\quad$ Partial data for the last quarter of 2008/09 suggest that the end-June fiscal deficit target was exceeded by about 0.9 percent of GDP. Besides the continuing weakness of tax revenues, this reflected a surge in spending by provinces, as well as pressures to step up security- and IDP-related expenditures.

\section{B. Program Implementation}

\section{Quantitative performance}

\section{Despite mixed policy implementation, all quantitative performance criteria for} end-March were observed. The net foreign assets target was met with a sizeable margin, but the fiscal deficit target was met with difficulty.

15. Performance through end-June cannot yet be fully assessed. Preliminary information indicates that the performance criteria on the SBP's net foreign and domestic assets and SBP credit to the budget were met, but the budget deficit target was missed by a significant margin.

\section{Structural reform}

16. Both end-March structural benchmarks have been completed, albeit with a delay in the case of the comprehensive plan for eliminating the inter-corporate circular debt.

- In collaboration with the World Bank, the government developed by end-March a strategy and a time-bound plan to strengthen the social safety net. However, owing to limited administrative capacity, the rollout of the reformed Benazir Income Support Program (BISP) based on a poverty scorecard system will take longer than expected. Under the old BISP, 1.75 million families were approved at end-June, a number smaller than the original target of three million, and BISP disbursements in 2008/09 were lower than budgeted. However, BISP disbursements in 2009/10 are expected to increase substantially as the number of beneficiary families is targeted to increase to five million and eligible families will receive benefits for the entire year, independent of the timing of the approval of their eligibility (see $\$ 18$ of the Second Supplementary 
Memorandum (SSMEFP)).

- $\quad$ Agreement was reached between government and World Bank and ADB staffs on July 15, 2009 providing, among others, for the assumption of electricity sector debt by the government (see third bullet of $\mid 17$ ). ${ }^{3}$ Full and timely implementation of the plan should eliminate the "circular" debt and prevent its re-occurrence.

17. Structural conditionality for end-June was not met, but corrective measures have been agreed.

- $\quad$ The structural performance criterion on the submission to parliament of legislative amendments to harmonize the income tax and GST laws, for tax administration purposes, and reduce exemptions for both taxes was not observed. As a corrective measure, the authorities will submit to parliament by end-September 2009 legislative amendments to harmonize the sales tax, income tax, and federal excise tax laws with a view to facilitating tax administration (structural benchmark). However, meaningful progress in reducing tax exemptions will be achieved only with the introduction of the new broad-based VAT regime, which is scheduled to become effective on July 1, 2010 (see $\mid 28)$.

- $\quad$ The submission to parliament of amendments to the banking companies' ordinance (structural performance criterion) to enhance the SBP's enforcement powers in banking supervision has also been delayed. The authorities will submit this legislation to parliament by end-August 2009 (structural benchmark—see 938 ).

- $\quad$ The elimination of electricity tariff differential subsidies, originally scheduled for end-June 2009, has been postponed by one year (Box 1), In response to political pressures, the government backed off from an agreement with World Bank and ADB staffs on electricity tariff adjustments in the first quarter of 2009/10 that would have sufficed to eliminate tariff differential subsidies in the current fiscal year. However, on July 15, 2009, the government reached a revised agreement with World Bank and ADB staffs on a plan to increase electricity tariffs in the course of 2009/10 that would attain cost recovery in August 2010. While eliminating the need for tariff differential subsidies in the 2010/11 budget, this agreement implies the need for tariff differential subsidies in 2009/10 in the amount of Rs. 55 billion (0.4 percent of GDP). It also provides for a de-politicized regular tariff adjustment mechanism and the assumption of electricity sector debt (1.9 percent of GDP) by the government.

\footnotetext{
${ }^{3}$ In February 2009, the government had prepared a plan to reduce "circular" debt. However, reflecting insufficient electricity tariff adjustments in 2008/09 and with electricity companies' balance sheets being burdened by debt contracted on account of nonpayment by the government of electricity tariff differential subsidies, circular debt (net) rose by 0.3 percent of GDP between April and June 2009.

${ }^{4}$ The finalization, in close cooperation with the World Bank, of a schedule for electricity tariff adjustments with a view to eliminating tariff differential subsidies by end-June 2009 was a structural benchmark for end-December 2008. While a schedule for tariff adjustments was agreed upon in January 2009, it became apparent in May that, to eliminate tariff differential subsidies, higher tariff increases would be required owing to the surge in furnace oil prices and increases in other cost components.
} 
Box 1. Restoring the Financial Viability of the Electricity Sector

Despite earlier restructuring attempts and the establishment of a regulatory agency, the financial situation of Pakistan's electricity sector remains weak. This reflects insufficient tariff adjustments, nonpayment by the government of tariff differential subsidies, low collection rates in some regions (e.g., in the Federally Administered Tribal Areas (FATA) due to the security situation), and weaknesses in governance. Moreover, with frequent power outages and high tariffs paid by industrial producers on account of cross-subsidization between consumer groups, the power sector is a drag on growth. Substantial new investment is required to ensure that generation capacity keeps pace with demand, but private investors are reluctant to invest owing to unsustainable sector financing.

To restore the financial viability of the sector, the authorities had committed under the SBA to eliminate electricity tariff differential subsidies by end-June 2009. Reflecting rising fuel costs and the decision to refrain from adjusting tariffs, electricity subsidies rose to 1.3 percent of GDP in 2007/08 and contributed (together with rising fuel subsidies) to an increasingly unsustainable fiscal position. In the 2008/09 budget the authorities stated their intention to phase out electricity tariff differential subsidies by end-June 2009 and in November 2008 they increased average tariffs by 18 percent. In January 2009, the authorities announced their intention to raise tariffs by 4 percent in the first half of 2009 to phase out these subsidies by end-June 2009. However, tariffs were only raised by 1 percent in February.

In the face of a perceptible increase in fuel and nonfuel cost components, the authorities reached an agreement with World Bank and ADB staffs on May 21, 2009 on higher tariff increases. Revised calculations of the Pakistan Electric Power Company (PEPCO) had suggested that the required tariff increase would be between 17.5 and 41.5 percent, depending on the degree of load shedding, the extent of replacement of imported furnace oil with (less expensive) gas in electricity generation, and the assumption of electricity sector debt by the budget. According to this agreement, electricity tariffs were to be raised to eliminate tariff differential subsidies in 2009/10 and more gas would be used for electricity generation.

However, in the aftermath of the difficult parliamentary debate on the budget, the authorities considered the agreed tariff increases as politically too costly. In particular, they decided that the bulk of the required tariff increases would need to be postponed until after load-shedding had been reduced through additional generation capacity coming on stream.

On July 15, 2009, the authorities reached a revised agreement with World Bank and ADB staffs on a schedule for tariff increases that postpones the elimination of tariff differential subsidies to 2010/11.

According to this agreement, electricity tariffs will be raised in three stages with a view to limiting tariff differential subsidies to Rs. 55 billion in 2009/10. Most importantly, the authorities agreed to regularize and de-politicize tariff adjustments by amending the National Electric Power Regulatory Authority (NEPRA) Act through a presidential ordinance by end-July 2009 to ensure (i) monthly tariff adjustments reflecting changes in the price of imported fuel/power purchased; and (ii) quarterly adjustments reflecting changes in all other cost components, with the notification of new tariffs being issued within 15 days from NEPRA determination. Monthly adjustments will start in August 2009 and quarterly adjustments in August 2010.

The agreement also provides for the assumption by the government of electricity sector debt in the amount of at least Rs. 277 billion (1.9 percent of GDP). About Rs. 216 billion of this amount reflects bank borrowings by power companies owing to insufficient tariff increases and nonpayment of tariff differential subsidies during 2004/05-2008/09, with another Rs. 61 billion reflecting FATA receivables and past arrears of the Karachi Electric Supply Company (KESC) to power companies. This debt will be transferred to a newly created state-owned holding company and its service will be assumed by the budget. The government is committed to completing an audit of these liabilities prior to their assumption. 
18. The transition to the Treasury Single Account (TSA), a structural benchmark, is not yet complete. Significant progress was made in October 2008, when the authorities introduced a system preventing the accumulation of unspent balances in accounts outside the Federal Consolidated Fund. However, significant unspent balances remain in accounts at commercial banks, implying that the transition to a TSA is not yet complete. Following World Bank technical assistance in July 2009, the remaining balances with commercial banks will be surveyed. Identified government balances with commercial banks will be transferred to the TSA by end-June 2010, subject to an assessment of the impact on the banking sector's liquidity.

19. After extensive discussions, the authorities have reached understandings with World Bank staff on the organization of a functionally structured tax administration. Under the new structure, the responsibilities for sales, income, and excise taxes will be consolidated in one domestic revenue department. To reduce the risk of renewed legal challenges, a new occupational group encompassing the staff of the newly created department will be created within the FBR. The new FBR management structure was put in place as of June 30, 2009 (by an Executive Order of the FBR Chairman) and the functional reorganization of the FBR, including the revision of the structures of Regional Taxpayer Offices (RTOs) and Large Taxpayer Units (LTUs), will be completed by end-September 2009. The government is expected to approve the required regulations by September 15, 2009 (structural benchmark-SSMEFP, $\uparrow 17$ and Box 2).

20. The earlier intensification of exchange restrictions has been reversed. The SBP has removed all cash margin requirements on letters of credit introduced before the program. The intensification of such requirements earlier this year had resulted in the nonobservance of the continuous performance criterion against imposing or intensifying exchange restrictions.

\section{Policy Discussions}

\section{A. Macroeconomic Framework for 2009/10}

\section{Output and Inflation}

21. Staff has revised down its real GDP growth projection for 2009/10 from 4 percent to 3 percent. The expected modest recovery in 2009/10 from the estimated outcome for 2008/09 takes into account, on the one hand, weaker global growth in 2009 and, on the other, the expectation of improved supply conditions in the energy sector and the relaxation of the fiscal stance, including a significant increase in development spending. Over the medium term, growth is expected to recover gradually, reaching 6 percent a year by 2014/15, the average rate registered during 2001-08. 


\section{Box 2. New Structural Conditionality}

\section{Prior Action}

By end-July 2009-Amendment by a presidential ordinance of the NEPRA Act to ensure (i) monthly determination by NEPRA of the fuel adjustment surcharge in line with international fuel prices, with automatic implementation upon determination; and (ii) quarterly determination of overall electricity tariffs by NEPRA and notification of the adjusted tariffs within 15 days. Any review requests of overall tariffs will be completed within 15 days; if this is done within this period, the results of the review will be taken into account in the subsequent determination.

Macroeconomic relevance: In the absence of a de-politicized mechanism for regular electricity tariff adjustments, sizable financial imbalances in the electricity sector are likely to resurface.

\section{Structural Benchmarks}

By September 15, 2009-(i) Government approval of regulations to form new occupational groups within the FBR and revise the structures of Regional Taxpayer Offices and Large Taxpayer Units; (ii) Amendment of all relevant laws and rules.

Macroeconomic relevance: Tax administration reform will broaden the tax base and improve tax efficiency and collection. It is an indispensable component of the tax reform needed to achieve fiscal sustainability, and for introducing the VAT.

By end-2009-Submission of the VAT law to Parliament.

Macroeconomic relevance: The adoption of a broad-based VAT is the cornerstone of the revenue effort over the medium term, and a crucial component of the authorities' strategy to finance the needed increase in public investment without recourse to external borrowing or crowding out domestic private investment.

22. Inflation is still expected to decline to single-digits and to drop further over the medium term. This outlook, however, is subject to risks. The disinflation momentum could be endangered by higher oil prices, electricity tariff increases, higher wages, and the fiscal expansion. Accordingly, a more active monetary policy may be needed to manage inflationary pressures.

\section{Balance of Payments}

23. The current account deficit is expected to decline moderately from 5.1 percent of GDP in 2008/09 to 4.7 percent in 2009/10. A higher oil bill is projected to be more than offset by a further slowdown in non-oil imports (reflecting weak GDP growth), while exports, workers' remittances and other current account transfers are likely to decline as the prospects for a global recovery remain uncertain.

24. The capital and financial account surplus will likely be insufficient to finance the current account deficit. Official flows are projected to be $\$ 3.3$ billion higher than in 2008/09, boosted by Tokyo-related disbursements and the increased SDR allocation, while private 
capital inflows, while broadly unchanged, are subject to large risks. As a result, the overall balance of payments is expected to post a deficit, while gross reserves are expected to increase due mainly to Fund disbursements.

\section{B. The Budget for $2009 / 10$}

25. The budget was the central issue for the second review. Parliament approved the budget on June 25, after considerable debate, which brought to the fore significant resistance against additional revenue measures. ${ }^{5}$ The introduction of the carbon surcharge, although ultimately passed, proved particularly contentious. ${ }^{6}$ The budget features a deficit of 4.9 percent of GDP, compared with 4.6 percent agreed in May, to allow for higher support for IDPs. The authorities expect that the increased financing needs will be met by additional IDP-related external grants, and intend to limit the deficit to 4.6 percent of GDP if IDP-related grants are not forthcoming. The authorities recognize that the additional donor support from Tokyo provides only temporary room for meeting critical spending needs, and that the tax reform effort should be accelerated to ensure a sustained increase in revenue over the medium term. The authorities also agreed that if Tokyo-related disbursements are delayed or fall significantly short of expectations, the deficit target would be reduced. ${ }^{7}$

\section{The approved budget for 2009/10 reflects the following priorities:}

- Efforts to bolster revenue. While the budget provides for more than a dozen tax policy measures, the yield of many of them is modest. The most important measures are the introduction of (i) a carbon surcharge (later renamed as "Petroleum Levy") on petroleum products (to replace the PDL); and (ii) excise taxes on a number of services (e.g., banking services and insurance). Moreover, the budget provides for increases in the rates for (i) the withholding tax on imports; ${ }^{8}$ (ii) the excise tax on cigarettes; and (iii) the capital value tax on real estate (a transaction tax). However, the yield of these

\footnotetext{
${ }^{5}$ As policy discussions were held in two parts, understandings reached in early May with regard to the parameters of the 2009/10 budget formed the basis for the preparation of the budget that was submitted to parliament in mid-June. The discussions held in July focused on assessing the budget as approved by parliament and identifying contingency measures that would assure achievement of the deficit target.

${ }^{6}$ The legality of the carbon surcharge was challenged in early July and the surcharge was suspended by the Supreme Court. However, the surcharge was replaced by a Petroleum Levy, introduced through a presidential ordinance. There are legal challenges to this levy that will be considered by the Supreme Court in August.

${ }^{7}$ The deficit will be reduced by up to 0.4 percent of GDP if the shortfall in Tokyo-related disbursements exceeds 0.8 percent of GDP-see $\uparrow 41$.

${ }^{8}$ Pakistan maintains a large number of withholding taxes, including on imports (the withholding tax on imports is adjustable for industrial importers). In the authorities' view, the system of withholding taxes is supposed to compensate for the weak (direct) collection of corporate income tax. Staff has emphasized the need to accelerate tax administration reform, tax audits, and enforcement with a view to eliminate the need for such "second best" solutions. While minor in scale, the measure on the withholding on imports, which partially reverses a recent cut, runs counter to the objective of reducing import restrictions and should be reversed.
} 
measures ( 0.4 percent of GDP) falls short of the targeted increase in the tax-to-GDP ratio under the original program ( 0.6 percent).

- $\quad$ On the expenditure side, the budget aims to (i) provide adequate resources for IDPs; (ii) increase development spending (partially funded by donor support pledged at Tokyo); (iii) support several key industries through subsidies, including for exports; and (iv) a general increase in civil servants' salaries by 20 percent (while wages for security forces involved in the anti-terrorism campaign are to be doubled).

\section{Discussion on revenue policies}

27. The authorities believe that additional revenue measures are politically infeasible in the near term. In this context, they emphasized that they needed to preserve their political and institutional capital for the introduction of the VAT. Staff acknowledged this point but indicated that, to reduce vulnerability and provide resources for needed outlays, and because additional donor support would only be available for limited period, a strong revenue effort was needed. Staff also pointed to the significant risks related to the rather optimistic revenue projections and the effects of the delays in tax administration reform, which could undermine revenue performance early in the current fiscal year. However, given the significant resistance to revenue measures in the parliamentary debate of the budget, the authorities felt that submitting additional revenue measures would be difficult and could only be done, if necessary, in the context of a supplementary budget later in the year.

\section{The authorities renewed their commitment to press ahead with the introduction of} a broad-based VAT in 2010/11. They recognized that time is of the essence and emphasized that the introduction of a broad-based VAT in mid-2010 was a key pillar of their medium-term fiscal strategy. To help build a consensus for the VAT within the government and discuss options for its introduction, the authorities will organize a high-level conference in September with federal and provincial participation. To ensure its timely introduction, the authorities will prepare by end-September 2009 a detailed time-bound action plan for VAT implementation. A draft VAT law will be submitted to parliament by end-December 2009 (structural benchmark-SSMEFP, $₫ 16$ ).

\section{Discussion on expenditure policies}

\section{The budget provides Rs. 50 billion (0.3 percent of GDP) for IDP assistance.}

The-authorities assume that this expenditure will be grant-financed. Nevertheless, should IDP-related grants not be forthcoming, the authorities will implement contingency measures to contain the deficit to 4.6 percent of GDP. Staff noted that this may involve cutting non-IDP development spending and other nonessential outlays. And, staff reiterated that the program imposes no limits on the amount of IDP-related spending that could be financed by nonbudgetary grants. 
30. The authorities' decision to delay by one year the phasing out of electricity tariff differential subsidies implies significant additional expenditures. Tariff differential subsidies of Rs. 55 billion ( 0.4 percent of GDP), which are not included in the approved budget, will be required in the current fiscal year. Moreover, the amount of electricity sector debt to be assumed by the government will be somewhat higher than budgeted (Rs. 277 billion as compared to Rs. 216 billion), resulting in additional interest payments of Rs. 10 billion. The government is in the process of hiring an internationally-renowned firm to audit the debt to be assumed by the budget. This audit, to be finalized by end-September, is expected to help address governance concerns. To compensate for the higher-than-budgeted subsidies on account of delayed electricity tariff increases, higher-than-budgeted interest payments for electricity sector debt, and lower revenues from the petroleum levy, the authorities decided to reduce nonpriority development spending, while protecting social safety net, education, and health spending.

31. Staff questioned the prominence of sectoral subsidies in the budget. The authorities emphasized the importance of supporting socially-sensitive sectors and export promotion in the current global economic environment. Staff welcomed the reduction in fertilizer subsidies from 0.3 percent of GDP in 2008/09 to 0.1 percent in 2009/10, but noted that budgetary resources would continue to be used for poorly targeted subsidy schemes, including for wheat and sugar, which undermined a level playing field for investors. Staff also raised concerns about the budgetary contribution for the envisaged Export Investment Development Fund.

\section{Staff discussed measures to ensure adequate central government control over} provincial spending. To this effect, the authorities agreed to limit the use of the SBP overdraft by provinces to six weeks of their wage bills. The federal government, in consultation with provincial governments, will introduce a respective binding Ways and Means ceiling effective August 1, 2009. Moreover, the provinces will reach an agreement with the Ministry of Finance and the SBP by end-September 2009 on a schedule for reduction of the outstanding debt stock resulting from provincial overdrafts (SSMEFP $₫ 13$ ).

\section{Monetary and Exchange Rate Policies}

33. Staff argued that the policy interest rate should remain on hold until core inflation shows a further significant decline; an appropriately tight monetary stance would also help contain price pressures from the more expansionary fiscal policy. The authorities argued that the recent declines in headline and core inflation justified a further reduction in the policy rate. The staff urged caution, as core inflation had not come down as rapidly as anticipated, fiscal revenue was still under pressure, and the timing of external disbursements was highly uncertain. Staff also emphasized the need to combat inflationary pressures from higher fuel and energy prices and increased public sector wages. The authorities agreed to keep the factors influencing interest rate policy under review, expecting that economic and financial developments would allow for easing interest rates soon. 
34. Public sector domestic borrowing requirements will remain high, despite the Tokyo-related disbursements covering the increased budget financing needs. The overall public sector borrowing requirement is expected to increase due to (i) higher projected credit to public sector enterprises for resolution of the "circular" debt and (ii) an increase in credit for commodity operations (e.g., wheat procurement underwritten by the government). To ensure that public sector borrowing does not further constrain lending to the private sector, the SBP and the Ministry of Finance are working on increasing the attractiveness of government bonds as a saving/investment instrument for individuals and nonbank institutional investors.

\section{Meanwhile, the SBP has laid the basis for reforming its monetary policy}

framework. To that end, an explicit corridor for overnight money market rate will be introduced in mid-August 2009 to strengthen the role of the interest rate as a policy instrument. The width of the interest rate corridor will initially be 300 basis points with a view to narrowing it to 200 basis points at a later stage. Key additional changes in support of the corridor include (i) the lengthening of the reserve maintenance period from one to two weeks; (ii) fully-lagged determination of the reserve requirement; and (iii) greater standardization of open market operations. The authorities prefer to review the policy rate quarterly, but are aware that the interest rate corridor may require more frequent policy rate adjustments. Significant enhancements of the monetary policy framework by strengthening the independence of the SBP, are also envisaged under the proposed amendments to the SBP Act (to be presented to parliament by end-September 2009-SSMEFP $\{33$ ), including the creation of an independent Monetary Policy Committee.

36. With increased reliance on interest rates for monetary management, exchange rate policy will be geared more decisively toward the attainment of the reserve accumulation targets. The authorities recognized that exchange rate policy would need to remain flexible to reduce external vulnerabilities, ensure external sustainability, and foster competitiveness. The SBP will pursue a flexible exchange rate policy and continue to phase out the provision of foreign exchange for oil imports. In this regard, the SBP has issued a circular announcing that it will cease providing foreign exchange for import of diesel and other refined petroleum products effective August 1, 2009.

\section{Financial Sector Issues}

37. NPLs have increased but bank capital and liquidity remain adequate. The SBP recognized that deteriorating asset quality (with NPLs likely to have increased further in the second quarter of 2009) may transmit into the solvency indicators, but it considered that this risk was being addressed through a strict enforcement of accelerated provisioning requirements (25 percent for all loans overdue by 90 days). ${ }^{9}$ The SBP expects the NPLs to

\footnotetext{
${ }^{9}$ Of the total NPLs, which reached Rs. 379 billion at end-March, Rs. 263 billion has been fully provisioned.
} 
continue increasing for another quarter but, based on actions taken by the commercial banks to restrain consumer lending and with the financial situation of the textile sector bottoming out, it remains confident that asset quality indicators will gradually improve later in 2009/10, especially if the credit to private sector recovers. Nevertheless, several banks will need to restructure their large exposures. Moreover, a bankruptcy law will be submitted to parliament in August 2009 to help rehabilitate weak but viable companies and liquidate insolvent ones.

38. The SBP has continued to enhance the regulatory framework. Preparations of legislative amendments to strengthen the effectiveness of SBP enforcement in the area of banking supervisions are under way. Draft amendments have already been discussed by the government and will be sent to parliament before end-August (SSMEFP 931 ). In order to create more breathing room for banks, the SBP has revised downward the targeted increases in minimum capital requirements (to be met gradually), but has maintained unchanged the minimum capital adequacy ratios to ensure the financial strength of banks.

\section{Augmentation}

39. The authorities have requested augmentation of access under the $S B A$ of $S D R 2.0674$ billion (200 percent of quota) to meet the country's increased balance of payments needs. If approved, the requested augmentation would increase access under the SBA from 500 to 700 percent of quota, allowing for a further strengthening of the level of international reserves. Increased access is justified as insurance against increased risks stemming from the deeper and more sustained deterioration in global economic conditions than initially expected. In this context, the augmentation will provide a strengthen buffer against downside risks associated with (i) a further and possibly sharper slowdown in exports; (ii) a drop in remittances; and (iii) continued constraints on private external financing. Additional access will also allow the authorities to better manage the pressures on reserves from inconsistencies in timing between priority spending needs and donor disbursements.

40. Augmentation is proposed to be phased in within the duration of the SBA as extended through end-2010. Because of the delay in completing the second review, the augmentation must be combined with (i) a rephasing of purchases; and (ii) the extension of the arrangement and a change in the projected dates of availability to ensure a realistic timeline for the disbursements of Fund resources.

\section{Use of Fund resources for budget financing}

41. Given that the disbursements of Tokyo-related donor support in 2009/10 are likely to be back-loaded, a portion of Fund resources will be used for budget financing. This would allow the government to meet urgent spending needs early in 2009/10, including the provision of assistance to IDPs, without having to rely on additional domestic financing which could undermine economic stabilization. It would also help resolve the potential 
balance-of-payments problem by avoiding undue pressures on the reserve position and foreign exchange market.

42. To this effect, about \$1.4 billion (0.8 percent of GDP) of Fund resources will be made available, on a temporary basis, for budget financing during 2009/10. This amount broadly corresponds to social sector and IDP-related priority spending financed from additional donor funds pledged in Tokyo. This amount is also within Pakistan's capacity to repay the Fund, assuming that the targeted adjustment is achieved (see $\uparrow 43$ ).

- $\quad$ The designated part of Fund resources will be credited directly to the Ministry of Finance's dedicated account at the SBP in three installments at the time of Fund disbursements projected for August 2009-March 2010. The budget will draw on this account to finance its priority needs until Tokyo-related pledges are disbursed. The budget will be responsible for servicing any outstanding Fund credit used for budgetary purposes through the SBP, which remains the fiscal agent.

- $\quad$ The budget will replenish the dedicated account to the amount borrowed from the Fund as Tokyo-related pledges are disbursed (expected by end-2009/10), at which time the SBP will assume the related liability to the Fund and debt servicing.

- If the Tokyo-related disbursements fall short of the projected $\$ 2$ billion in 2009/10, part of this shortfall (up to $\$ 1.4$ billion) at year's end would be covered by Fund resources. In this event, the budget will replenish the dedicated account as soon as the delayed disbursements are received. In any event, however, Fund resources would not be used to cover shortfalls in the disbursement of pledges from multilateral donors.

\section{Capacity to repay the Fund}

43. The proposed augmentation is within Pakistan's capacity to repay the Fund, provided the program is successfully implemented. With the augmentation, Pakistan's GRA credit outstanding would peak at nearly 72 percent of gross international reserves, equivalent to 5.9 percent of GDP, by 2010/11 (compared with 65 percent of gross international reserves in 2009/10 at the time the SBA was approved), while debt service payments would peak at about 35.9 percent of end-period gross reserves and 15.3 percent of exports of goods and services in 2013/14. These metrics are high relative to other exceptional cases, and indicate substantial risks to the capacity to repay the Fund. However, the authorities' past record of servicing Fund obligations has been very good.

44. Given the continued balance-of-payments pressures and the strength of program commitments, Pakistan continues to meet the four criteria for exceptional access (Box 3 ).

\section{The Medium-term Outlook And Debt Sustainability}

45. The medium-term economic outlook is fragile. A slower global recovery, higher commodity prices, and political instability, as well as existing constraints on energy and 


\section{Box 3. Exceptional Access Criteria}

Criterion 1: Experiencing or potential to experience exceptional balance of payments pressures on the current or capital accounts. Pakistan continues to experience large balance of payments pressures. The rebound in oil prices has rekindled pressures on the current account, and risks of lower exports and remittances could further increase the current account deficit. In addition, capital inflows - already much lower than originally envisaged - could decline further on account of global de-leveraging and loss of investor confidence due to domestic political instability. These increased pressures result in a need for Fund financing that cannot be met within the normal limits. Reserves are expected to recover by the end of the program period to levels considered adequate by traditional metrics, but will remain well below pre-crisis levels and those of comparator countries.

Criterion 2: Sustainable public debt in the medium term. Pakistan's public debt (domestic and external, excluding obligations to the IMF), is projected to remain at around 57 percent of GDP over the medium term. Public debt, including obligations of the IMF is projected to peak at 63.5 percent of GDP in 2010/11, and decline to 57.8 percent of GDP in 2013/14. The significant increase in the public debt-to-GDP ratio compared with that at the time of the SBA approval reflects mainly the government decision to assume the electricity sector debt and the increased borrowing needs of the budget to deal with the impact of the global economic crisis on domestic economy, as well as increased security and social spending. There is a high probability that Pakistan's public debt will remain sustainable in the medium term; the reforms envisaged under the program and the projected fiscal consolidation will enable a gradual decline in the public debt-to-GDP ratio. The external debt would increase from 29.9 percent of GDP in 2008/09 to 35.5 percent in 2010/11, before declining gradually to 32.5 percent of GDP by $2013 / 14$. Domestic interest payments are projected to decline, while the external debt service burden is projected to rise but remain manageable.

Criterion 3: Access to private capital markets. Until recently, Pakistan had been able to access international financial markets by issuing Eurobonds, Global Depository Receipts, and exchangeable bonds, as well as through nonresidents' portfolio investment in domestic securities. Pakistan has maintained a good record in servicing its external private debt despite difficult economic conditions. It is expected that the country can regain access to international capital markets and see a pickup in FDI within two to three years, provided that the adjustment effort is successfully implemented, financial market conditions continue to normalize, and cross-border private capital flows recover to pre-crisis levels.

Criterion 4: Strong policy reform program. Program implementation has been broadly satisfactory. The staff continues to believe that Pakistan has sufficient institutional capacity to deliver the required adjustment, as evidenced by the successful implementation of Fund-supported programs during 2000/01-2004/05, and performance under the current SBA. Staff also believes that Pakistan has the political capacity to deliver the required adjustment. However, while there are reasonable prospects for success if the proposed policies are implemented, the risks to the program remain very high, as implementation can be affected by the difficult political, security, and economic conditions.

infrastructure pose significant risks to the outlook. The global crisis, which erupted with full force after the adoption of the program, has significantly affected Pakistan's medium-term economic prospects. Growth is likely to be lower than originally envisaged and private 
capital inflows will take longer to recover. As a result, stronger policies will be needed to support competitiveness and the medium-term fiscal adjustment effort. Also, higher domestic borrowing and/or foreign debt-creating inflows (including Tokyo-related) will be needed to smooth the impact of the adjustment on consumers and taxpayers.

46. The external debt sustainability analysis shows that the debt stock would remain moderate and external debt service manageable. The external debt stock would increase from 29.9 percent of GDP in 2008/09 to 35.5 percent in 2010/11, before declining gradually to 32.5 percent of GDP by $2013 / 14$. The debt service as a ratio of exports of goods and receipts is projected to increase from below 15 percent to 20 percent during the same period, reflecting in part, repayments of Fund credit. This outlook, however, is subject to serious downside risks, including a possibly higher noninterest current account deficit, slower growth, larger depreciation, higher interest rates, and lower FDI flows. Combined shocks to growth, current account, and depreciation could vault the end-period debt stock to around 49 percent of GDP, a level significantly higher than in the baseline scenario.

47. Since the conclusion of the last Article IV consultation in April 2009, public sector debt sustainability prospects have been affected by the assumption of electricity sector debt by the budget and the higher projected deficit. Total public sector debt (excluding obligations to the IMF) relative to GDP is now expected to increase from 56 percent in $2008 / 09$ to 56.8 percent in $2009 / 10$. This reflects primarily the upward revision of the 2009/10 fiscal deficit target to 4.6 percent of GDP to allow for the spending of the additional donor financing pledged in Tokyo and the assumption by the budget of electricity sector debt of 1.9 percent of GDP. While the last public sector DSA projected a gradual decline in this ratio, it is now projected to stay virtually constant around 57 percent through $2012 / 13$, with a marginal decline (to 56.3 percent) starting only in 2013/14. This reflects a projected increase in foreign borrowing by 7.3 percentage points of GDP between 2009/10 and 2013/14, while domestic debt is projected to decline at a similar pace. Public debt (including obligations to the Fund) will reach a peak of 63.5 percent of GDP in 2010/11 and decline to 57.8 percent of GDP in 2013/14. While there is a high probability that the debt will remain sustainable assuming the program is fully implemented, the outlook is subject to significant downside risks. The standard bound tests show that all shocks (primary balance shock, interest rate shock, growth shock, and real exchange rate shock) lead to an increase in the debt-to-GDP ratio by several percentage points. The effect of a real exchange rate shock is particularly pronounced, reflecting the rising weight of external debt in total public debt. This implies that the authorities will need to stand ready to take additional fiscal measures, as needed.

\section{Staff Appraisal}

48. Despite shortcomings in policy implementation, Pakistan's economy has continued to stabilize. Reforms in the financial sector and the foreign exchange market have been progressing broadly as envisaged, and steps have been taken to strengthen the social safety net. Revenue mobilization efforts, however, have proven inadequate, as tax policy and tax 
administration reforms have been delayed. Also, major expenditure overruns in the last quarter of 2008/09 revealed significant weakness in control over provincial spending. Moreover, the authorities have not been decisive in addressing long-standing problems in the energy sector. Together with the global crisis, energy problems have affected adversely Pakistan's growth potential and burdened public finances.

49. Tax reforms are needed to make the economy less vulnerable and allow the steady flow of revenue needed to reduce poverty and develop basic infrastructure. Increasing tax revenue is crucial for reducing macroeconomic vulnerability and providing lasting fiscal space for poverty-reducing spending. The low revenue effort constrains the government's ability to deal with the pressing needs of the population, which are now compounded by the large number of IDPs. In this regard, the authorities need to move decisively with the VAT preparations and adhere strictly to the timetable for introducing the VAT on July 1, 2010. Of equal importance is the renewed commitment to tax administration reform.

50. The delay in implementing much needed reforms in the energy sector drains the public finances and constrains the country's medium-term growth prospects. This entails significant budgetary costs (in the form of subsidies and debt service payments) and undermines macroeconomic stabilization. The authorities need to proceed swiftly with the envisaged tariff increases and the implementation of the new automatic tariff adjustment mechanism to de-politicize the tariff adjustment process. Further delays or backtracking will result in the crowding out of other needed expenditure, and inadequate investment to increase generation and distribution capacity.

51. The macroeconomic outlook for 2009/10 remains difficult, and the external position is subject to considerable downside risks. Pakistan's main economic challenges in 2009/10 will be (i) consolidating macroeconomic stability and maintaining the momentum of supporting reforms, including in the energy sector; (ii) dealing with the impact of the global crisis on exports and economic activity; and (iii) mobilizing political support for tax policy and administration reforms. To achieve the program objectives, the authorities will need to focus on mobilizing donor support, pressing ahead with fiscal consolidation, and tax, electricity sector, and financial sector reforms.

52. The additional donor support pledged in the Tokyo conference and the augmentation of access under the $S B A$ will help meet these challenges, but such financing should be regarded as temporary and used as a bridge until a much stronger medium-term revenue effort bears fruit. Mobilizing higher revenue to reduce the public debt-to-GDP ratio should be a key policy objective. A credible tax reform is needed to ensure fiscal sustainability and to create fiscal space for expanding the social safety net, and increasing investment in human and physical capital.

53. The 2009/10 budget has been constructed with a view to providing adequate space for priority spending, but its implementation should be monitored closely. In particular, the 
authorities should ensure that the redistributive function of the budget is consistent with the government's social policy objectives, with spending on IDPs, support for poor households, and other well-targeted social spending taking precedence over transfers to politically connected groups. Also, development spending must be properly timed to ensure adequate resources for IDPs, regardless of the availability of the external grants.

54. Monetary policy needs to be vigilant to prevent a resurgence of inflation. The relaxation of the fiscal policy stance, electricity tariff increases, and the rebound in oil prices will add to inflationary pressures. Moreover, the present round of wage increases in the public sector, if not managed properly, may trigger an economy-wide realignment of wages, with attendant effects on inflation and competitiveness.

55. Exchange rate policy should aim at strengthening competitiveness and further strengthening the international reserves position. In this regard, the accelerated phasing out of the foreign exchange provision for oil imports payments is a key step in the right direction.

\section{Accelerating reforms to strengthen the independence of the SBP and pressing} forward with the legislative amendments to increase SBP's supervisory powers is of great importance. Theses reforms are critical for enhancing the monetary policy framework and maintaining the stability of the banking system in the present difficult environment.

\section{Despite the difficulties in implementing program commitments, the authorities have} made significant efforts to keep the program on track. Progress made toward reducing inflation and strengthening the external position, the measures to improve the social safety net, and the adoption of a budget for 2009/10 that provides adequate basis for further macroeconomic stabilization and much needed social spending, constitute appreciable achievements, particularly taking into account the security developments, the global economic recession, and the difficult domestic political environment. The nonobservance of the end-June performance criterion on the fiscal deficit reflects to a large extent temporary pressures on account of unforeseen security- and IDP-related expenditure needs in the last quarter of 2008/09 and excessive provincial spending. A waiver is justified given the temporary nature of these unforeseen expenditures and the measures to control the use of overdrafts by provinces. The recent agreement with World Bank and ADB staffs on the electricity sector reform also signals a desire to address the deep-seated problems in that sector. With these considerations in mind and based on the strength of the updated commitments expressed by the authorities in the attached SSMEFP (including the corrective actions aiming to enhance the implementation of the structural reforms under the program), staff supports the requested waivers of nonobservance for the two missed structural performance criteria and the end-June quantitative performance criterion on the fiscal deficit, waivers of applicability for other end-June quantitative performance criteria, the rephasing of purchases and the extension of the arrangement through end-2010, and recommends the completion of the second review and the approval of the augmentation of access by 200 percent of quota. 
Figure 1. Pakistan: Selected Economic Indicators

(June 2008-End June 2009, unless otherwise indicated)
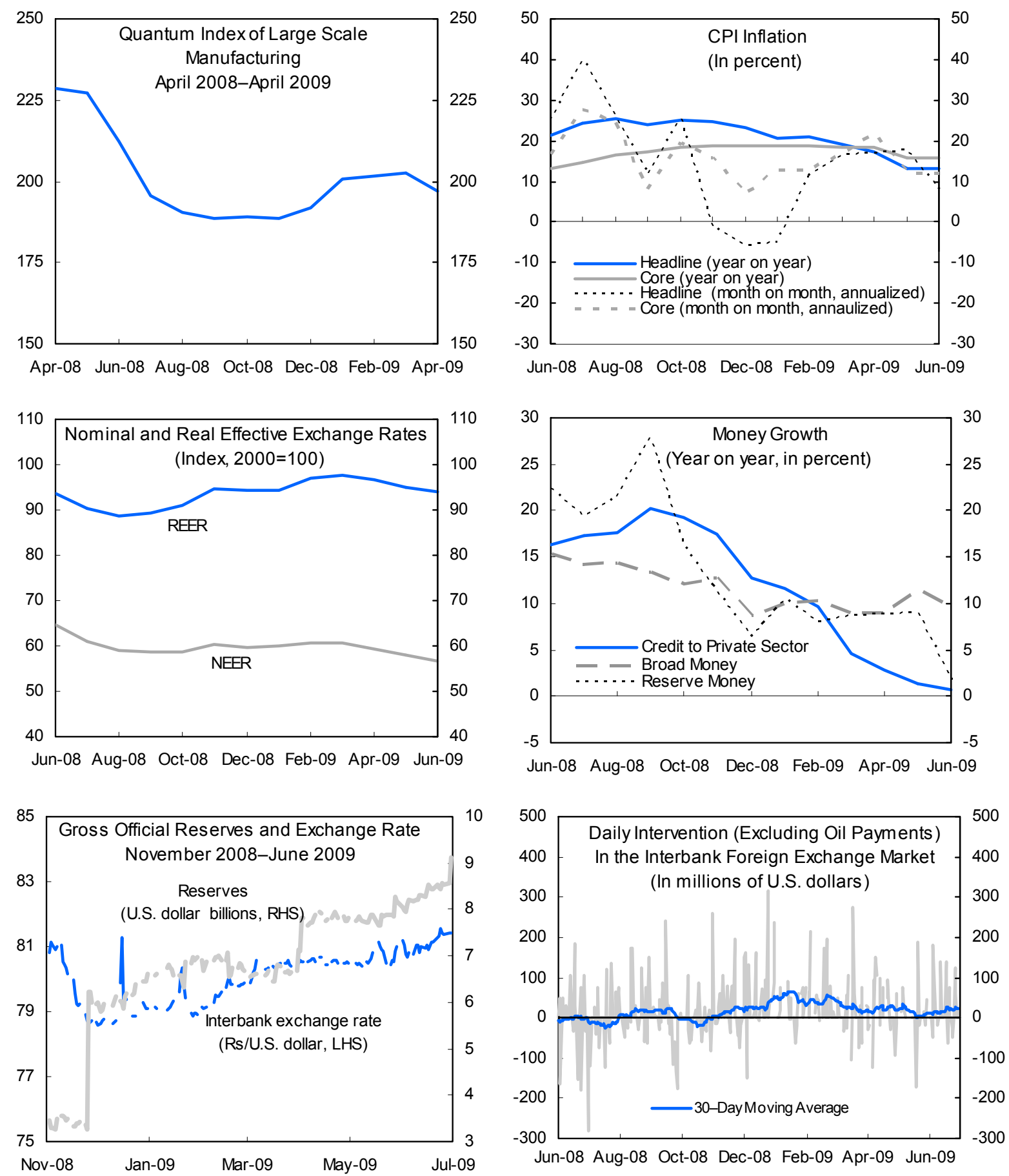

Source: Pakistani authorites. 
Figure 2. Pakistan: Financial Market Indicators
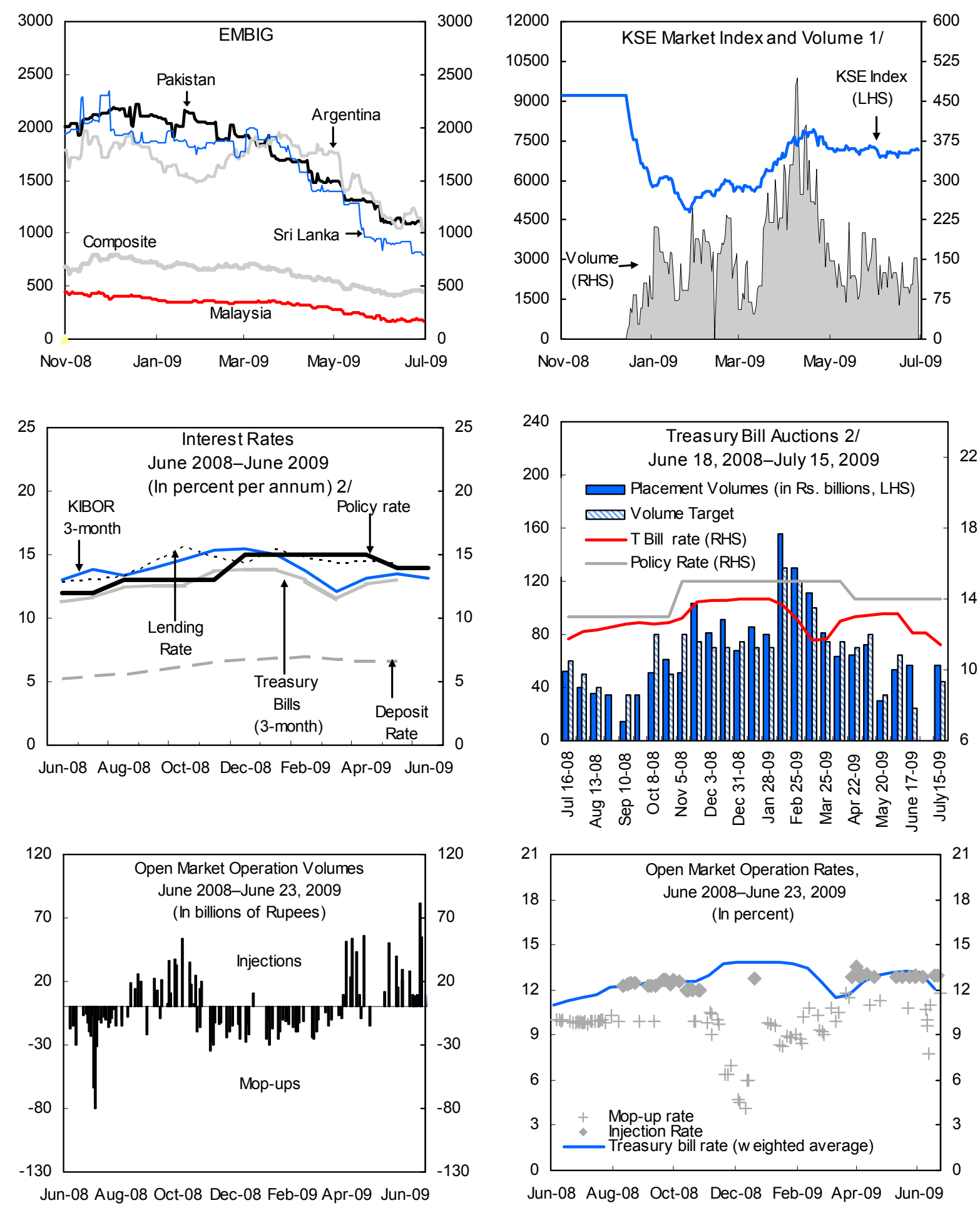

Sources: Pakistani authorities; and Bloomberg.

$1 /$ Volume in millions of shares.

2/ Placement volumes are for all maturities and Treasury bill rate is a weighted average. 
Figure 3. Pakistan: Real and External Sectors, 2005/06-2009/10
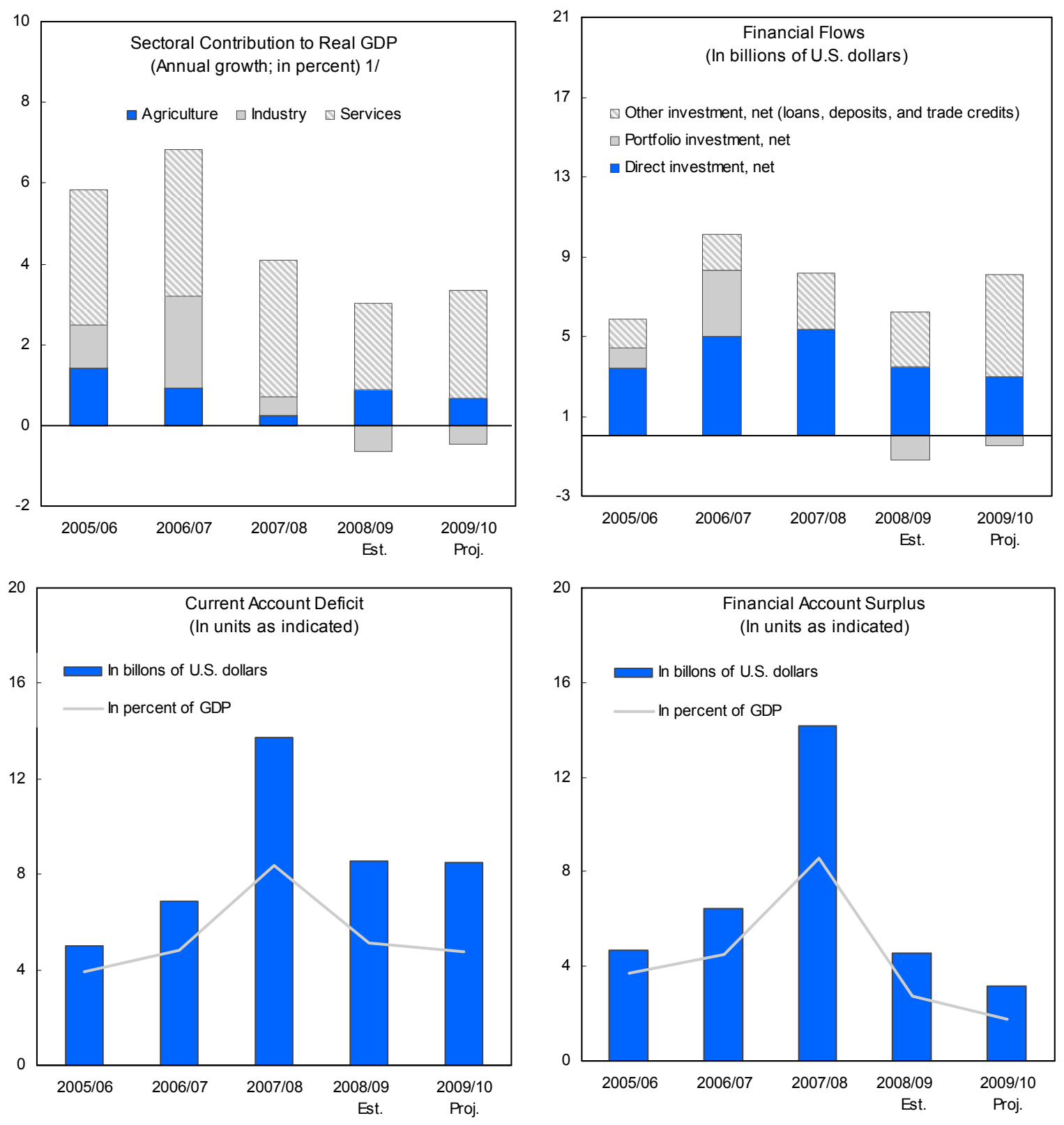

Sources: Pakistan authorities and Fund staff projections.

1/ At factor cost. 
Figure 4. Pakistan: Fiscal Policy Indicators, 2007/08-2009/10
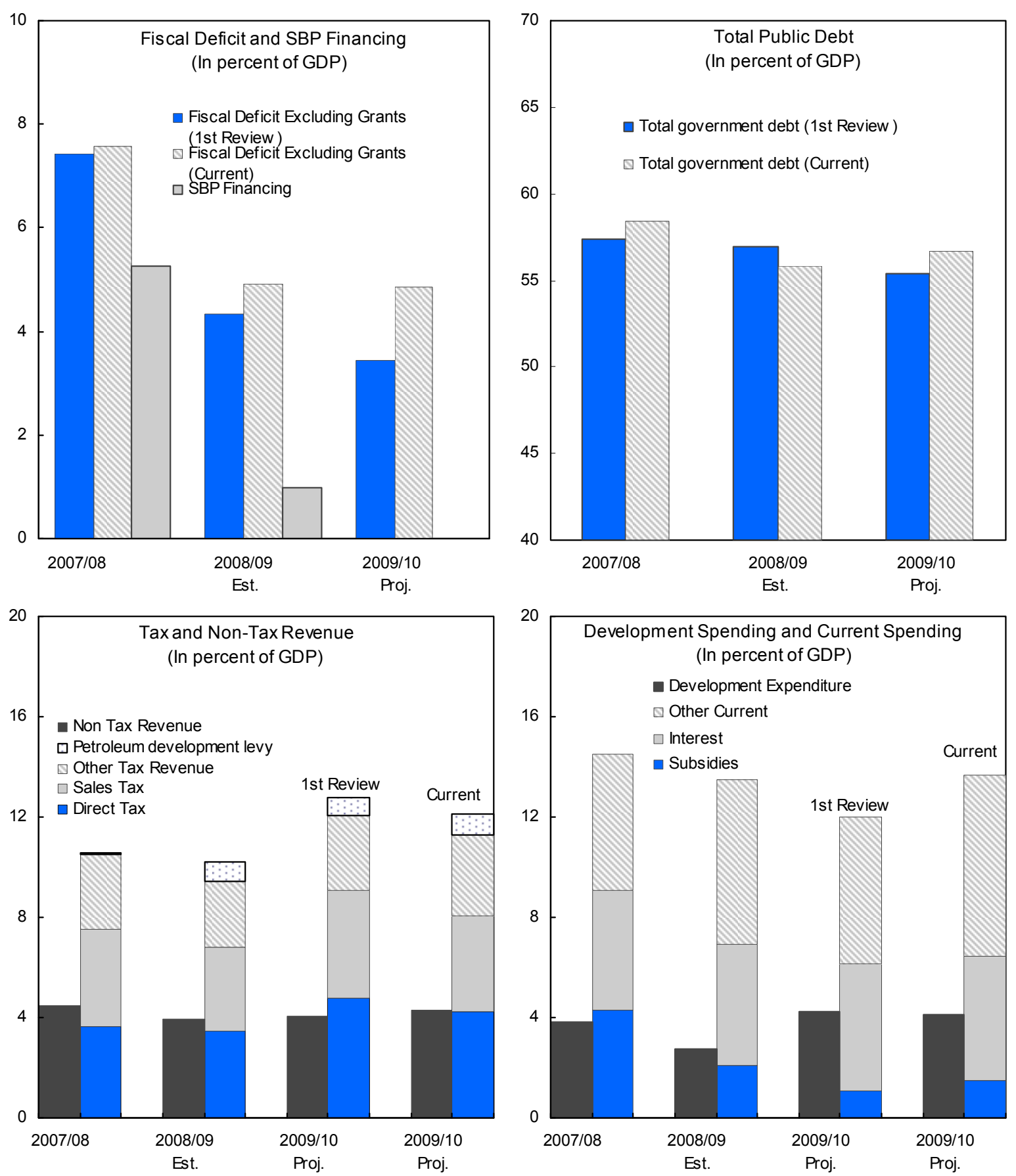

Sources: Pakistan authorities and Fund staff projections. 
Table 1. Pakistan: Selected Economic Indicators, 2007/08-2009/10 1/

(Population: 160.9 million (2007/08))

(Per capita GDP: U.S.\$1,042 (2007/08))

(Poverty rate: 23.9 percent $(2004 / 05))$

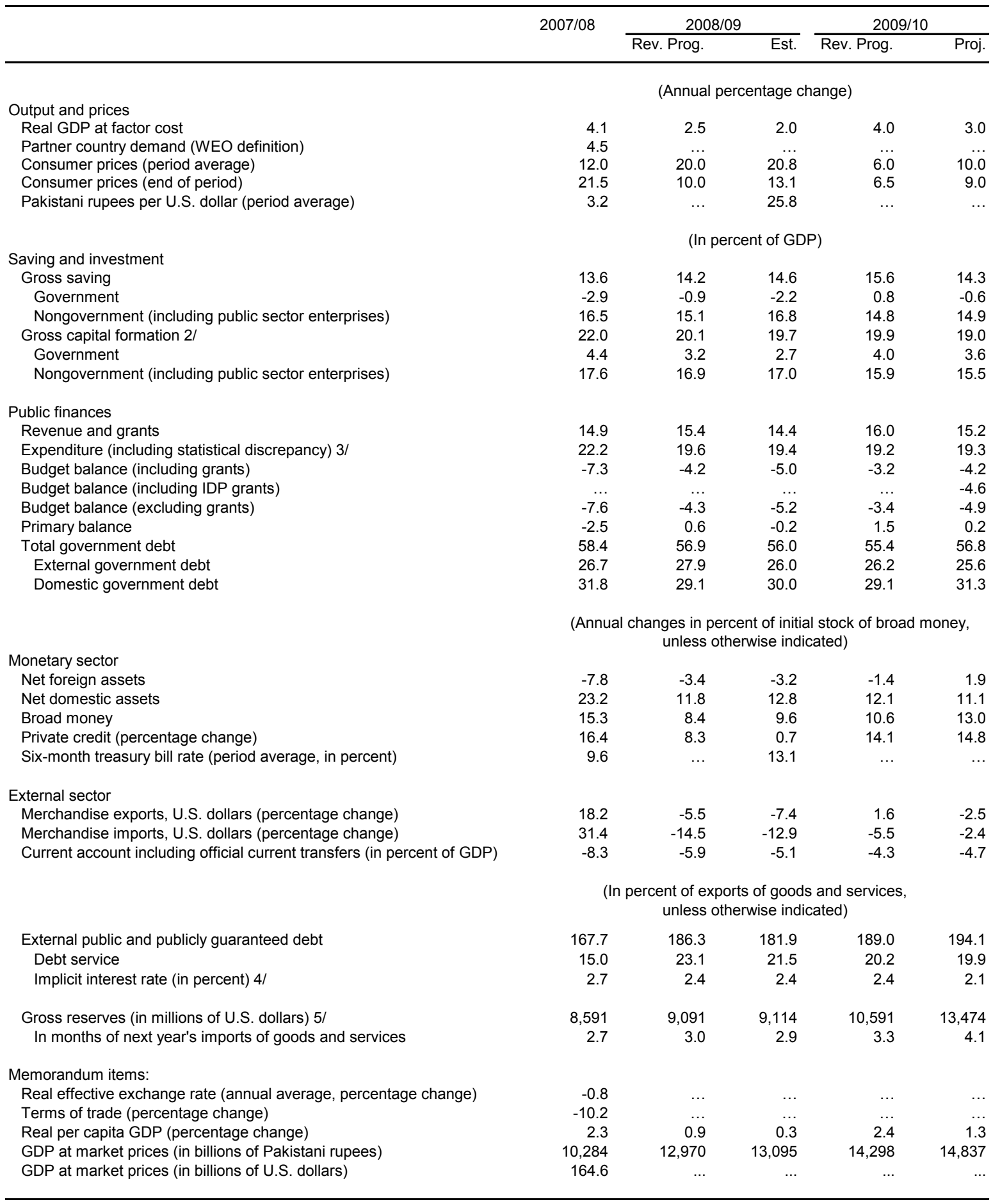

Sources: Pakistani authorities; and Fund staff estimates and projections.

1/ Fiscal year ends June 30 .

2/ Including changes in inventories. Investment data recorded by the Pakistan Federal Bureau of Statistics are said to underreport true activity. 3 / Expenditure on social assistance in 2008/09 is budgeted at 0.5 percent of GDP. The program targets an additional 0.3-0.5 percent of GDP.

4/ Calculated as interest payments in percent of the end-of-period debt stock of the previous year.

5/ Excluding gold and foreign deposits of commercial banks held with the State Bank of Pakistan. 
Table 2. Pakistan: Balance of Payments, 2007/08-2009/10

(In millions of U.S. dollars; unless otherwise indicated)

\begin{tabular}{|c|c|c|c|c|c|c|c|c|c|}
\hline & \multirow[t]{3}{*}{$2007 / 08$} & \multicolumn{2}{|c|}{$2008 / 09$} & \multicolumn{6}{|c|}{$2009 / 10$} \\
\hline & & \multicolumn{2}{|c|}{ Year } & Jul-Sep & Oct-Dec & Jan-Mar & Apr-Jun & \multicolumn{2}{|c|}{ Year } \\
\hline & & Rev. Prog. & $\overline{\text { Est. }}$ & \multicolumn{4}{|c|}{ Projection } & Rev. Prog. & Proj. \\
\hline Current account & $-13,735$ & $-9,643$ & $-8,551$ & $-2,192$ & $-2,003$ & $-2,361$ & $-1,931$ & $-7,407$ & $-8,487$ \\
\hline Balance on goods & $-15,045$ & $-11,274$ & $-11,965$ & $-3,292$ & $-2,689$ & $-3,040$ & $-2,671$ & $-9,316$ & $-11,692$ \\
\hline Exports, f.o.b. & 20,427 & 19,015 & 18,915 & 4,241 & 4,241 & 4,795 & 5,163 & 19,311 & 18,441 \\
\hline Imports, f.o.b. & $-35,472$ & $-30,290$ & $-30,880$ & $-7,533$ & $-6,931$ & $-7,835$ & $-7,835$ & $-28,627$ & $-30,133$ \\
\hline Services (net) & $-6,257$ & $-3,872$ & $-3,180$ & -311 & -610 & -820 & -713 & $-3,526$ & $-2,454$ \\
\hline Services: credit & 3,577 & 3,805 & 4,017 & 1,300 & 1,001 & 1,001 & 1,248 & 3,873 & 4,550 \\
\hline Services: debit & $-9,834$ & $-7,677$ & $-7,197$ & $-1,611$ & $-1,611$ & $-1,821$ & $-1,961$ & $-7,399$ & $-7,005$ \\
\hline Income (net) & $-3,909$ & $-4,234$ & $-4,471$ & $-1,000$ & $-1,080$ & $-1,065$ & $-1,187$ & $-4,062$ & $-4,333$ \\
\hline Income: credit & 1,613 & 1,200 & 898 & 209 & 209 & 236 & 254 & 1,218 & 909 \\
\hline Income: debit & $-5,522$ & $-5,434$ & $-5,369$ & $-1,209$ & $-1,289$ & $-1,301$ & $-1,442$ & $-5,279$ & $-5,241$ \\
\hline Of which: interest payments & $-2,161$ & $-2,158$ & $-1,913$ & -429 & -509 & -419 & -492 & $-1,873$ & $-1,850$ \\
\hline Of which: income on direct investment & $-3,361$ & $-3,276$ & $-3,456$ & -780 & -780 & -882 & -950 & $-3,407$ & $-3,391$ \\
\hline Balance on goods, services, and income & $-25,211$ & $-19,380$ & $-19,616$ & $-4,603$ & $-4,379$ & $-4,925$ & $-4,572$ & $-16,904$ & $-18,479$ \\
\hline Current transfers (net) & 11,476 & 9,737 & 11,065 & 2,411 & 2,376 & 2,564 & 2,641 & 9,497 & 9,992 \\
\hline Current transfers: credit, of which: & 11,618 & 9,875 & 11,174 & 2,424 & 2,389 & 2,580 & 2,657 & 9,603 & 10,050 \\
\hline Official & 484 & 175 & 158 & 181 & 146 & 97 & 40 & 103 & 464 \\
\hline Workers' remittances & 6,451 & 7,200 & 7,751 & 1,608 & 1,608 & 1,675 & 1,809 & 7,000 & 6,700 \\
\hline Other private transfers & 4,683 & 2,500 & 3,265 & 635 & 635 & 808 & 808 & 2,500 & 2,886 \\
\hline Current transfers: debit & -142 & -138 & -109 & -13 & -13 & -16 & -16 & -106 & -58 \\
\hline Capital account & 121 & 121 & 164 & 316 & 324 & 249 & 75 & 284 & 964 \\
\hline Capital transfers: credit & 128 & 127 & 166 & 318 & 326 & 252 & 71 & 295 & 967 \\
\hline Of which: official capital grants & 111 & 108 & 141 & 314 & 322 & 248 & 68 & 271 & 952 \\
\hline Of which: Tokyo pledge & $\ldots$ & $\ldots$ & $\ldots$ & 142 & 200 & 200 & 20 & $\ldots$ & 562 \\
\hline Capital transfers: debit & -7 & -6 & -2 & -2 & -2 & -3 & 4 & -11 & -3 \\
\hline Financial account & 8,182 & 5,459 & 4,996 & 904 & 1,659 & 1,690 & 3,371 & 6,628 & 7,625 \\
\hline Direct investment abroad & -75 & -34 & -23 & -10 & -15 & -20 & 15 & -80 & -30 \\
\hline Direct investment in Pakistan & 5,410 & 3,800 & 3,476 & 500 & 700 & 700 & 1,080 & 4,066 & 2,980 \\
\hline Of which: privatization receipts & 133 & 0 & 0 & 0 & 133 & 133 & 0 & 266 & 266 \\
\hline Portfolio investment (net), of which: & 36 & -957 & $-1,218$ & -22 & 0 & -600 & 138 & -322 & -484 \\
\hline Eurobond/GDR & -25 & -525 & -525 & -22 & 0 & -600 & 0 & -522 & -622 \\
\hline Other investment assets & 32 & -445 & 836 & -100 & -100 & -104 & 20 & -95 & -284 \\
\hline General government & 5 & 5 & 7 & 0 & 0 & -4 & -4 & 5 & -8 \\
\hline Banks & 525 & -350 & 327 & -50 & -50 & -50 & -50 & 100 & -200 \\
\hline Other sectors & -498 & -100 & 502 & -50 & -50 & -50 & 74 & -200 & -76 \\
\hline Other investment liabilities & 2,779 & 3,095 & 1,925 & 536 & 1,074 & 1,714 & 2,118 & 3,059 & 5,443 \\
\hline Monetary authorities & 490 & 0 & 0 & 0 & 1,267 & 0 & 0 & 0 & 1,267 \\
\hline General government, of which: & 1,808 & 2,773 & 1,976 & 491 & 63 & 758 & 2,214 & 1,930 & 3,526 \\
\hline Disbursements & 3,078 & 5,158 & 4,313 & 815 & 955 & 1,158 & 2,480 & 3,287 & 5,408 \\
\hline Of which: Tokyo pledge & & & & 200 & 635 & 685 & 408 & $\ldots$ & 1,928 \\
\hline Amortization & $-1,249$ & $-2,385$ & $-2,337$ & -324 & -892 & -400 & -266 & $-1,957$ & $-1,882$ \\
\hline Banks & 66 & -212 & -587 & 22 & 22 & 28 & 28 & 300 & 100 \\
\hline Other sectors & 415 & 534 & 536 & 23 & -278 & 928 & -124 & 829 & 550 \\
\hline Net errors and omissions & -356 & -404 & 99 & 0 & 0 & 0 & 0 & 0 & 0 \\
\hline Reserves and related items & 5,788 & 4,467 & 3,292 & 972 & 20 & 422 & $-1,516$ & 495 & -102 \\
\hline Reserve assets, of which: & 5,961 & -188 & -457 & -150 & $-1,050$ & -700 & $-2,560$ & $-1,850$ & $-4,460$ \\
\hline Foreign exchange (State Bank of Pakistan) & 5,711 & -500 & -523 & -100 & $-1,000$ & -600 & $-2,660$ & $-1,500$ & $-4,360$ \\
\hline Foreign exchange (deposit money banks) & 250 & 312 & 66 & -50 & -50 & -100 & 100 & -350 & -100 \\
\hline Use of Fund credit and loans & -173 & 4,555 & 3,749 & 1,122 & 1,070 & 1,122 & 1,044 & 2,345 & 4,358 \\
\hline Exceptional financing & 0 & 100 & 0 & 0 & 0 & 0 & 0 & 0 & 0 \\
\hline \multicolumn{10}{|l|}{ Memorandum items: } \\
\hline Current account (in percent of GDP) & -8.3 & -5.9 & -5.1 & -1.2 & -1.1 & -1.3 & -1.1 & -4.3 & -4.7 \\
\hline Current account (in percent of GDP; excluding fuel imports) & -2.0 & -0.2 & 0.8 & $\ldots$ & $\ldots$ & $\ldots$ & $\ldots$ & -0.3 & 1.0 \\
\hline Exports f.o.b. (growth rate, in percent) & 18.2 & -5.5 & -7.4 & $\ldots$ & $\ldots$ & $\ldots$ & $\ldots$ & 1.6 & -2.5 \\
\hline Imports f.o.b. (growth rate, in percent) & 31.4 & -14.5 & -12.9 & $\ldots$ & $\ldots$ & $\ldots$ & $\ldots$ & -5.5 & -2.4 \\
\hline Imports f.o.b. (growth rate, in percent, excluding oil) & 26.8 & -15.4 & -15.3 & $\ldots$ & $\ldots$ & $\ldots$ & $\ldots$ & 2.2 & -5.5 \\
\hline Oil imports (in million U.S.\$, cif) & 10,496 & 9,250 & 9,850 & $\ldots$ & $\ldots$ & $\ldots$ & $\ldots$ & 6,957 & 10,333 \\
\hline Non-oil imports & 24,976 & 21,040 & 21,030 & & & & & 21,670 & 19,800 \\
\hline Crude oil price $(\$ / b b l)$ & 87.0 & 80.0 & 68.5 & $\ldots$ & $\ldots$ & $\ldots$ & $\ldots$ & 45.8 & 68.5 \\
\hline Terms of trade (growth rate, in percent) & -10.2 & $\ldots$ & $\ldots$ & $\ldots$ & $\ldots$ & $\ldots$ & $\ldots$ & $\ldots$ & $\ldots$ \\
\hline Workers' remittances and other private transfers & & & & & & & & & \\
\hline (growth rate, in percent) & 10.1 & -12.7 & -1.1 & $\cdots$ & 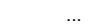 & & & -2.1 & -13.0 \\
\hline External debt (in millions of U.S. dollars) & 44,468 & 51,454 & 49,860 & 52,731 & 54,084 & 57,416 & 57,417 & 55,402 & 57,417 \\
\hline Gross financing needs (in millions of U.S. dollars) 1/ & 15,066 & 12,659 & 11,536 & $\cdots$ & $\ldots$ & & & 10,340 & 11,246 \\
\hline End-period gross official reserves (millions of U.S. dollars) $2 /$ & 8,591 & 9,091 & 9,114 & 9,214 & 10,214 & 10,814 & 13,474 & 10,591 & 13,474 \\
\hline (In months of next year's imports of goods and services) & 2.7 & 3.0 & 2.9 & $\ldots$ & $\ldots$ & $\ldots$ & $\ldots$ & 3.3 & 4.1 \\
\hline (in percent of debt service) & 246.0 & 175.7 & 186.1 & $\ldots$ & $\ldots$ & $\ldots$ & $\ldots$ & 220.4 & 292.3 \\
\hline GDP (in millions of U.S. dollars) & 164,557 & 162,627 & 166,513 & 178,723 & 178,723 & 178,723 & 178,723 & 171,015 & 178,723 \\
\hline
\end{tabular}

Sources: Pakistani authorities; and Fund staff estimates and projections.

1/ Defined as current account deficit, plus amortization on medium- and long-term debt, plus short-term debt at end of previous period.

2/ Excluding foreign currency deposits held with the State Bank of Pakistan (cash reserve requirements) and gold. 
Table 3a. Pakistan: Consolidated Government Budget, 2007/08-2009/10

\begin{tabular}{|c|c|c|c|c|c|c|c|c|c|c|c|}
\hline \multirow{3}{*}{ 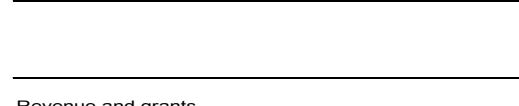 } & \multirow{2}{*}{$\begin{array}{c}2007 / 08 \\
\text { Year }\end{array}$} & \multicolumn{3}{|c|}{$\begin{array}{c}2008 / 09 \\
\text { Year }\end{array}$} & \multirow[b]{2}{*}{ Jul-Sep } & \multicolumn{3}{|r|}{$\begin{array}{l}2009 / 10 \\
\text { Apr-Jun }\end{array}$} & \multirow[b]{2}{*}{ Rev. Prog. } & \multirow[b]{2}{*}{$\frac{\text { Year }}{\text { Budget }}$} & \multirow[b]{2}{*}{ Proj. } \\
\hline & & Prog. & $\begin{array}{l}\text { Year } \\
\text { Rev. Prog. }\end{array}$ & Est. & & \multicolumn{2}{|c|}{$\begin{array}{l}\text { Oct-Dec Jan-Mar } \\
\text { Projection }\end{array}$} & Apr-Jun & & & \\
\hline & 1,530 & 2,018 & 1,996 & 1,888 & 525 & 540 & 526 & 660 & 2,286 & 2,222 & 2,251 \\
\hline $\begin{array}{l}\text { Revenue } \\
\text { Tax reve }\end{array}$ & $\begin{array}{l}1,499 \\
1,086\end{array}$ & $\begin{array}{l}1,995 \\
1,468\end{array}$ & $\begin{array}{l}1,974 \\
1,466\end{array}$ & $\begin{array}{l}1,855 \\
1,340\end{array}$ & $\begin{array}{l}490 \\
334\end{array}$ & $\begin{array}{l}505 \\
396\end{array}$ & $\begin{array}{l}498 \\
375\end{array}$ & $\begin{array}{l}652 \\
479\end{array}$ & $\begin{array}{r}2,255 \\
1707\end{array}$ & $\begin{array}{l}2,156 \\
1,593\end{array}$ & $\begin{array}{l}2,146 \\
1,583\end{array}$ \\
\hline Federal & 1,045 & 1,409 & 1,412 & 1,279 & 316 & 382 & 357 & 458 & 1,642 & 1,523 & 1,513 \\
\hline $\begin{array}{l}\text { FBR revenue } \\
\text { Direct taxes }\end{array}$ & $\begin{array}{r}1,007 \\
388\end{array}$ & $\begin{array}{r}1,360 \\
550\end{array}$ & $\begin{array}{r}1,300 \\
550\end{array}$ & $\begin{array}{r}1,155 \\
450\end{array}$ & $\begin{array}{l}286 \\
116\end{array}$ & $\begin{array}{l}346 \\
147\end{array}$ & $\begin{array}{l}321 \\
123\end{array}$ & & $\begin{array}{l}1,516 \\
639\end{array}$ & $\begin{array}{r}1,380 \\
566\end{array}$ & 1,380 \\
\hline $\begin{array}{l}\text { Direct taxes } \\
\text { Federal excise duty }\end{array}$ & & $\begin{array}{l}530 \\
121\end{array}$ & $\begin{array}{l}530 \\
119\end{array}$ & $\begin{array}{l}450 \\
118\end{array}$ & $\begin{array}{r}116 \\
30\end{array}$ & 147 & $\begin{array}{l}123 \\
42\end{array}$ & $\begin{array}{r}167 \\
56\end{array}$ & 639 & $\begin{array}{l}566 \\
153\end{array}$ & 552 \\
\hline $\begin{array}{l}\text { Federal excise duty } \\
\text { Sales tax }\end{array}$ & $\begin{array}{r}84 \\
385\end{array}$ & $\begin{array}{l}121 \\
516\end{array}$ & $\begin{array}{l}119 \\
499\end{array}$ & $\begin{array}{l}118 \\
444\end{array}$ & $\begin{array}{r}30 \\
108\end{array}$ & $\begin{array}{r}43 \\
120\end{array}$ & $\begin{array}{r}42 \\
118\end{array}$ & $\begin{array}{l}56 \\
154\end{array}$ & $\begin{array}{l}\begin{array}{l}142 \\
573\end{array} \\
573\end{array}$ & $\begin{array}{l}153 \\
499\end{array}$ & $\begin{array}{l}171 \\
500\end{array}$ \\
\hline $\begin{array}{l}\text { Customs duties } \\
\text { Petrolems surcharge }\end{array}$ & 151 & $\begin{array}{l}193 \\
193\end{array}$ & 151 & 143 & 32 & 37 & 39 & $\begin{array}{r}54 \\
49\end{array}$ & 162 & 162 & 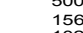 \\
\hline $\begin{array}{l}\text { Petroleum surcharge } \\
\text { Gas surcharge and other }\end{array}$ & $\begin{array}{l}14 \\
23\end{array}$ & $\begin{array}{l}14 \\
35\end{array}$ & $\begin{array}{l}94 \\
18\end{array}$ & $\begin{array}{l}104 \\
20\end{array}$ & $\begin{array}{r}26 \\
5\end{array}$ & $\begin{array}{l}25 \\
10\end{array}$ & 27 & $\begin{array}{r}24 \\
8\end{array}$ & $\begin{array}{l}104 \\
22\end{array}$ & $\begin{array}{l}112 \\
31\end{array}$ & ${ }_{31}^{102}$ \\
\hline Provincial & 41 & $\begin{array}{r}59 \\
527\end{array}$ & 54 & 61 & 17 & 15 & 18 & 20 & 66 & 70 & 70 \\
\hline $\begin{array}{l}\text { Nontax revenue } \\
\text { Federal }\end{array}$ & $\begin{array}{l}414 \\
336\end{array}$ & $\begin{array}{l}527 \\
432\end{array}$ & $\begin{array}{l}507 \\
433\end{array}$ & $\begin{array}{l}515 \\
472\end{array}$ & $\begin{array}{l}157 \\
142\end{array}$ & $\begin{array}{r}109 \\
85\end{array}$ & $\begin{array}{r}124 \\
95\end{array}$ & $\begin{array}{l}174 \\
146\end{array}$ & $\begin{array}{l}557 \\
457\end{array}$ & $\begin{array}{l}563 \\
468\end{array}$ & $\begin{array}{l}563 \\
468\end{array}$ \\
\hline Provincial & & 95 & 74 & 43 & 15 & 24 & 29 & 28 & 90 & 95 & 95 \\
\hline $\begin{array}{l}\text { Grants } 1 / \\
\text { Of which: Tokyo pledges }\end{array}$ & 31 & 22 & 22 & 33 & $\begin{array}{l}34 \\
12\end{array}$ & $\begin{array}{l}34 \\
17\end{array}$ & $\begin{array}{l}28 \\
17\end{array}$ & $\begin{array}{r}8 \\
2 \\
2\end{array}$ & 31 & $\begin{array}{l}65 \\
46\end{array}$ & 104 \\
\hline Expenditure & 2,281 & 2,546 & 2,508 & 2,493 & 684 & 696 & 687 & 800 & 2,746 & 2877 & $2867+3$ \\
\hline Current expenditure & 1,858 & 2,147 & 2,088 & 2,131 & 576 & 578 & 542 & 631 & 2,176 & 2,261 & 2,327 \\
\hline Federal & $\begin{array}{r}1,420 \\
4,00\end{array}$ & $\begin{array}{l}1,630 \\
1618\end{array}$ & 1,571 & 1,582 & 440 & 436 & 395 & 466 & 1,581 & 1,671 & 1,737 \\
\hline $\begin{array}{l}\text { Interest } \\
\text { Domestic }\end{array}$ & $\begin{array}{l}490 \\
\ldots\end{array}$ & $\begin{array}{l}638 \\
538\end{array}$ & $\begin{array}{l}6199 \\
544\end{array}$ & $\begin{array}{l}628 \\
555\end{array}$ & $\begin{array}{l}149 \\
134\end{array}$ & $\begin{array}{l}167 \\
145\end{array}$ & $\begin{array}{l}149 \\
135\end{array}$ & $\begin{array}{l}184 \\
163\end{array}$ & $\begin{array}{l}678 \\
594\end{array}$ & $\begin{array}{l}677 \\
577\end{array}$ & $\begin{array}{l}649 \\
577\end{array}$ \\
\hline Foreign 21 & & 80 & 75 & $\begin{array}{l}505 \\
73\end{array}$ & 15 & 22 & 14 & 21 & 84 & 70 & 72 \\
\hline $\begin{array}{l}\text { Other } 3 / \\
\text { Of which: Subsidies and grants }\end{array}$ & 931 & $\begin{array}{l}1,012 \\
492\end{array}$ & $\begin{array}{l}952 \\
429\end{array}$ & $\begin{array}{l}954 \\
422\end{array}$ & $\begin{array}{l}291 \\
159\end{array}$ & $\begin{array}{l}269 \\
132\end{array}$ & $\begin{array}{l}246 \\
108\end{array}$ & $\begin{array}{l}282 \\
111\end{array}$ & $\begin{array}{l}903 \\
326\end{array}$ & 1,024 & 1,088 \\
\hline $\begin{array}{l}\text { Of which: Subsidies and grants } \\
\text { of which: Cash transfers to poor households } 4 /\end{array}$ & & & $\begin{array}{r}429 \\
46\end{array}$ & $\begin{array}{r}422 \\
38\end{array}$ & $\begin{array}{c}159 \\
13\end{array}$ & $\begin{array}{l}132 \\
17\end{array}$ & $\begin{array}{c}108 \\
25\end{array}$ & $\begin{array}{r}111 \\
29\end{array}$ & $\begin{array}{l}326 \\
83\end{array}$ & $\begin{array}{r}446 \\
84\end{array}$ & $\begin{array}{r}510 \\
84\end{array}$ \\
\hline Provincial & 437 & 517 & 517 & 549 & 136 & 142 & 148 & 165 & $\begin{array}{r}83 \\
595\end{array}$ & $\begin{array}{r}84 \\
590\end{array}$ & $\begin{aligned} 84 \\
590\end{aligned}$ \\
\hline Development expenditure and net lending & 423 & 399 & 420 & 361 & 109 & 118 & 145 & 169 & 569 & 617 & 540 \\
\hline $\begin{array}{l}\text { Public Sector Development Program } \\
\text { Federal } 21\end{array}$ & $\begin{array}{l}432 \\
238\end{array}$ & $\begin{array}{l}397 \\
247\end{array}$ & 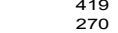 & $\begin{array}{l}356 \\
172\end{array}$ & $\begin{array}{r}106 \\
66\end{array}$ & $\begin{array}{r}115 \\
69\end{array}$ & $\begin{array}{r}142 \\
92\end{array}$ & $\begin{array}{l}166 \\
102\end{array}$ & $\begin{array}{l}558 \\
358\end{array}$ & $\begin{array}{l}606 \\
406\end{array}$ & $\begin{array}{l}530 \\
530\end{array}$ \\
\hline $\begin{array}{l}\text { Of which: One-off expenditure } \\
\text { Provincial }\end{array}$ & $\begin{array}{l}17 \\
214\end{array}$ & $\begin{array}{l}27 \\
150\end{array}$ & $\begin{array}{r}27 \\
149\end{array}$ & $\begin{array}{r}27 \\
184\end{array}$ & $\begin{array}{r}6 \\
40\end{array}$ & $\begin{array}{r}6 \\
46\end{array}$ & $\begin{array}{r}6 \\
50\end{array}$ & $\begin{array}{r}6 \\
64\end{array}$ & $\begin{array}{r}15 \\
210\end{array}$ & $\begin{array}{r}0 \\
200\end{array}$ & $\begin{array}{r}25 \\
200\end{array}$ \\
\hline $\begin{array}{l}\text { Other development expenditure } \\
\text { Net lending }\end{array}$ & -28 & 2 & 2 & 6 & 3 & 3 & 3 & 3 & 2 & 11 & 11 \\
\hline Statistical discrepancy ("+" = additional expenditure) 5/ & -3 & 11 & 27 & 45 & o & o & o & o & o & o & 0 \\
\hline Overall Deficit (excluding grants & -779 & -562 & -562 & -683 & -194 & -190 & -189 & -148 & -491 & -721 & -721 \\
\hline $\begin{array}{l}\text { Overall Deficit (including IIP gra } \\
\text { Overall Deficit (including grants) }\end{array}$ & -748 & -540 & -540 & -650 & $\begin{array}{r}-175 \\
-159\end{array}$ & $\begin{array}{l}-177 \\
-156\end{array}$ & $\begin{array}{l}-186 \\
-162\end{array}$ & $\begin{array}{l}-144 \\
-140\end{array}$ & -460 & -656 & -682 \\
\hline Financing $2 /$ & 748 & 540 & 540 & 650 & 159 & 156 & 162 & 140 & 460 & 656 & 617 \\
\hline $\begin{array}{l}\text { External } \\
\text { Of which: Tokyo pledges }\end{array}$ & 121 & 328 & 177 & 117 & $\begin{array}{r}100 \\
17\end{array}$ & $\begin{array}{l}47 \\
53\end{array}$ & $\begin{array}{l}55 \\
57\end{array}$ & $\begin{array}{l}66 \\
34\end{array}$ & 140 & 312 & $\begin{array}{l}267 \\
160\end{array}$ \\
\hline $\begin{array}{l}\text { Of which: IMF } \\
\text { Domestic }\end{array}$ & & 211 & & & $\begin{array}{l}59 \\
60\end{array}$ & $\begin{array}{r}30 \\
109\end{array}$ & $\begin{array}{r}30 \\
107\end{array}$ & $\begin{array}{r}-119 \\
74\end{array}$ & 320 & 343 & $\begin{array}{r}0 \\
350\end{array}$ \\
\hline $\begin{array}{l}\text { Domestic } \\
\text { Bank }\end{array}$ & 520 & 91 & 269 & 306 & 32 & 65 & 60 & 37 & 238 & 97 & 194 \\
\hline $\begin{array}{l}\text { Nank } \\
\text { Nonbank }\end{array}$ & 106 & 120 & 94 & 227 & 28 & 44 & 47 & 38 & 82 & 246 & 156 \\
\hline Privatization receipts & & & & & & & & & & & \\
\hline $\begin{array}{l}\text { Memorandum } \\
\text { Expenditure }\end{array}$ & 2,295 & & & 2,564 & 690 & 702 & 694 & 806 & 2,761 & 2.877 & 2.892 \\
\hline $\begin{array}{l}\text { Exer } \\
\text { Prima }\end{array}$ & $\begin{array}{l}2,289 \\
-289\end{array}-\mathrm{l} \cdot \mathrm{T}$ & $\begin{aligned} 2,584 \\
56\end{aligned}$ & $\begin{array}{lll}2,502 \\
57\end{array}$ & 年, -55 & -45 & -23 & $\begin{array}{l}-44 \\
-40\end{array}$ & 36 & 187 & -74 & 2,892 -72 \\
\hline Primary balan & -259 & 78 & 79 & -22 & -10 & 11 & -12 & 44 & 219 & -8 & 32 \\
\hline uding grants) $7 /$ & 285 & 308 & 318 & 333 & $\begin{array}{r}410 \\
80\end{array}$ & $\begin{array}{r}-251 \\
81\end{array}$ & $\begin{array}{r}-189 \\
79\end{array}$ & $\begin{array}{r}-148 \\
102\end{array}$ & & 343 & $\begin{array}{l}-998 \\
343\end{array}$ \\
\hline $\begin{array}{l}\text { Defense spending } \\
\text { Total government debt } 8 /\end{array}$ & 6,010 & 7,312 & 7,383 & 7,328 & 80 & & 19 & 102 & 7,916 & 343 & 30 \\
\hline Domestic det & 3,266 & 3,710 & 3,769 & 3,929 & & ... & & $\ldots$ & 4,164 & $\cdots$ & 4,637 \\
\hline $\begin{array}{l}\text { External debt 8/ } \\
\text { Nominal GDP (market prices) }\end{array}$ & $\begin{array}{r}2,744 \\
102884\end{array}$ & $\begin{array}{r}3,602 \\
13,384\end{array}$ & $\begin{array}{r}3,614 \\
12,970\end{array}$ & $\begin{array}{r}3,399 \\
13095\end{array}$ & 14.837 & 14.837 & 14.837 & 14.837 & $\begin{array}{r}3,752 \\
14,298\end{array}$ & 14.837 & $\begin{array}{r}3,793 \\
14837\end{array}$ \\
\hline
\end{tabular}

Sources: Pakistani authoritios for historical data; and Fund staff for estimates and projections

1/ For 2009/10 projections, this figures includes unidentified IDP-grants in the amount of $\$ 404$ million.

$3 / \ln 2007 / 08$, PR 23 billion (0.2 percent of GDP) of current expenditure has been reclassified as development expenditure.

$4 /$ Comprises BISP, Bait-ul-Mal, and Pakistan Poverty Alleviation Fund.
$5 /$ The statistical discrepancy is believed to arise mainly from double-counting of spending at the provincial level.

6/ Includes statistical discrepancy and spending related to the 2005 earthquake.

. 
Table 3b. Pakistan: Consolidated Government Budget, 2007/08-2009/10

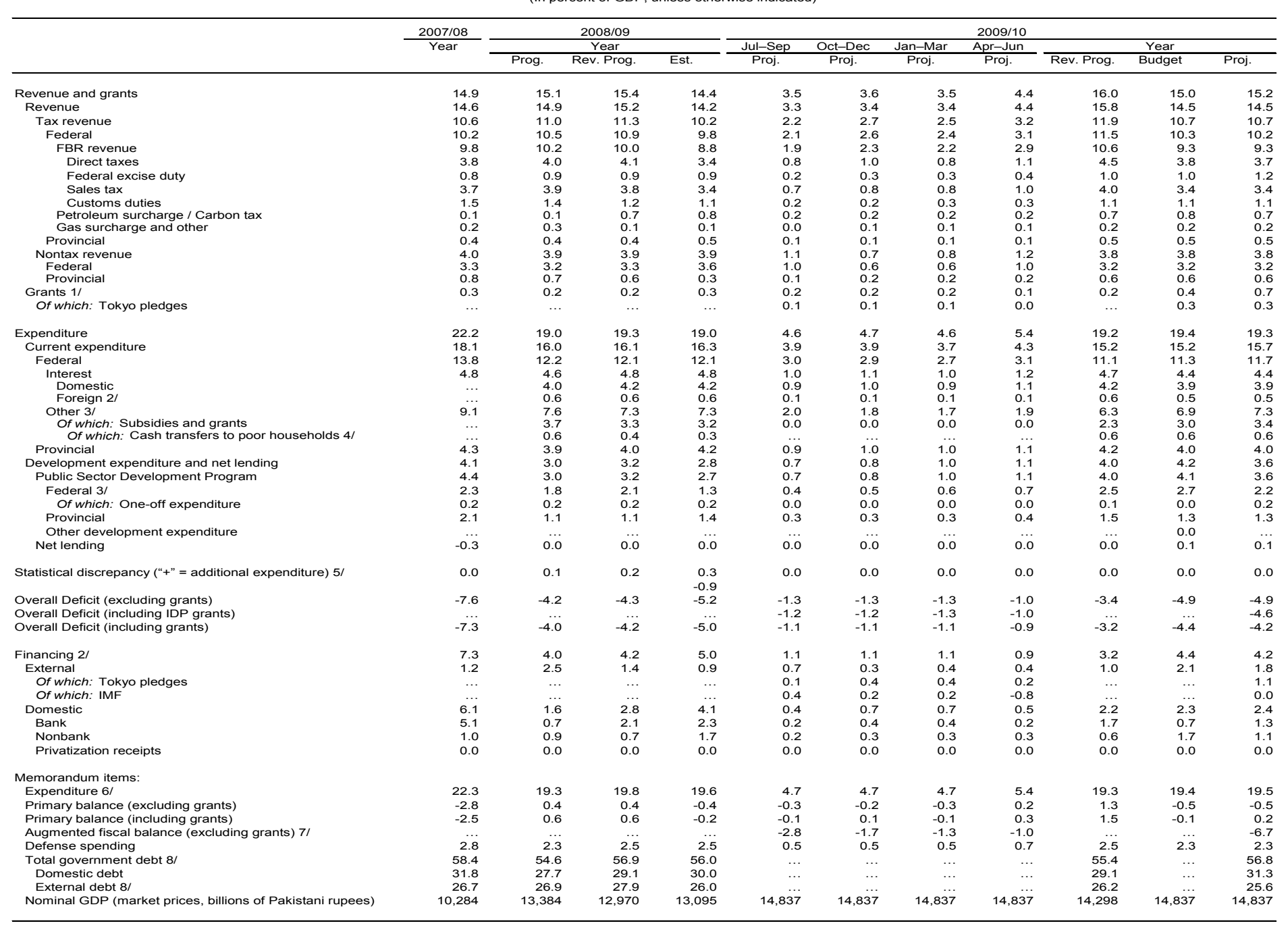

Sources: Pakistani authorities for historical data; and Fund staff for estimates and projections.

1/ For 2009/10 projections, this figures includes unidentified IDP-grants in the amount of $\$ 404$ million.

$2 /$ For $2007 / 08$, financing figures are provided by the Ministry of Finance.
$3 /$ In $2007 / 08, P R 23$ billion (0.2 percent of GDP of current expenditure has been reclassified as development expenditure.
$4 /$ Coprises

5/ The statistical discrepancy is believed to arise mainly from double-counting of spending at the provincial level.

$6 /$ Includes statistical discrepancy and spending related to the 2005 earthquake.

$7 /$ Reflects assumption of electricity sector debt by the budget.
$8 /$ Excludes military debt, commercial loans, and short-term debt. 
Table 4. Pakistan: Monetary Survey and Analytical Balance Sheet of the State Bank of Pakistan, 2007/08-2009/10

\begin{tabular}{|c|c|c|c|c|c|c|c|c|}
\hline & \multirow[t]{3}{*}{$2007 / 08$} & \multirow{2}{*}{\multicolumn{2}{|c|}{$\frac{2008 / 09}{\text { Jun. }}$}} & \multicolumn{5}{|c|}{$2009 / 10$} \\
\hline & & & & \multirow[t]{2}{*}{ Sep. } & \multirow{2}{*}{$\begin{array}{l}\text { Dec. } \\
\text { Proj. }\end{array}$} & \multirow[t]{2}{*}{ Mar. } & \multicolumn{2}{|c|}{ Jun. } \\
\hline & & Rev. Prog. & Est. & & & & Rev. Prog. & Proj. \\
\hline & \multicolumn{8}{|c|}{ (In billions of Pakistani rupees) } \\
\hline \multicolumn{9}{|l|}{ Monetary survey } \\
\hline Net foreign assets (NFA) & 668 & 509 & 517 & 560 & 597 & 601 & 436 & 617 \\
\hline Net domestic assets (NDA) & 4,022 & 4,574 & 4,621 & 4,695 & 4,826 & 4,901 & 5,187 & 5,189 \\
\hline Net claims on government, of which: & 1,473 & 1,803 & 1,998 & 2,008 & 2,047 & 2,081 & 2,051 & 2,188 \\
\hline Budget support & 1,325 & 1,595 & 1,631 & 1,663 & 1,728 & 1,788 & 1,833 & 1,825 \\
\hline Commodity operations & 127 & 227 & 336 & 315 & 289 & 263 & 237 & 382 \\
\hline Credit to nongovernment & 3,018 & 3,340 & 3,190 & 3,280 & 3,484 & 3,532 & 3,805 & 3,722 \\
\hline Private sector & 2,904 & 3,145 & 2,924 & 2,989 & 3,169 & 3,192 & 3,590 & 3,357 \\
\hline Public sector enterprises & 114 & 195 & 266 & 291 & 316 & 340 & 215 & 365 \\
\hline Privatization account & -3 & -3 & -3 & -3 & -3 & -3 & -3 & -3 \\
\hline Other items, net & -466 & -566 & -564 & -590 & -702 & -710 & -666 & -717 \\
\hline Broad money & 4,689 & 5,082 & 5,138 & 5,255 & 5,423 & 5,501 & 5,623 & 5,806 \\
\hline Currency & 982 & 1,192 & 1,152 & 1,147 & 1,288 & 1,274 & 1,245 & 1,275 \\
\hline Rupee deposits & 3,443 & 3,587 & 3,706 & 3,821 & 3,843 & 3,929 & 4,035 & 4,227 \\
\hline Foreign currency deposits & 263 & 303 & 280 & 286 & 292 & 298 & 343 & 303 \\
\hline \multicolumn{9}{|l|}{ State Bank of Pakistan (SBP) } \\
\hline NFA & 480 & 280 & 324 & 303 & 332 & 324 & 210 & 345 \\
\hline NDA & 1,000 & 1,241 & 1,183 & 1,256 & 1,282 & 1,306 & 1,466 & 1,346 \\
\hline Net claims on government & 1,015 & 1,167 & 1,144 & 1,144 & 1,144 & 1,144 & 1,167 & 1,144 \\
\hline Of which: budget support & 1,016 & 1,181 & 1,130 & 1,130 & 1,130 & 1,130 & 1,181 & 1,130 \\
\hline Claims on nongovernment & -7 & -7 & -7 & -7 & -7 & -7 & -7 & -7 \\
\hline Claims on scheduled banks & 227 & 327 & 303 & 316 & 329 & 342 & 357 & 355 \\
\hline Privatization account & -3 & -3 & -3 & -3 & -3 & -3 & -3 & -3 \\
\hline Other items, net & -231 & -242 & -254 & -194 & -182 & -170 & -48 & -115 \\
\hline Reserve money, of which: & 1,480 & 1,522 & 1,508 & 1,559 & 1,614 & 1,630 & 1,675 & 1,690 \\
\hline Banks' reserves & 425 & 256 & 274 & 281 & 323 & 326 & 357 & 333 \\
\hline Currency & 1,051 & 1,261 & 1,229 & 1,279 & 1,291 & 1,304 & 1,314 & 1,352 \\
\hline & \multicolumn{8}{|c|}{ (Annual percentage change, unless otherwise indicated) } \\
\hline Broad money & 15.3 & 8.4 & 9.6 & 12.4 & 13.2 & 14.5 & 10.6 & 13.0 \\
\hline NFA, banking system (in percent of broad money) 1/ & -7.8 & -3.4 & -3.2 & 1.6 & 4.6 & 2.9 & -1.4 & 1.9 \\
\hline NDA, banking system (in percent of broad money) $1 /$ & 23.2 & 11.8 & 12.8 & 10.8 & 8.6 & 11.6 & 12.1 & 11.1 \\
\hline Budgetary support (in percent of broad money) 1/ & 12.5 & 5.7 & 6.5 & 5.0 & 4.6 & 6.0 & 4.7 & 3.8 \\
\hline NFA, banking system & -32.2 & -23.8 & -22.5 & 15.3 & 58.9 & 29.8 & -14.3 & 19.2 \\
\hline NDA, banking system & 30.6 & 13.7 & 14.9 & 12.1 & 9.3 & 12.8 & 13.4 & 12.3 \\
\hline Budgetary support & 62.4 & 20.3 & 23.1 & 16.3 & 14.7 & 19.1 & 14.9 & 11.9 \\
\hline Private credit & 16.4 & 8.3 & 0.7 & -0.2 & 2.0 & 7.1 & 14.1 & 14.8 \\
\hline Currency & 16.9 & 21.3 & 17.3 & 3.6 & 14.6 & 14.3 & 4.4 & 10.7 \\
\hline Reserve money & 22.3 & 2.8 & 1.9 & 0.0 & 10.8 & 12.1 & 10.1 & 12.1 \\
\hline NFA, SBP (in percent of reserve money) $1 /$ & -25.4 & -13.5 & -10.5 & -0.3 & 10.0 & 5.0 & -4.6 & 1.3 \\
\hline NDA, SBP (in percent of reserve money) $1 /$ & 47.7 & 16.3 & 12.4 & 0.4 & 0.8 & 7.2 & 14.7 & 10.8 \\
\hline Net claims on government (in percent of reserve money) $1 /$ & 56.9 & 10.3 & 8.7 & -6.1 & -6.3 & 3.4 & 0.0 & 0.0 \\
\hline \multicolumn{9}{|l|}{ Memorandum items: } \\
\hline Velocity & 2.2 & 2.6 & 2.5 & $\ldots$ & $\ldots$ & $\ldots$ & 2.5 & 2.6 \\
\hline Money multiplier & 3.2 & 3.3 & 3.4 & 3.4 & 3.4 & 3.4 & 3.4 & 3.4 \\
\hline Currency to broad money ratio (percent) & 20.9 & 23.4 & 22.4 & 21.8 & 23.7 & 23.2 & 22.1 & 22.0 \\
\hline Currency to deposit ratio (percent) & 26.5 & 30.6 & 28.9 & 27.9 & 31.1 & 30.2 & 28.4 & 28.1 \\
\hline Reserves to deposit ratio (percent) & 11.5 & 6.6 & 6.9 & 6.8 & 7.8 & 7.7 & 8.2 & 7.4 \\
\hline Budget bank financing (billions of Pakistani rupees), of which: & 509 & 269 & 306 & 32 & 97 & 157 & 238 & 194 \\
\hline By commercial banks & -167 & 104 & 192 & 32 & 97 & 157 & 238 & 194 \\
\hline By SBP & 677 & 165 & 114 & 0 & 0 & 0 & 0 & 0 \\
\hline NFA of SBP (change from beginning of the year in billions of U.S. dollars) $2 /$ & -4.5 & -2.4 & -3.1 & -0.3 & 0.0 & -0.1 & -0.8 & 0.1 \\
\hline NFA of commercial banks (billions of Pakistani rupees) & 187 & 228 & 193 & 257 & 265 & 276 & 226 & 272 \\
\hline NDA of commercial banks (billions of Pakistani rupees) & 3,022 & 3,332 & 3,438 & 3,439 & 3,544 & 3,595 & 3,721 & 3,844 \\
\hline Excess reserves in percent of broad money & 1.5 & $\ldots$ & $\ldots$ & $\ldots$ & $\ldots$ & $\ldots$ & $\ldots$ & $\ldots$ \\
\hline
\end{tabular}

Sources: Pakistani authorities for historical data; and Fund staff estimates and projections.

$1 /$ Denominator is the stock of broad (reserve) money at the end of the previous year.

2/ Includes valuation adjustments. 
Table 5. Pakistan: Medium-Term Macroeconomic Framework, 2007/08-2013/14

\begin{tabular}{|c|c|c|c|c|c|c|c|}
\hline & $2007 / 08$ & $\frac{2008 / 09}{\text { Est. }}$ & $2009 / 10$ & $2010 / 11$ & $\begin{array}{c}2011 / 12 \\
\text { Projection }\end{array}$ & $2012 / 13$ & $2013 / 14$ \\
\hline & \multicolumn{7}{|c|}{ (In percent of GDP) } \\
\hline \multicolumn{8}{|l|}{ Saving and investment } \\
\hline Current account balance & -8.3 & -5.1 & -4.7 & -5.3 & -5.0 & -4.3 & -3.4 \\
\hline Gross national saving & 13.6 & 14.6 & 14.3 & 15.8 & 16.5 & 17.2 & 18.8 \\
\hline Government saving & -2.9 & -2.2 & -0.6 & 0.6 & 1.7 & 2.1 & 2.6 \\
\hline Non-government saving & 16.5 & 16.8 & 14.9 & 15.1 & 14.9 & 15.1 & 16.2 \\
\hline Gross capital formation & 22.0 & 19.7 & 19.0 & 21.1 & 21.6 & 21.6 & 22.2 \\
\hline Government & 4.4 & 2.7 & 3.6 & 4.5 & 4.7 & 4.9 & 5.1 \\
\hline Nongovernment (including public sector enterprises) & 17.6 & 17.0 & 15.5 & 16.6 & 16.8 & 16.7 & 17.1 \\
\hline & \multicolumn{7}{|c|}{ (In billions of U.S. dollars, unless otherwise indicated) } \\
\hline \multicolumn{8}{|l|}{ Balance of payments } \\
\hline Current account balance & -13.7 & -8.6 & -8.5 & -9.7 & -9.8 & -8.9 & -7.5 \\
\hline Net capital flows $1 /$ & 1.3 & 1.3 & 1.3 & 1.5 & 1.7 & 1.7 & 1.8 \\
\hline Of which: foreign direct investment $2 /$ & 5.4 & 3.5 & 3.0 & 3.5 & 4.0 & 4.5 & 5.0 \\
\hline Gross official reserves & 8.6 & 9.1 & 13.5 & 15.1 & 14.6 & 13.1 & 12.1 \\
\hline In months of imports $3 /$ & 2.7 & 2.9 & 4.1 & 4.4 & 4.1 & 3.5 & 3.1 \\
\hline \multirow[t]{2}{*}{ External debt (in percent of GDP) } & 27.0 & 29.9 & 32.1 & 35.1 & 35.5 & 34.5 & 32.5 \\
\hline & \multicolumn{7}{|c|}{ (In percent of GDP) } \\
\hline \multicolumn{8}{|l|}{ Public finances } \\
\hline Revenue and grants & 14.9 & 14.4 & 15.2 & 15.4 & 16.0 & 16.3 & 16.6 \\
\hline Of which: tax revenue & 10.6 & 10.2 & 10.7 & 11.4 & 12.2 & 12.7 & 13.0 \\
\hline Expenditure (incl. stat. discr.), of which: & 22.2 & 19.4 & 19.3 & 19.3 & 19.0 & 19.0 & 19.2 \\
\hline Current & 18.1 & 16.3 & 15.7 & 14.8 & 14.3 & 14.2 & 14.0 \\
\hline Development (incl. net-lending) & 4.1 & 2.8 & 3.6 & 4.5 & 4.7 & 4.9 & 5.2 \\
\hline Primary balance 4/ & -2.5 & -0.2 & 0.2 & 0.0 & 0.4 & 0.3 & 0.1 \\
\hline Overall fiscal balance 4/ & -7.3 & -5.0 & -4.2 & -3.8 & -3.0 & -2.8 & -2.6 \\
\hline \multirow[t]{2}{*}{ Total government debt } & 58.4 & 56.0 & 56.8 & 57.1 & 57.1 & 56.8 & 56.3 \\
\hline & \multicolumn{7}{|c|}{ (Annual changes in percent) } \\
\hline \multicolumn{8}{|l|}{ Output and prices } \\
\hline Real GDP at factor cost & 4.1 & 2.0 & 3.0 & 4.0 & 4.5 & 5.0 & 5.5 \\
\hline Consumer prices (period average) & 12.0 & 20.8 & 10.0 & 7.0 & 6.0 & 6.0 & 6.0 \\
\hline \multicolumn{8}{|l|}{ Memorandum items } \\
\hline Real per capita consumption (percentage change) & 5.6 & 7.5 & 1.3 & 2.5 & 2.5 & 2.5 & 2.5 \\
\hline
\end{tabular}

Sources: Pakistani authorities for historical data; and Fund staff estimates and projections.

1/ Difference between the overall balance and the current account balance.

2/ Including privatization.

3 / In months of next year's imports of goods and services.

$4 /$ Including grants and earthquake-related expenditures. 
Table 6. Pakistan: Medium-Term Balance of Payments, 2007/08-2013/14

(In millions of U.S. dollars; unless otherwise indicated)

\begin{tabular}{|c|c|c|c|c|c|c|c|}
\hline & $2007 / 08$ & $2008 / 09$ & $2009 / 10$ & $2010 / 11$ & $2011 / 12$ & $2012 / 13$ & $2013 / 14$ \\
\hline & & Est. & \multicolumn{5}{|c|}{ Projection } \\
\hline Current account & $-13,735$ & $-8,551$ & $-8,487$ & $-9,748$ & $-9,765$ & $-8,946$ & $-7,538$ \\
\hline Balance on goods & $-15,045$ & $-11,965$ & $-11,692$ & $-12,509$ & $-12,740$ & $-12,564$ & $-12,345$ \\
\hline Exports, f.o.b. & 20,427 & 18,915 & 18,441 & 19,072 & 20,089 & 21,518 & 23,170 \\
\hline Imports, f.o.b. & $-35,472$ & $-30,880$ & $-30,133$ & $-31,580$ & $-32,829$ & $-34,082$ & $-35,515$ \\
\hline Services (net) & $-6,257$ & $-3,180$ & $-2,454$ & $-3,339$ & $-3,644$ & $-3,783$ & $-3,937$ \\
\hline Services: credit & 3,577 & 4,017 & 4,550 & 4,209 & 4,428 & 4,746 & 5,110 \\
\hline Services: debit & $-9,834$ & $-7,197$ & $-7,005$ & $-7,548$ & $-8,072$ & $-8,529$ & $-9,048$ \\
\hline Income (net) & $-3,909$ & $-4,471$ & $-4,333$ & $-4,424$ & $-4,726$ & $-4,940$ & $-4,819$ \\
\hline Income: credit & 1,613 & 898 & 909 & 921 & 953 & 999 & 1,048 \\
\hline Income: debit & $-5,522$ & $-5,369$ & $-5,241$ & $-5,345$ & $-5,678$ & $-5,939$ & $-5,866$ \\
\hline Of which: interest payments & $-2,161$ & $-1,913$ & $-1,850$ & $-1,732$ & $-1,849$ & $-1,884$ & $-1,811$ \\
\hline Of which: income on direct investment & $-3,361$ & $-3,456$ & $-3,391$ & $-3,613$ & $-3,830$ & $-4,055$ & $-4,301$ \\
\hline Balance on goods, services, and income & $-25,211$ & $-19,616$ & $-18,479$ & $-20,272$ & $-21,109$ & $-21,287$ & $-21,102$ \\
\hline Current transfers (net) & 11,476 & 11,065 & 9,992 & 10,524 & 11,345 & 12,342 & 13,564 \\
\hline Current transfers: credit, of which:1/ & 11,618 & 11,174 & 10,050 & 10,632 & 11,453 & 12,450 & 13,672 \\
\hline Official & 484 & 158 & 464 & 375 & 375 & 375 & 390 \\
\hline Workers' remittances & 6,451 & 7,751 & 6,700 & 7,169 & 7,743 & 8,439 & 9,283 \\
\hline Other private transfers & 4,683 & 3,265 & 2,886 & 3,088 & 3,335 & 3,635 & 3,999 \\
\hline Current transfers: debit & -142 & -109 & -58 & -108 & -108 & -108 & -108 \\
\hline Capital account & 121 & 164 & 964 & 385 & 208 & 214 & 220 \\
\hline Capital transfers: credit & 128 & 166 & 967 & 388 & 211 & 217 & 223 \\
\hline Of which: official capital grants & 111 & 141 & 952 & 279 & 100 & 100 & 100 \\
\hline Of which: Tokyo pledges & $\ldots$ & $\ldots$ & 562 & 822 & 548 & $\ldots$ & $\ldots$ \\
\hline Capital transfers: debit & -7 & -2 & -3 & -3 & -3 & -3 & -3 \\
\hline Financial account & 8,182 & 4,996 & 7,625 & 9,306 & 10,582 & 10,559 & 11,057 \\
\hline Direct investment abroad & -75 & -23 & -30 & -50 & -10 & -9 & -8 \\
\hline Direct investment in Pakistan & 5,410 & 3,476 & 2,980 & 3,500 & 4,000 & 4,500 & 5,000 \\
\hline Of which: privatization receipts & 133 & 0 & 266 & 300 & 300 & 300 & 200 \\
\hline Portfolio investment (net), of which: & 36 & $-1,218$ & -484 & 478 & 1,300 & 1,500 & 1,500 \\
\hline Eurobond/GDR & -25 & -525 & -622 & -22 & 500 & 500 & 500 \\
\hline Other investment assets & 32 & 836 & -284 & -448 & -460 & -473 & -486 \\
\hline General government & 5 & 7 & -8 & -8 & -8 & -8 & -8 \\
\hline Banks & 525 & 327 & -200 & -200 & -200 & -200 & -200 \\
\hline Other sectors & -498 & 502 & -76 & -240 & -252 & -265 & -278 \\
\hline Other investment liabilities & 2,779 & 1,925 & 5,443 & 5,825 & 5,752 & 5,041 & 5,051 \\
\hline Monetary authorities & 490 & 0 & 1,267 & 0 & 0 & 0 & 0 \\
\hline General government, of which: & 1,808 & 1,976 & 3,526 & 4,935 & 4,844 & 4,320 & 4,244 \\
\hline Disbursements $1 /$ & 3,078 & 4,313 & 5,408 & 6,575 & 6,275 & 5,875 & 5,860 \\
\hline Of which: Tokyo pledges & $\ldots$ & $\ldots$ & 1,928 & 1,113 & 742 & & \\
\hline Amortization & $-1,249$ & $-2,337$ & $-1,882$ & $-1,640$ & $-1,431$ & $-1,555$ & $-1,616$ \\
\hline Banks & 66 & -587 & 100 & 100 & 100 & 100 & 100 \\
\hline Other sectors & 415 & 536 & 550 & 791 & 808 & 621 & 706 \\
\hline Net errors and omissions & -356 & 99 & 0 & 0 & 0 & 0 & 0 \\
\hline Reserves and related items & 5,788 & 3,292 & -102 & 57 & $-1,025$ & $-1,828$ & $-3,739$ \\
\hline Reserve assets, of which: & 5,961 & -457 & $-4,460$ & $-1,980$ & 100 & 1,080 & 550 \\
\hline Foreign exchange (State Bank of Pakistan) & 5,711 & -523 & $-4,360$ & $-1,600$ & 500 & 1,500 & 1,000 \\
\hline Foreign exchange (deposit money banks) & 250 & 66 & -100 & -380 & -400 & -420 & -450 \\
\hline Use of Fund credit and loans & -173 & 3,749 & 4,358 & 2,037 & $-1,125$ & $-2,908$ & $-4,289$ \\
\hline Exceptional financing & 0 & 0 & 0 & 0 & 0 & 0 & 0 \\
\hline \multicolumn{8}{|l|}{ Memorandum items: } \\
\hline Current account (in percent of GDP; including official transfers) & -8.3 & -5.1 & -4.7 & -5.3 & -5.0 & -4.3 & -3.4 \\
\hline Current account (in percent of GDP; excluding fuel imports) & -2.0 & 0.8 & 1.0 & 1.0 & 1.3 & 2.0 & 2.9 \\
\hline Exports f.o.b. (growth rate, in percent) & 18.2 & -7.4 & -2.5 & 3.4 & 5.3 & 7.1 & 7.7 \\
\hline Imports f.o.b. (growth rate, in percent) & 31.4 & -12.9 & -2.4 & 4.8 & 4.0 & 3.8 & 4.2 \\
\hline Imports f.o.b. (growth rate, in percent, excluding oil) & 26.8 & -15.3 & -5.5 & 1.5 & 2.4 & 2.5 & 3.0 \\
\hline Oil imports (in million U.S.\$, cif) & 10,495 & 9,850 & 10,333 & 11,556 & 12,364 & 13,132 & 13,960 \\
\hline Crude oil price $(\$ / b b l)$ & 87.0 & 68.5 & 70.0 & 79.1 & 81.9 & 83.6 & 84.9 \\
\hline \multicolumn{8}{|l|}{ Workers' remittances and other private transfers } \\
\hline (growth rate, in percent) & 10.1 & -1.1 & -13.0 & 7.0 & 8.0 & 9.0 & 10.0 \\
\hline External debt (in millions of U.S. dollars) & 44,468 & 49,860 & 57,417 & 64,607 & 68,994 & 70,955 & 71,338 \\
\hline Gross financing needs (in millions of U.S. dollars) $2 /$ & 15,066 & 11,536 & 11,246 & 11,691 & 12,344 & 13,431 & 13,465 \\
\hline \multirow{2}{*}{$\begin{array}{l}\text { End-period gross official reserves (millions of U.S. dollars) 3/ } \\
\text { (In months of next year's imports of goods and services) }\end{array}$} & 8,591 & 9,114 & 13,474 & 15,074 & 14,574 & 13,074 & 12,074 \\
\hline & 2.7 & 2.9 & 4.1 & 4.4 & 4.1 & 3.5 & 3.1 \\
\hline GDP (in millions of U.S. dollars) & 164,557 & 166,513 & 178,723 & 184,151 & 194,270 & 205,926 & 219,322 \\
\hline
\end{tabular}

Sources: Pakistani authorities; and Fund staff estimates and projections.

1/ For 2009/10, includes $\$ 1$ billion from the U.S. Kerry-Lugar support. For 20010/11-2013/14, includes total U.S. Kerry-Lugar support of $\$ 6$ billon of which 15 percent assumed to be current transfers, and 85 percent as project support.

2/ Defined as current account deficit, plus amortization on medium- and long-term debt, plus short-term debt at end of previous period.

3/ Excluding foreign currency deposits held with the State Bank of Pakistan (cash reserve requirements) and gold. 
Table 7. Pakistan: Medium-Term Fiscal Framework, 2007/08-2013/14

(In percent of GDP; unless otherwise indicated)

\begin{tabular}{|c|c|c|c|c|c|c|c|}
\hline & $2007 / 08$ & $\frac{2008 / 09}{\text { Est. }}$ & $2009 / 10$ & $2010 / 11$ & $\frac{2011 / 12}{\text { Projection }}$ & $2012 / 13$ & $2013 / 14$ \\
\hline Revenue and grants & 14.9 & 14.4 & 15.2 & 15.4 & 16.0 & 16.3 & 16.6 \\
\hline Tax revenue & 10.6 & 10.2 & 10.7 & 11.4 & 12.2 & 12.7 & 13.0 \\
\hline Of which: Federal Board of Revenue & 9.8 & 8.8 & 9.3 & 10.1 & 10.9 & 11.5 & 11.9 \\
\hline Nontax revenue & 4.0 & 3.9 & 3.8 & 3.7 & 3.5 & 3.3 & 3.3 \\
\hline Grants & 0.3 & 0.3 & 0.7 & 0.4 & 0.2 & 0.2 & 0.2 \\
\hline Expenditure & 22.2 & 19.0 & 19.3 & 19.3 & 19.0 & 19.0 & 19.2 \\
\hline Current expenditure & 18.1 & 16.3 & 15.7 & 14.8 & 14.3 & 14.2 & 14.0 \\
\hline Interest & 4.8 & 4.8 & 4.4 & 3.8 & 3.4 & 3.1 & 2.7 \\
\hline Other federal & 9.1 & 7.3 & 7.3 & 6.5 & 6.3 & 6.5 & 6.7 \\
\hline Provincial & 4.3 & 4.2 & 4.0 & 4.4 & 4.5 & 4.6 & 4.6 \\
\hline Development expenditure & 4.4 & 2.7 & 3.6 & 4.5 & 4.7 & 4.9 & 5.1 \\
\hline Of which: one-off outlays $1 /$ & 0.2 & 0.2 & 0.2 & 0.0 & 0.0 & 0.0 & 0.0 \\
\hline Net-lending & -0.3 & 0.0 & 0.1 & 0.0 & 0.0 & 0.0 & 0.0 \\
\hline Statistical discrepancy & 0.0 & 0.3 & 0.0 & 0.0 & 0.0 & 0.0 & 0.0 \\
\hline \multicolumn{8}{|l|}{ Overall balance } \\
\hline Excluding grants & -7.6 & -5.2 & -4.9 & -4.2 & -3.3 & -3.0 & -2.8 \\
\hline Including grants & -7.3 & -5.0 & -4.2 & -3.8 & -3.0 & -2.8 & -2.6 \\
\hline Financing & 7.3 & 5.0 & 4.2 & 3.8 & 3.0 & 2.8 & 2.6 \\
\hline External & 1.2 & 0.9 & 1.8 & 2.8 & 2.9 & 2.5 & 2.3 \\
\hline Of which : privatization receipts & 0.0 & 0.0 & 0.1 & 0.2 & 0.2 & 0.1 & 0.1 \\
\hline Domestic & 6.1 & 4.1 & 2.4 & 1.0 & 0.1 & 0.3 & 0.3 \\
\hline \multicolumn{8}{|l|}{ Memorandum items: } \\
\hline \multicolumn{8}{|l|}{ Primary balance } \\
\hline Excluding grants & -2.8 & -0.4 & -0.5 & -0.4 & 0.1 & 0.1 & -0.1 \\
\hline Including grants & -2.5 & -0.2 & 0.2 & 0.0 & 0.4 & 0.3 & 0.1 \\
\hline Interest payments/revenue (ratio) & 32.7 & 33.9 & 30.2 & 25.3 & 21.8 & 19.0 & 16.6 \\
\hline Public debt $2 /$ & 58.4 & 56.0 & 56.8 & 57.1 & 57.1 & 56.8 & 56.3 \\
\hline External & 26.7 & 26.0 & 25.6 & 27.6 & 29.8 & 31.5 & 32.9 \\
\hline Domestic & 31.8 & 30.0 & 31.3 & 29.6 & 27.3 & 25.3 & 23.4 \\
\hline Public debt (including obligations to the IMF) & 59.3 & 59.0 & 62.2 & 63.5 & 62.7 & 60.6 & 57.8 \\
\hline Nominal GDP (billions of Pakistani rupees) & 10,284 & 13,095 & 14,837 & 16,510 & 18,288 & 20,355 & 22,763 \\
\hline
\end{tabular}

Sources: Pakistani authorities for historical data; and Fund staff for estimates and projections.

$1 /$ Spending related to the 2005 earthquake.

2/ Excludes obligations to the IMF, military debt, commercial loans, and short-term debt. 
Table 8. Pakistan: External Debt, 2007/08-2015/16

(In millions of U.S. dollars, outstanding as end of each fiscal year)

\begin{tabular}{|c|c|c|c|c|c|c|c|c|c|}
\hline & $2007 / 08$ & $2008 / 09$ & $2009 / 10$ & $2010 / 11$ & $2011 / 12$ & $2012 / 13$ & $2013 / 14$ & $2014 / 15$ & $2015 / 16$ \\
\hline & & Est. & \multicolumn{7}{|c|}{ Projection } \\
\hline Total external debt & 44,516 & 49,860 & 57,417 & 64,607 & 68,994 & 70,955 & 71,338 & 72,075 & 74,161 \\
\hline Public and publicly guaranteed debt (incl. IMF) & 41,629 & 46,685 & 53,947 & 60,897 & 65,116 & 67,029 & 67,484 & 68,300 & 70,437 \\
\hline Public and publicly guaranteed debt (excl. IMF) & 40,292 & 41,721 & 44,625 & 49,538 & 54,882 & 59,702 & 64,446 & 67,870 & 70,437 \\
\hline Medium- and long-term debt & 39,479 & 40,902 & 44,102 & 49,315 & 54,659 & 59,479 & 64,223 & 67,447 & 70,014 \\
\hline Multilateral creditors & 21,583 & 22,834 & 24,686 & 26,363 & 27,982 & 29,697 & 31,370 & 32,606 & 33,714 \\
\hline $\mathrm{ADB}$ & 9,401 & 10,517 & 11,627 & 12,883 & 14,082 & 15,141 & 16,159 & 17,143 & 18,109 \\
\hline World Bank & 11,532 & 11,704 & 12,440 & 12,894 & 13,345 & 14,030 & 14,716 & 14,997 & 15,166 \\
\hline Other & 651 & 613 & 618 & 585 & 555 & 526 & 495 & 466 & 439 \\
\hline Bilateral creditors & 15,181 & 15,914 & 17,888 & 21,446 & 24,671 & 27,276 & 29,847 & 31,335 & 32,794 \\
\hline Paris Club & 13,936 & 13,790 & 13,660 & 13,521 & 13,370 & 13,201 & 13,011 & 12,796 & 12,549 \\
\hline Non-paris club & 1,245 & 2,124 & 4,228 & 7,925 & 11,301 & 14,075 & 16,836 & 18,539 & 20,245 \\
\hline Bonds & 2,650 & 2,150 & 1,528 & 1,506 & 2,006 & 2,506 & 3,006 & 3,506 & 3,506 \\
\hline Of which: Euro bond & 2,650 & 2,150 & 1,528 & 1,506 & 2,006 & 2,506 & 3,006 & 3,506 & 3,506 \\
\hline Commercial banks and others & 65 & 4 & 0 & 0 & 0 & 0 & 0 & 0 & 0 \\
\hline Short-term debt & 813 & 819 & 523 & 223 & 223 & 223 & 223 & 423 & 423 \\
\hline Of which: IDB & 713 & 819 & 523 & 223 & 223 & 223 & 223 & 423 & 423 \\
\hline IMF & 1,337 & 4,964 & 9,322 & 11,359 & 10,234 & 7,327 & 3,038 & 430 & 0 \\
\hline Private sector debt & 2,887 & 3,175 & 3,470 & 3,709 & 3,878 & 3,926 & 3,854 & 3,774 & 3,724 \\
\hline \multicolumn{10}{|l|}{ Of which: } \\
\hline Multilateral creditors & 322 & $\ldots$ & $\ldots$ & $\ldots$ & $\ldots$ & $\ldots$ & $\ldots$ & $\ldots$ & $\ldots$ \\
\hline Paris Club creditors & 1,274 & $\ldots$ & $\ldots$ & $\ldots$ & $\ldots$ & $\ldots$ & $\ldots$ & $\ldots$ & $\ldots$ \\
\hline Non-paris club creditors & 9 & $\ldots$ & $\ldots$ & $\ldots$ & $\ldots$ & $\ldots$ & $\ldots$ & $\ldots$ & $\ldots$ \\
\hline Other & 1,007 & $\ldots$ & $\ldots$ & $\ldots$ & $\ldots$ & $\ldots$ & $\ldots$ & $\ldots$ & $\ldots$ \\
\hline
\end{tabular}

Sources: Pakistani authorities; and Fund staff estimates and projections. 
Table 9. Pakistan: Gross Financing Requirements and Sources, 2008/09-20010/11

(In millions of U.S. dollars; unless otherwise specified)

\begin{tabular}{|c|c|c|c|}
\hline \multirow{3}{*}{ Gross external financing requirements } & \multirow{3}{*}{$\begin{array}{r}2008 / 09 \\
11,573\end{array}$} & $2009 / 10$ & $2010 / 11$ \\
\hline & & \multicolumn{2}{|c|}{ Projection } \\
\hline & & 11,223 & 11,668 \\
\hline Current account deficit (if surplus $=-$ ) & 8,551 & 8,487 & 9,748 \\
\hline Of which: Net interest payments (if net receipts = -) & 1,913 & 1,850 & 1,732 \\
\hline Maturing short-term debt & 850 & 796 & 500 \\
\hline Amortization of medium- and long-term debt & 2,172 & 1,940 & 1,420 \\
\hline Medium and long-term to external private creditors & 525 & 622 & 22 \\
\hline Medium and long-term to external official creditors & 1,647 & 1,318 & 1,398 \\
\hline IMF & 160 & 232 & 258 \\
\hline To other official creditors & 1,487 & 1,086 & 1,140 \\
\hline Available financing & 11,573 & 9,434 & 10,363 \\
\hline Net FDI (including privatization receipts) & 3,453 & 2,950 & 3,450 \\
\hline Roll-over of short-term debt & 856 & 500 & 200 \\
\hline Medium- and long-term borrowing & 7,366 & 7,709 & 7,365 \\
\hline From external official creditors (currently identified) & 7,366 & 7,709 & 7,365 \\
\hline Borrowing from IMF (gross) & 3,909 & 2,801 & 990 \\
\hline Borrowing from other official creditors & 3,457 & 4,908 & 6,375 \\
\hline Other net capital inflows & 421 & 2,635 & 948 \\
\hline Of which: SDR allocation & $\ldots$ & 1,267 & 0 \\
\hline Reserve accumulation (decrease $=+$ ) & -523 & $-4,360$ & $-1,600$ \\
\hline Remaining financing gap & 0 & 1,790 & 1,305 \\
\hline \multicolumn{4}{|l|}{ Of which: } \\
\hline Proposed augmentation of Fund resources & $\ldots$ & 1,790 & 1,305 \\
\hline Other exceptional financing & $\cdots$ & $\cdots$ & $\cdots$ \\
\hline \multicolumn{4}{|l|}{ Memorandum items: } \\
\hline Gross international reserves in U.S.\$ billions & 9.1 & 13.5 & 15.1 \\
\hline In months of imports & 2.9 & 4.1 & 4.4 \\
\hline
\end{tabular}

Sources: State Bank of Pakistan, and Fund staff estimates and projections. 
Table 10. Pakistan: Indicators of Fund Credit, 2008/09-2015/16

(In millions of SDR unless otherwise specified)

\begin{tabular}{|c|c|c|c|c|c|c|c|c|}
\hline & $2008 / 09$ & $2009 / 10$ & $2010 / 11$ & $2011 / 12$ & $2012 / 13$ & $2013 / 14$ & $2014 / 15$ & $2015 / 16$ \\
\hline & Est. & \multicolumn{7}{|c|}{ Projection } \\
\hline & \multicolumn{8}{|c|}{ (Projected Debt Service to the Fund based on Existing and Prospective Drawings) } \\
\hline \multicolumn{9}{|l|}{ PRGF } \\
\hline Principal $1 /$ & 137.7 & 155.1 & 172.3 & 163.7 & 120.6 & 51.7 & 17.2 & 0.0 \\
\hline Interest and charges $1 /$ & 4.0 & 3.1 & 2.2 & 1.4 & 0.6 & 0.2 & 0.0 & 0.0 \\
\hline \multicolumn{9}{|l|}{ Stand-By Arrangements } \\
\hline Principal & 0.0 & 0.0 & 0.0 & 587.9 & 1821.9 & 2813.6 & 1725.0 & 287.5 \\
\hline \multirow[t]{2}{*}{ Interest and charges } & 42.9 & 85.1 & 167.0 & 183.2 & 165.3 & 77.4 & 18.9 & 2.2 \\
\hline & \multicolumn{8}{|c|}{ (Projected Level of Credit Outstanding based on Existing and Prospective Drawings') } \\
\hline Total & $3,316.4$ & $6,228.2$ & $7,589.1$ & $6,837.5$ & $4,895.0$ & $2,029.7$ & 287.5 & 0.0 \\
\hline PRGF Arrangements & 680.5 & 525.4 & 353.2 & 189.5 & 69.0 & 17.3 & 0.0 & 0.0 \\
\hline \multirow[t]{2}{*}{ Stand-by Arrangements } & $2,635.9$ & $5,702.7$ & $7,235.9$ & $6,648.0$ & $4,826.1$ & $2,012.4$ & 287.5 & 0.0 \\
\hline & & \multicolumn{7}{|c|}{ (Projected Debt Service to the Fund based on Existing and Prospective Drawings) } \\
\hline Total $1 /$ & 184.6 & 243.3 & 341.5 & 936.1 & $2,108.5$ & $2,942.9$ & $1,761.0$ & 289.6 \\
\hline \multicolumn{9}{|l|}{ Of which: } \\
\hline Principal & 137.7 & 155.1 & 172.3 & 751.6 & $1,942.5$ & $2,865.3$ & $1,742.2$ & 287.5 \\
\hline Interest and charges & 46.9 & 88.2 & 169.2 & 184.5 & 165.9 & 77.5 & 18.9 & 2.2 \\
\hline In percent of exports of goods and non-factor services & 1.2 & 1.6 & 2.2 & 5.7 & 12.0 & 15.6 & 8.7 & 1.3 \\
\hline In percent of GDP & 0.2 & 0.2 & 0.3 & 0.7 & 1.5 & 2.0 & 1.1 & 0.2 \\
\hline In percent of end-period gross international reserves & 3.0 & 2.7 & 3.4 & 9.6 & 24.1 & 36.5 & 19.1 & 2.7 \\
\hline \multicolumn{9}{|l|}{ Memorandum items } \\
\hline Exports of goods and NFS (in millions of U.S. dollars) & 22,932 & 22,991 & 23,281 & 24,518 & 26,264 & 28,280 & 30,279 & 32,407 \\
\hline Quota & 1,034 & & & & & & & \\
\hline GDP (in millions of U.S. dollars) & 166,513 & 178,723 & 184,151 & 194,270 & 205,926 & 219,322 & 234,695 & 251,146 \\
\hline Fund credit outstanding (in percent of GDP) & 3.0 & 5.2 & 6.2 & 5.3 & 3.6 & 1.4 & 0.2 & 0.0 \\
\hline Of which: SBA & 2.4 & 4.8 & 5.9 & 5.1 & 3.5 & 1.4 & 0.2 & 0.0 \\
\hline Gross international reserves (in millions of U.S. dollars) & 9,114 & 13,474 & 15,074 & 14,574 & 13,074 & 12,074 & 13,774 & 15,874 \\
\hline
\end{tabular}

Source: Fund staff projections.

1/ For 2008/09, debt service includes payments related to EFF. 
Table 11a. Pakistan: Access and Phasing Under the Proposed Augmented Stand-By Arrangement, 2008-10

\begin{tabular}{|c|c|c|c|c|c|c|c|c|c|c|}
\hline \multirow{2}{*}{ Review } & \multicolumn{2}{|c|}{ Date of availability } & \multicolumn{2}{|c|}{$\begin{array}{c}\text { Approved access and phasing } \\
\text { of purchases } \\
\end{array}$} & \multicolumn{2}{|c|}{ Proposed augmentation } & \multicolumn{2}{|c|}{$\begin{array}{c}\text { Proposed access and phasing } \\
\text { of purchases } \\
\end{array}$} & \multicolumn{2}{|c|}{ Of which: for budget financing } \\
\hline & Original & Revised & $\begin{array}{c}\text { In millions of } \\
\text { SDRs } \\
\end{array}$ & $\begin{array}{c}\text { In percent of } \\
\text { quota }\end{array}$ & $\begin{array}{c}\text { In millions of } \\
\text { SDRs }\end{array}$ & $\begin{array}{c}\text { In percent of } \\
\text { quota }\end{array}$ & $\begin{array}{c}\text { In millions of } \\
\text { SDRs }\end{array}$ & $\begin{array}{c}\text { In percent of } \\
\text { quota }\end{array}$ & $\begin{array}{c}\text { In millions of } \\
\text { SDRs } \\
\end{array}$ & $\begin{array}{c}\text { In percent of } \\
\text { quota } \\
\end{array}$ \\
\hline Board Approval & 24-Nov-08 & 24-Nov-08 & $2,067.400$ & 200.00 & $\ldots$ & & $2,067.400$ & 200.00 & $\ldots$ & \\
\hline First Review & 15-Mar-09 & 30-Mar-09 & 568.535 & 55.00 & $\ldots$ & $\ldots$ & 568.535 & 55.00 & $\ldots$ & \\
\hline Second Review & 15-Jun-09 & 07-Aug-09 & 568.540 & 55.00 & 198.160 & 19.17 & 766.700 & 74.17 & 475.600 & 46.01 \\
\hline Third Review & 15-Sep-09 & 30-Nov-09 & 434.150 & 42.00 & 332.550 & 32.17 & 766.700 & 74.17 & 237.750 & 23.00 \\
\hline Fourth Review & 15-Dec-09 & 28-Feb-10 & 434.150 & 42.00 & 332.550 & 32.17 & 766.700 & 74.17 & 237.750 & 23.00 \\
\hline Fifth Review & 15-Mar-10 & 31-May-10 & 434.150 & 42.00 & 332.550 & 32.17 & 766.700 & 74.17 & $\ldots$ & \\
\hline Sixth Review & 15-Jun-10 & 31-Aug-10 & 434.150 & 42.00 & 332.550 & 32.17 & 766.700 & 74.17 & $\ldots$ & .. \\
\hline Seventh Review & $15-S e p-10$ & $30-N o v-10$ & 227.425 & 22.00 & 539.040 & 52.15 & 766.465 & 74.15 & $\ldots$ & \\
\hline Total: & & & $5,168.500$ & 500.00 & 2067.400 & 200.00 & $7,235.900$ & 700.00 & 951.100 & 92.01 \\
\hline
\end{tabular}

Source: Fund staff.

1/The first review was completed on March 30 and the purchase was made on April 1.

Table 11b. Pakistan: Access and Phasing under the Proposed Augmented Stand-By Arrangement, 2008-10

\begin{tabular}{|c|c|c|c|c|}
\hline \multirow[t]{2}{*}{ Review } & \multirow[t]{2}{*}{ Availability Date } & \multirow[t]{2}{*}{ Action } & \multicolumn{2}{|c|}{ Purchase } \\
\hline & & & $\begin{array}{l}\text { In millions } \\
\text { of SDRs }\end{array}$ & $\begin{array}{c}\text { In percent } \\
\text { of quota }\end{array}$ \\
\hline & November 24, 2008 & Board approval of SBA & $2,067.400$ & 200.00 \\
\hline First Review & March 30, 2009 1/ & Observance of end-December 2008 performance criteria, completion of first review & 568.535 & 55.00 \\
\hline Second Review & August 7, 2009 & Observance of end-June 2009 performance criteria, completion of second review & 766.700 & 74.17 \\
\hline Third Review & November 30, 2009 & Observance of end-September 2009 performance criteria, completion of third review & 766.700 & 74.17 \\
\hline Fourth Review & February 28, 2010 & Observance of end-December 2009 performance criteria, completion of fourth review & 766.700 & 74.17 \\
\hline Fifth Review & May 31, 2010 & Observance of end-March 2010 performance criteria, completion of fifth review & 766.700 & 74.17 \\
\hline Sixth Review & August 31,2010 & Observance of end-June 2010 performance criteria, completion of sixth review & 766.700 & 74.17 \\
\hline Seventh Review & November 30, 2010 & Observance of end-September 2010 performance criteria, completion of seventh review & 766.465 & 74.15 \\
\hline Total & & & $7,235.900$ & 700.00 \\
\hline
\end{tabular}

Source: Fund staff.

1/The first review was completed on March 30 and the purchase was made on April 1. 
Table 12. Pakistan: Selected Vulnerability Indicators, 2007/08-2013/14

\begin{tabular}{|c|c|c|c|c|c|c|c|}
\hline & $2007 / 08$ & $\frac{2008 / 09}{\text { Est. }}$ & $2009 / 10$ & $2010 / 11$ & $\frac{2011 / 12}{\text { Projection }}$ & $2012 / 13$ & $2013 / 14$ \\
\hline \multicolumn{8}{|l|}{ Key economic and market indicators } \\
\hline Real GDP growth (factor cost, in percent) & 4.1 & 2.0 & 3.0 & 4.0 & 4.5 & 5.0 & 5.5 \\
\hline $\mathrm{CPI}$ inflation (period average, in percent) & 12.0 & 20.8 & 10.0 & 7.0 & 6.0 & 6.0 & 6.0 \\
\hline Short-term (ST) interest rate (in percent) & 11.5 & $\ldots$ & $\ldots$ & $\ldots$ & $\ldots$ & $\ldots$ & ... \\
\hline $\begin{array}{l}\text { Emerging market bond index (EMBI) secondary market } \\
\text { spread (basis points, end of period) }\end{array}$ & 687 & 1,039 & $\ldots$ & $\ldots$ & $\ldots$ & $\ldots$ & $\ldots$ \\
\hline Exchange rate PRs/US\$ (end of period) & 68.2 & 81.5 & $\ldots$ & $\ldots$ & $\ldots$ & $\ldots$ & $\ldots$ \\
\hline \multicolumn{8}{|l|}{ External sector } \\
\hline Exchange rate regime & \multicolumn{7}{|c|}{ Floating since January 2008} \\
\hline Current account balance (percent of GDP) & -8.3 & -5.1 & -4.7 & -5.3 & -5.0 & -4.3 & -3.4 \\
\hline Net FDI inflows (percent of GDP) & 3.2 & 2.1 & 1.7 & 1.9 & 2.1 & 2.2 & 2.3 \\
\hline Exports (percentage change of U.S. dollar value; GNFS) & 12.1 & -4.5 & 0.3 & 1.3 & 5.3 & 7.1 & 7.7 \\
\hline Real effective exchange rate (2000 $=100$, annual average) & 97.1 & $\ldots$ & $\ldots$ & $\ldots$ & $\ldots$ & $\ldots$ & $\ldots$ \\
\hline Gross international reserves (GIR) in billions of U.S. dollars & 8.6 & 9.1 & 13.5 & 15.1 & 14.6 & 13.1 & 12.1 \\
\hline GIR in percent of ST debt at remaining maturity (RM) 1/ & 441.5 & 328.7 & 615.5 & $1,088.3$ & $1,002.2$ & 828.6 & 736.9 \\
\hline GIR in percent of ST debt at RM and banks' foreign exchange (FX) deposits $1 /$ & 147.9 & 146.7 & 234.0 & 278.1 & 260.8 & 224.1 & 199.9 \\
\hline Total gross external debt (ED) in percent of GDP, of which: & 27.0 & 29.9 & 32.1 & 35.1 & 35.5 & 34.5 & 32.5 \\
\hline ST external debt (original maturity, in percent of total ED) & 1.8 & 1.6 & 0.9 & 0.3 & 0.3 & 0.3 & 0.3 \\
\hline ED of domestic private sector (in percent of total ED) & 6.5 & 6.4 & 6.0 & 5.7 & 5.6 & 5.5 & 5.4 \\
\hline ED to foreign official sector (in percent of total ED) & 93.5 & 93.6 & 94.0 & 94.3 & 94.4 & 94.5 & 94.6 \\
\hline Total gross external debt in percent of exports & 185.3 & 217.4 & 249.7 & 277.5 & 281.4 & 270.2 & 252.3 \\
\hline Gross external financing requirement (in billions of U.S. dollars) 2/ & 15.1 & 11.5 & 11.2 & 11.7 & 12.3 & 13.4 & 13.5 \\
\hline Public sector $3 /$ & \multicolumn{7}{|c|}{ (In percent of GDP) } \\
\hline Overall balance (including grants) & -7.3 & -5.0 & -4.2 & -3.8 & -3.0 & -2.8 & -2.6 \\
\hline Primary balance (including grants) & -2.5 & -0.2 & 0.2 & 0.0 & 0.4 & 0.3 & 0.1 \\
\hline Debt-stabilizing primary balance $4 /$ & -1.4 & -3.7 & -1.4 & -0.2 & -0.2 & -0.6 & -1.0 \\
\hline Gross PS financing requirement 5 / & 24.7 & 22.0 & 21.6 & 19.7 & 17.7 & 16.4 & 15.2 \\
\hline Public sector gross debt & 58.4 & 56.0 & 56.8 & 57.1 & 57.1 & 56.8 & 56.3 \\
\hline Public sector net debt $6 /$ & 53.8 & 51.3 & 52.2 & 52.5 & 52.5 & 52.1 & 51.6 \\
\hline \multicolumn{8}{|l|}{ Financial sector 71} \\
\hline Capital adequacy ratio (in percent) & 12.1 & $\ldots$ & $\ldots$ & $\ldots$ & $\ldots$ & $\ldots$ & ... \\
\hline Nonperforming loans (NPLs) in percent of total loans & 10.2 & $\ldots$ & $\ldots$ & $\ldots$ & $\ldots$ & $\ldots$ & ... \\
\hline Provisions in percent of NPLs & 75.0 & $\ldots$ & $\ldots$ & $\ldots$ & $\cdots$ & $\ldots$ & ... \\
\hline Return on average assets (in percent) & 1.7 & $\ldots$ & $\ldots$ & $\ldots$ & $\ldots$ & $\ldots$ & ... \\
\hline Return on equity (in percent) & 16.7 & $\ldots$ & $\ldots$ & $\ldots$ & $\ldots$ & $\ldots$ & ... \\
\hline FX deposits held by residents (in percent of total deposits) & 7.1 & 7.0 & 6.7 & $\ldots$ & $\ldots$ & $\ldots$ & $\ldots$ \\
\hline Government debt held by FS (percent of total FS assets) & 31.4 & 38.9 & 37.7 & $\ldots$ & $\ldots$ & $\ldots$ & $\ldots$ \\
\hline Credit to private sector (percent change) & 16.4 & 0.7 & 14.8 & $\ldots$ & $\ldots$ & $\ldots$ & ... \\
\hline \multicolumn{8}{|l|}{ Memorandum item: } \\
\hline Nominal GDP (in billions of U.S. dollars) & 164.6 & 166.5 & 178.7 & 184.2 & 194.3 & 205.9 & 219.3 \\
\hline
\end{tabular}

Sources: Pakistani authorities; and Fund staff estimates and projections.

$1 /$ Debt at remaining maturity is defined as maturing short-, medium-, and long-term external official debt.

2/ Current account deficit plus amortization of external debt.

3/ Public sector covers general (consolidated) government.

4/ Based on the end of period debt stock in year $\mathrm{t}-1$, and the baseline assumptions for the relevant variables (i.e., growth, interest rates

inflation, exchange rates) in year $\mathrm{t}$.

$5 /$ Overall balance plus debt amortization

6/ Net debt is defined as gross debt minus government deposits with the banking system.

7/ Financial sector includes all commercial and specialized banks; for government debt also includes nonbanks, but excludes State Bank of Pakistan. 
Table 13. Pakistan: Financial Soundness Indicators for the Banking System

(December 2004-March 2009)

\begin{tabular}{|c|c|c|c|c|c|c|c|}
\hline & $\begin{array}{c}2004 \\
\text { Dec. }\end{array}$ & $\begin{array}{l}2005 \\
\text { Dec. }\end{array}$ & $\begin{array}{c}2006 \\
\text { Dec. }\end{array}$ & $\begin{array}{c}2007 \\
\text { Dec. }\end{array}$ & $\begin{array}{c}2008 \\
\text { Mar. }\end{array}$ & $\begin{array}{c}2008 \\
\text { Dec. }\end{array}$ & $\begin{array}{r}2009 \\
\text { Mar. }\end{array}$ \\
\hline \multicolumn{8}{|l|}{ Capital adequacy } \\
\hline Regulatory capital to risk-weighted assets & 10.5 & 11.3 & 12.7 & 13.2 & 13.0 & 12.2 & 12.9 \\
\hline Tier I capital to risk-weighted assets & 7.6 & 8.3 & 10.0 & 10.5 & 10.5 & 10.2 & 10.8 \\
\hline Capital to total assets & 6.7 & 7.9 & 9.4 & 10.5 & 10.4 & 10.4 & 10.3 \\
\hline \multicolumn{8}{|l|}{ Asset composition and quality } \\
\hline Nonperforming loans (NPLs) to gross loans & 11.6 & 8.3 & 6.9 & 7.2 & 7.7 & 9.1 & 11.5 \\
\hline Provisions to NPLs & 70.4 & 76.7 & 77.8 & 85.1 & 84.1 & 74.7 & 69.2 \\
\hline NPLs net of provisions to capital & 29.2 & 14.3 & 9.7 & 5.6 & 6.8 & 13.6 & 19.6 \\
\hline \multicolumn{8}{|l|}{ Earnings and profitability } \\
\hline Return on assets (after tax) & 1.2 & 1.9 & 2.1 & 1.5 & 1.4 & 1.2 & 1.1 \\
\hline Return on equity (after tax) & 20.3 & 25.8 & 23.8 & 15.5 & 13.2 & 11.3 & 10.7 \\
\hline Net interest income to gross income & 62.8 & 72.0 & 70.9 & 68.3 & 69.1 & 70.7 & 75.8 \\
\hline Noninterest expenses to gross income & 52.0 & 41.5 & 40.3 & 43.0 & 44.6 & 49.1 & 49.9 \\
\hline \multicolumn{8}{|l|}{ Liquidity } \\
\hline Liquid assets to total assets & 36.6 & 33.7 & 31.9 & 33.6 & 31.0 & 27.5 & 30.5 \\
\hline Liquid assets to total deposits & 46.5 & 43.5 & 42.7 & 45.1 & 41.7 & 36.9 & 41.5 \\
\hline
\end{tabular}

Source: State Bank of Pakistan. 


\section{ApPendix. Pakistan: Debt Sustainability Analysis}

Figure 1. Pakistan: External Debt Sustainability-Bound Tests 1/

(External debt in percent of GDP)
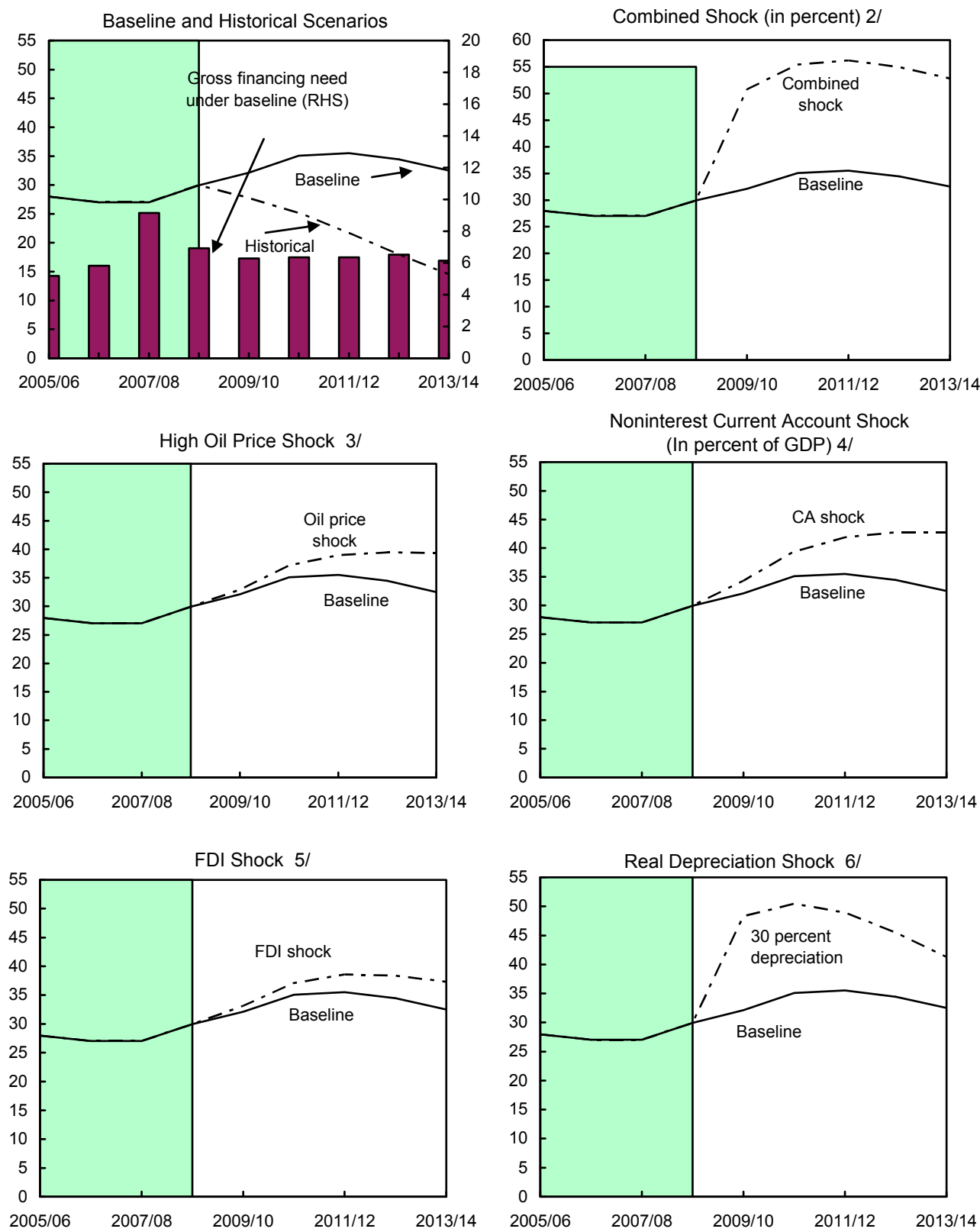

Sources: Historical data from Pakistani authorities; and Fund staff estimates and projections,

1/ Shaded areas represent actual data. Individual shocks are permanent one-half standard deviation shocks. Figures in the boxes represent average projections for the respective variables in the baseline and scenario being presented. Ten-year historical average for the variable is also shown.

2/ Combined impact of shocks to growth, current account, FDI, and real depreciation.

$3 /$ Oil prices are higher by $\$ 10$ for the projection period relative to the baseline.

4/ Current account deficit higher than the baseline scenario by half of the ten-year standard deviation.

$5 /$ Net flows of foreign direct investment are 50 percent lower than in the baseline scenario.

6 / One-time real depreciation of 30 percent occurs in 2009/10. 
Table 1. Pakistan: External Debt Sustainability Framework, 2004/05-2013/14

(In percent of GDP, unless otherwise indicated)

\begin{tabular}{|c|c|c|c|c|c|c|c|c|c|c|c|}
\hline \multirow[b]{3}{*}{ Baseline: External debt } & \multicolumn{4}{|c|}{ Actual } & \multicolumn{6}{|c|}{ Projections } & \multirow{3}{*}{$\begin{array}{c}\begin{array}{c}\text { Debt-stabilizing } \\
\text { non-interest current } \\
\text { account } 71\end{array} \\
-4.0\end{array}$} \\
\hline & \multirow{2}{*}{$\begin{array}{r}2004 / 05 \\
31.1\end{array}$} & \multirow{2}{*}{$\begin{array}{r}2005 / 06 \\
28.0\end{array}$} & \multirow{2}{*}{$\begin{array}{r}2006 / 07 \\
27.0\end{array}$} & \multirow{2}{*}{$\begin{array}{r}2007 / 08 \\
27.0\end{array}$} & \multirow{2}{*}{$\begin{array}{r}2008 / 09 \\
29.9\end{array}$} & \multirow{2}{*}{$\begin{array}{r}2009 / 10 \\
32.1\end{array}$} & \multirow{2}{*}{$\begin{array}{r}2010 / 11 \\
35.1\end{array}$} & \multirow{2}{*}{$\begin{array}{r}2011 / 12 \\
35.5\end{array}$} & \multirow{2}{*}{$\begin{array}{r}2012 / 13 \\
34.5\end{array}$} & \multirow{2}{*}{$\begin{array}{r}2013 / 14 \\
32.5\end{array}$} & \\
\hline & & & & & & & & & & & \\
\hline Change in external debt & -3.0 & -3.1 & -0.9 & 0.0 & 2.9 & 2.2 & 3.0 & 0.4 & -1.1 & -1.9 & \\
\hline Identified external debt-creating flows $(4+8+9)$ & -3.7 & -3.5 & -3.5 & 1.5 & 2.9 & 2.2 & 1.9 & 1.1 & 0.0 & -1.1 & \\
\hline Current account deficit, excluding interest payments & 0.5 & 2.9 & 3.8 & 7.0 & 4.0 & 3.7 & 4.4 & 4.1 & 3.4 & 2.6 & \\
\hline Deficit in balance of goods and services & 7.1 & 10.1 & 9.7 & 12.9 & 9.1 & 7.9 & 8.6 & 8.4 & 7.9 & 7.4 & \\
\hline Exports & 16.2 & 15.9 & 15.0 & 14.6 & 13.8 & 12.9 & 12.6 & 12.6 & 12.8 & 12.9 & \\
\hline Imports & 23.4 & 26.0 & 24.6 & 27.5 & 22.9 & 20.8 & 21.2 & 21.1 & 20.7 & 20.3 & \\
\hline Net non-debt-creating capital inflows (negative) & -1.5 & -3.1 & -5.2 & -3.3 & -1.7 & -1.7 & -2.2 & -2.5 & -2.7 & -2.7 & \\
\hline Automatic debt dynamics $1 /$ & -2.6 & -3.4 & -2.1 & -2.2 & 0.6 & 0.2 & -0.3 & -0.5 & -0.8 & -1.0 & \\
\hline Contribution from nominal interest rate & 0.9 & 1.0 & 1.0 & 1.3 & 1.1 & 1.0 & 0.9 & 1.0 & 0.9 & 0.8 & \\
\hline Contribution from real GDP growth & -2.3 & -1.6 & -1.4 & -0.5 & -0.5 & -0.8 & -1.2 & -1.5 & -1.7 & -1.8 & \\
\hline Contribution from price and exchange rate changes $2 /$ & -1.2 & -2.7 & -1.7 & -3.0 & & & $\ldots$ & & & & \\
\hline Residual, incl. change in gross foreign assets $(2-3) 3 /$ & 0.7 & 0.4 & 2.5 & -1.5 & 0.0 & 0.0 & 1.1 & -0.6 & -1.1 & -0.9 & \\
\hline External debt-to-exports ratio (in percent) & 191.2 & 175.5 & 180.7 & 185.3 & 217.4 & 249.7 & 277.5 & 281.4 & 270.2 & 252.3 & \\
\hline Gross external financing need (in billions of US dollars) 4 / & 3.5 & 6.6 & 8.3 & 15.1 & 11.5 & 11.2 & 11.7 & 12.3 & 13.4 & 13.5 & \\
\hline In percent of GDP & 3.2 & 5.2 & 5.8 & 9.2 & 6.9 & 6.3 & 6.3 & 6.4 & 6.5 & 6.1 & \\
\hline Scenario with key variables at their historical averages $5 /$ & & $\ldots$ & $\ldots$ & & 29.9 & 27.8 & 25.2 & 21.7 & 17.9 & 14.5 & -2.3 \\
\hline \multicolumn{12}{|l|}{ Key Macroeconomic Assumptions Underlying Baseline } \\
\hline Real GDP growth (in percent) & 7.7 & 6.1 & 5.6 & 2.0 & 2.0 & 3.0 & 4.0 & 4.5 & 5.0 & 5.5 & \\
\hline GDP deflator in US dollars (change in percent) & 3.8 & 9.6 & 6.3 & 12.6 & -0.8 & 4.2 & -0.9 & 1.0 & 1.0 & 1.0 & \\
\hline Nominal external interest rate (in percent) & 3.1 & 3.7 & 4.0 & 5.6 & 4.3 & 3.7 & 3.0 & 2.9 & 2.7 & 2.6 & \\
\hline Growth of exports of goods and services (U.S. dollar terms, in percent) $6 /$ & 17.9 & 14.2 & 5.4 & 12.1 & -4.5 & 0.3 & 1.3 & 5.3 & 7.1 & 7.7 & \\
\hline Growth of imports of goods and services (U.S. dollar terms, in percent) $6 /$ & 44.7 & 29.6 & 6.3 & 28.3 & -16.0 & -2.5 & 5.4 & 4.5 & 4.2 & 4.6 & \\
\hline Current account balance, excluding interest payments & -0.5 & -2.9 & -3.8 & -7.0 & -4.0 & -3.7 & -4.4 & -4.1 & -3.4 & -2.6 & \\
\hline Net non-debt-creating capital inflows & 1.5 & 3.1 & 5.2 & 3.3 & 1.7 & 1.7 & 2.2 & 2.5 & 2.7 & 2.7 & \\
\hline
\end{tabular}

Sources: Pakistani authorities; and Fund staff estimates and projections.

1/ Derived as $[r-g-\rho(1+g)+e a(1+r)](1+g+\rho+g \rho)$ times previous period debt stock, with $r=$ nominal effective interest rate on external debt; $\rho=$ change in domestic GDP deflator in U.S. dollar terms, $g=$ real GDP growth rate, $e=$ nominal appreciation (increase in dollar value of domestic currency), and $a=$ share of domestic-currency denominated debt in total external debt.

$2 /$ The contribution from price and exchange rate changes is defined as $[-\rho(1+g)+e a(1+r)] /(1+g+\rho+g \rho)$ times previous period debt stock. rho increases with an appreciating domestic currency $(\mathrm{e}>0)$ and rising inflation (based on GDP deflator).

/ For projection, line includes the impact of price and exchange rate changes.

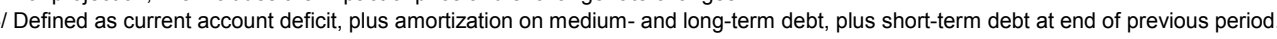

The key variables include real GDP growth, nom

7/ Long-run, constant balance that stabilizes the debt ratio assuming that key variables (real GDP growth, nominal interest rate, dollar deflator growth, and non-debt inflows in percent of GDP) remain at their levels of the last projection year. 
Figure 2. Pakistan: Public Debt Sustainability: Bound Tests 1/ (Public debt in percent of GDP)

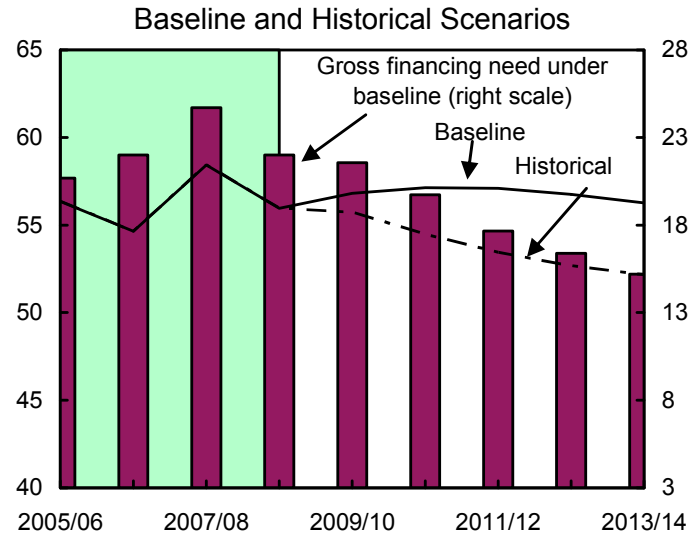

Growth Shock (in percent per year)

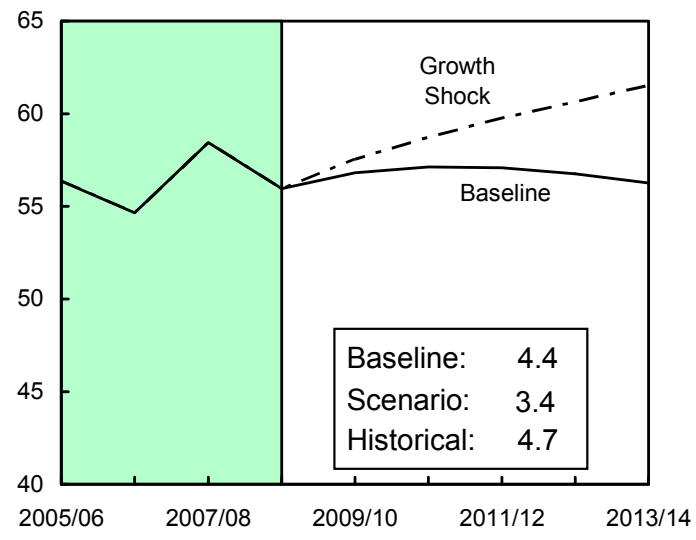

Combined Shock 2/

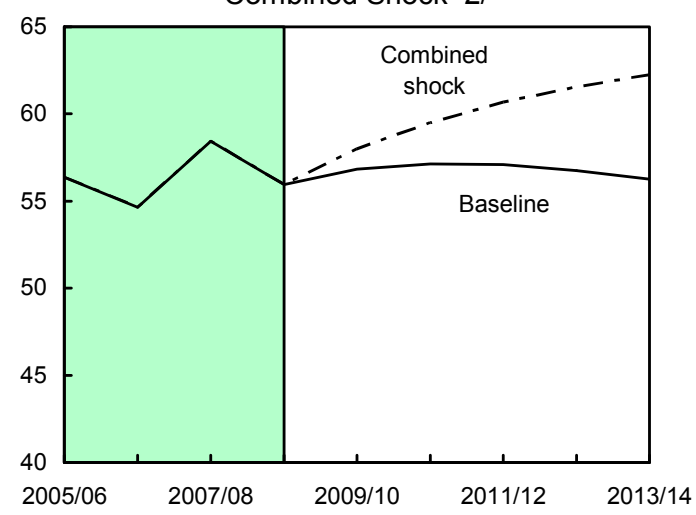

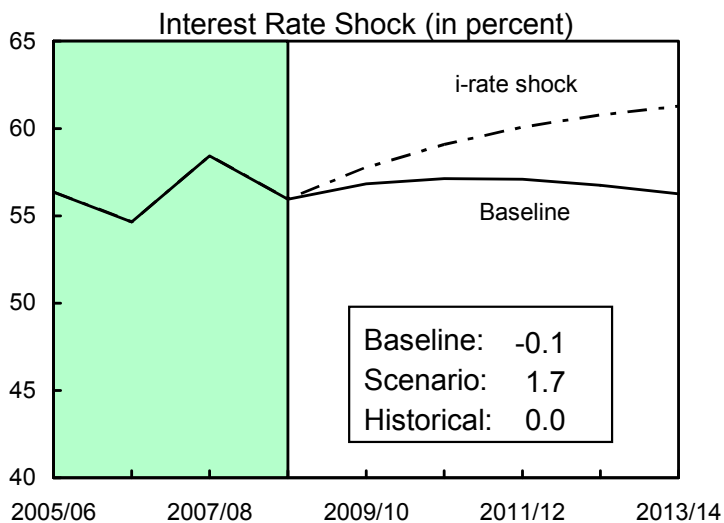

Primary Balance Shock (in percent of GDP) and No policy Change Scenario (constant primary balance)

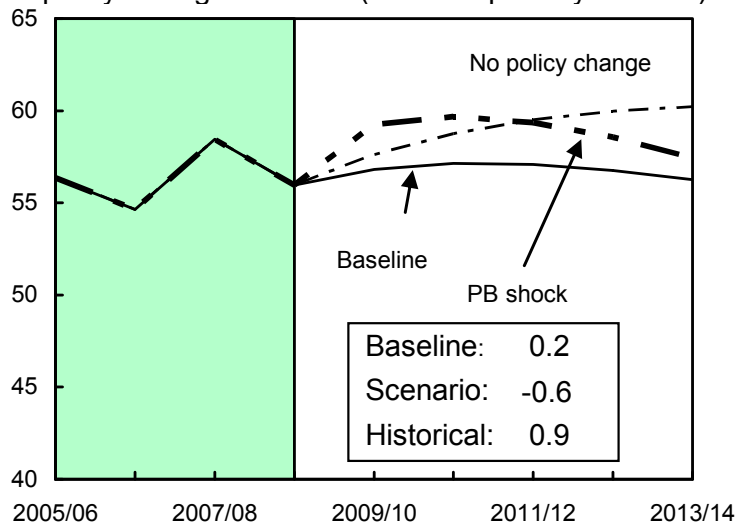

Real Depreciation and Contingent Liabilities Shocks

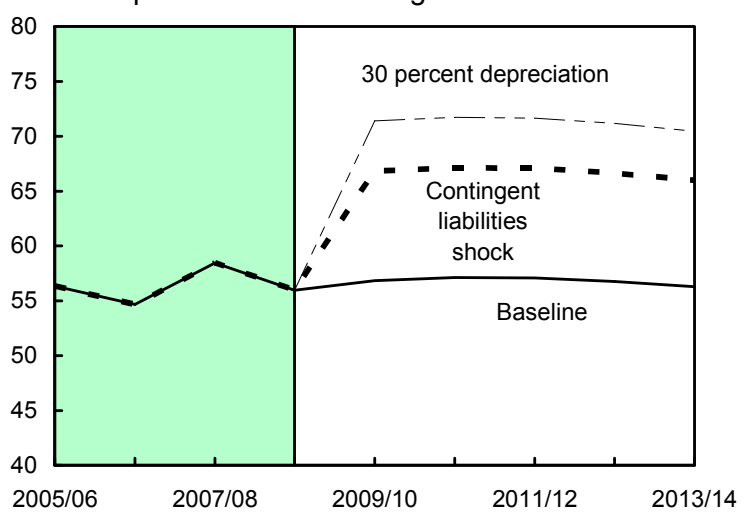

Source: Fund staff estimates and projections.

1/ Shaded areas represent actual data. Individual shocks are permanent one-half standard deviation shocks. Figures in the boxes represent average projections for the respective variables in the baseline and scenario being presented. Ten-year historical average for the variable is also shown.

2/ Permanent $1 / 4$ standard deviation shocks applied to real interest rate, growth rate, and primary balance. $3 /$ One-time real depreciation of 30 percent and 10 percent of GDP shock to contingent liabilities occur in 2009/10, with real depreciation defined as nominal depreciation (measured by percentage fall in dollar value of local currency) minus domestic inflation (based on GDP deflator). 
Table 2. Pakistan: Public Sector Debt Sustainability Framework, 2004/05-2013/14

(In percent of GDP, unless otherwise indicated)

\begin{tabular}{|c|c|c|c|c|c|c|c|c|c|c|c|}
\hline \multirow{3}{*}{$\begin{array}{l}\text { Baseline: Public sector debt } 1 / \\
\text { Of which: Foreign-currency denominated }\end{array}$} & \multicolumn{4}{|c|}{$\begin{array}{c}\text { Actual } \\
\text { 2004/05 2005/06 2006/07 2007/08 }\end{array}$} & \multicolumn{6}{|c|}{$\begin{array}{c}\text { Projections } \\
\text { 2008/09 2009/10 2010/11 2011/12 2012/13 2013/14 }\end{array}$} & \multirow{2}{*}{$\begin{array}{c}\begin{array}{r}\text { Debt-stabilizing } \\
\text { primary balance 9/ }\end{array} \\
-0.4\end{array}$} \\
\hline & 62.0 & 56.4 & 54.6 & 58.4 & 56.0 & 56.8 & 57.1 & 57.1 & 56.8 & 56.3 & \\
\hline & 28.9 & 25.9 & 24.7 & 26.7 & 26.0 & 25.6 & 27.6 & 29.8 & 31.5 & 32.9 & \\
\hline Change in public sector debt & -6.3 & -5.6 & -1.7 & 3.8 & -2.5 & 0.9 & 0.3 & 0.0 & -0.3 & -0.5 & \\
\hline Identified debt-creating flows $(4+7+12)$ & -4.3 & -5.0 & -2.6 & 1.1 & -2.4 & -2.0 & -1.6 & -2.2 & -2.7 & -3.0 & \\
\hline Primary deficit & -0.2 & 0.6 & -0.2 & 2.5 & 0.2 & -0.2 & 0.0 & -0.4 & -0.3 & -0.1 & \\
\hline Revenue and grants & 14.1 & 14.7 & 15.3 & 14.9 & 14.4 & 15.2 & 15.4 & 16.0 & 16.3 & 16.6 & \\
\hline Primary (noninterest) expenditure & 14.0 & 15.3 & 15.1 & 17.4 & 14.6 & 15.0 & 15.5 & 15.6 & 16.0 & 16.4 & \\
\hline Automatic debt dynamics $2 /$ & -5.0 & -5.8 & -2.4 & -1.1 & -3.6 & -2.2 & -2.0 & -2.1 & -2.7 & -3.3 & \\
\hline Contribution from interest rate/growth differential $3 /$ & -5.8 & -6.0 & -2.6 & -3.8 & -7.7 & -2.2 & -2.0 & -2.1 & -2.7 & -3.3 & \\
\hline Of which: Contribution from real interest rate & -1.2 & -2.8 & 0.2 & -2.9 & -6.8 & -0.7 & 0.1 & 0.2 & -0.2 & -0.5 & \\
\hline Of which: Contribution from real GDP growth & -4.5 & -3.2 & -2.8 & -0.9 & -0.9 & -1.5 & -2.0 & -2.3 & -2.6 & -2.8 & \\
\hline Contribution from exchange rate depreciation $4 /$ & 0.8 & 0.2 & 0.1 & 2.7 & 4.2 & $\ldots$ & & $\ldots$ & & & \\
\hline Other identified debt-creating flows & 0.9 & 0.2 & 0.1 & -0.3 & 1.0 & 0.4 & 0.3 & 0.3 & 0.3 & 0.4 & \\
\hline Privatization receipts (negative) & -0.4 & -1.3 & -0.8 & 0.0 & 0.0 & -0.1 & -0.2 & -0.2 & -0.1 & -0.1 & \\
\hline Recognition of implicit or contingent liabilities & 0.0 & 0.0 & 0.0 & 0.0 & 0.0 & 0.0 & 0.0 & 0.0 & 0.0 & 0.0 & \\
\hline Build-up of bank deposits & 1.3 & 1.5 & 0.9 & -0.3 & 1.0 & 0.5 & 0.5 & 0.5 & 0.5 & 0.5 & \\
\hline Residual, including other asset changes (2-3) 5/ & -2.0 & -0.6 & 0.9 & 2.7 & -0.1 & 2.9 & 1.9 & 2.2 & 2.4 & 2.5 & \\
\hline Public sector debt-to-revenue ratio $1 /$ & 438.2 & 383.3 & 357.1 & 392.8 & 388.2 & 374.6 & 370.3 & 357.6 & 348.7 & 339.2 & \\
\hline Gross financing need 6 / & 19.0 & 20.7 & 22.0 & 24.7 & 22.0 & 21.6 & 19.7 & 17.7 & 16.4 & 15.2 & \\
\hline In billions of U.S. dollars & 20.8 & 26.4 & 31.5 & 40.6 & 36.6 & 38.5 & 36.3 & 34.3 & 33.8 & 33.3 & \\
\hline Scenario with key variables at their historical averages $7 /$ & & & $\ldots$ & $\ldots$ & 56.0 & 55.7 & 54.5 & 53.5 & 52.7 & 52.2 & 0.4 \\
\hline Scenario with no policy change (constant primary balance) in 2009/10-2013/14 & $\ldots$ & $\ldots$ & $\ldots$ & $\ldots$ & 56.0 & 59.3 & 59.7 & 59.4 & 58.6 & 57.4 & -1.4 \\
\hline \multicolumn{12}{|l|}{ Key Macroeconomic and Fiscal Assumptions Underlying Baseline } \\
\hline Real GDP growth at market prices (in percent) & 7.7 & 6.1 & 5.6 & 2.0 & 2.0 & 3.0 & 4.0 & 4.5 & 5.0 & 5.5 & \\
\hline Average nominal interest rate on public debt (in percent) $8 /$ & 5.5 & 5.9 & 8.6 & 10.3 & 10.4 & 8.9 & 7.5 & 6.6 & 6.0 & 5.3 & \\
\hline Average real interest rate (nominal rate minus change in GDP deflator, in percent) & -1.6 & -4.6 & 0.9 & -5.9 & -14.4 & -1.1 & 0.5 & 0.6 & 0.0 & -0.7 & \\
\hline Nominal appreciation (increase in U.S. dollar value of local currency, in percent) & -2.5 & -0.9 & -0.5 & -11.4 & & & & & & & \\
\hline Inflation rate (GDP deflator, in percent) & 7.0 & 10.5 & 7.7 & 16.2 & 24.9 & 10.0 & 7.0 & 6.0 & 6.0 & 6.0 & \\
\hline Growth of real primary spending (deflated by GDP deflator, in percent) & 16.5 & 16.2 & 4.2 & 17.8 & -14.5 & 5.6 & 7.5 & 5.4 & 7.7 & 8.5 & \\
\hline Primary deficit & -0.2 & 0.6 & -0.2 & 2.5 & 0.2 & -0.2 & 0.0 & -0.4 & -0.3 & -0.1 & \\
\hline
\end{tabular}

Sources: Pakistani authorities; and Fund staff estimates and projections.

1/ General government gross debt; excludes obligations to the IMF, external military debt, commercial debt, short-term loans, and debt from public enterprises.

2/ Derived as $[(r-p(1+g)-g+a e(1+r)] /(1+g+p+g p))$ times previous period debt ratio, with $r=$ interest rate; $p=$ growth rate of GDP deflator; $g=$ real GDP growth rate; $a=$ share of foreign-currency denominated debt; and $\mathrm{e}=$ nominal exchange rate depreciation (measured by increase in local currency value of U.S. dollar).

$3 /$ The real interest rate contribution is derived from the denominator in footnote $2 /$ as $r-\pi(1+g)$ and the real growth contribution as -g.

$4 /$ The exchange rate contribution is derived from the numerator in footnote $2 /$ as ae $(1+r)$.

5/ For projections, this line includes exchange rate changes. For 2009/10, it reflects, inter alia, the assumption by the government of electricity sector debt of Rs. 277 billion (1.9 percent of GDP). 6/ Defined as public sector deficit, plus amortization of medium and long-term public sector debt, plus short-term debt at end of previous period.

7/ The key variables include real GDP growth; real interest rate; and primary balance in percent of GDP.

8/ Derived as nominal interest expenditure divided by previous period debt stock.

9/ Assumes that key variables (real GDP growth, real interest rate, and other identified debt-creating flows) remain at the level of the last projection year. 


\section{Attachment I. Pakistan: Letter of Intent}

July 27, 2009

Mr. Dominique Strauss-Kahn

Managing Director

International Monetary Fund

Washington, D.C. 20431

Dear Mr. Strauss-Kahn:

The Pakistani authorities held discussions with Fund staff for the second review under the Stand-By Arrangement (SBA). Based on these discussions, we have updated our economic program for 2009/10, as reflected in the attached Second Supplementary Memorandum of Economic and Financial Policies (SSMEFP). In support of our policies, the government requests that the Executive Board of the Fund consider an augmentation of the SBA in an amount of SDR 2,067.4 billion (200 percent of Pakistan's quota), complete the second review, and approve the third purchase under the SBA of SDR 766.7 million.

Our economic stabilization program has surmounted obstacles, but we face difficult challenges. The contracting world economy and higher oil prices are affecting Pakistan's domestic economy, trade, and external financing. The program's objectives have been modified in light of the slowdown in economic activity and our authorities believe that the policies set out in the attached SSMEFP are adequate to achieve these objectives. However, our authorities stand ready to take any additional measures deemed appropriate for this purpose, and will consult with the Fund on the adoption of these measures in accordance with Fund policies on such consultations. Our authorities will provide the Fund with the information it may request to monitor progress in implementing its economic and financial policies.

We request waivers of nonobservance for the two end-June 2009 structural performance criteria on submission to parliament of legislative amendments to (i) enhance the effectiveness of the State Bank of Pakistan in banking supervision; and to (ii) harmonize the income tax and sales tax laws and reduce exemptions for both taxes. We plan to submit the amendments to Banking Companies Ordinance to enhance SBP's enforcement powers in banking supervision by end-August, 2009, and the amendments to harmonize income and sales tax laws by end-September 2009. Both actions will be structural benchmarks for the third SBA review. Tax exemptions will be reduced with the introduction of VAT, which is scheduled to become effective on July 1, 2010.

We also request a waiver of nonobservance for the end-June quantitative performance criterion on the fiscal deficit. Although final data for the last quarter of 2008/09 are not yet available, preliminary information indicates that we missed the end-June 2009 quantitative performance criterion on the fiscal deficit by 0.9 percent of GDP, on account of securityrelated expenditures and unexpected borrowing by provinces. 
In order to accommodate the delay in the completion of the second review we request an extension of the present SBA through end-2010 and the rephasing of purchases with an additional test date for end-September 2010.

We consent to the publication of this letter, the attached SSMEFP, the revised Technical Memorandum of Understanding, and the related staff report.

Sincerely yours,

$/ \mathrm{s} /$

Shaukat Tarin

Advisor to the Prime Minister on Finance, Revenue, Economic Affairs and Statistics
$/ \mathrm{s} /$

Syed Salim Raza

Governor

State Bank of Pakistan

Attachments

Second Supplementary Memorandum of Economic and Financial Policies

Technical Memorandum of Understanding 


\section{Attachment II. Pakistan: Second Supplementary Memorandum on ECONOMIC AND FInANCIAL POLICIES FOR 2009/10}

July 29, 2009

\section{INTRODUCTION}

1. This memorandum supplements our Memorandum on Economic and Financial Policies (MEFP) for 2008/09-09/10 of November 20, 2008 and the first supplementary memorandum of March 16, 2009. It describes policy implementation to date and lays out additional policies agreed in the context of the second review under the Stand-By Arrangement (SBA).

\section{RECENT ECONOMIC DEVELOPMENTS}

2. Our stabilization program has surmounted major security and external difficulties in recent months. Headline inflation declined from 25 percent in October 2008 to 13.1 percent in June 2009, the rupee has remained stable at around Rs. 80 per U.S. dollar, gross foreign exchange reserves reached $\$ 9.1$ billion as of June 30, 2009, and the market's demand for Treasury bills has remained strong. All quantitative performance criteria through end-March 2009 were observed (Table 1). Preliminary data for end-June 2009 indicate that the quantitative performance criteria for the net foreign and domestic assets of the State Bank of Pakistan (SBP) and net government borrowing from the SBP were met, while the budget deficit target was exceeded by about 0.9 percent of GDP, on account of lower-thanprogrammed tax revenues and higher provincial expenditure, as well as security- and IDPrelated spending. Implementation of structural reforms suffered from delays during AprilJune 2009, but we are determined to press ahead with these reforms in the next few months.

3. The ongoing global economic crisis, negative security developments, and continued electricity shortages are slowing Pakistan's growth. The real GDP of Pakistan's main trading partners is projected to contract by almost 3 percent on average in 2009, depressing the demand for Pakistan's manufacturing exports. Private capital inflows have declined, and there are significant downside risks for workers' remittances. Overall economic activity has weakened, despite growth in agriculture, and real GDP growth in 2008/09 is now estimated at 2 percent. Due to upward pressure on food prices, headline inflation has declined more slowly than expected. Nevertheless, it declined to 13.1 percent year-on-year in June 2009.

4. Pressures on the budget had intensified. Declining imports and slowing domestic demand have resulted in lower-than-envisaged tax revenue, while expenditures have increased with larger security- and internally displaced persons (IDP)-related outlays and an unexpected surge in provincial spending. In particular, Federal Board of Revenue (FBR) revenues in 2008/09 fell short of the projection prepared at the time of the first review by 1.2 percentage points of GDP. This shortfall, however, has been offset in part by receipts from the Petroleum Development Levy (PDL), higher-than-expected nontax revenues, and 
expenditure restraint, especially with respect to development spending. However, owing to the need to step up security-related spending, it was not possible to offset fully the shortfall in revenue and the deficit target was exceeded. Disbursements of multilateral program financing have been lower than projected.

5. Increased market confidence and declining inflation provided an opportunity for the SBP to ease somewhat the monetary policy stance. On April 21, 2009, the SBP policy interest rate was lowered by 100 basis points, to 14 percent.

6. The donor conference that took place in Tokyo in April was successful. Pakistan received $\$ 5.7$ billion in new pledges over three years, which exceeded expectations. Most of the additional resources will be available for project financing - especially for social spending and IDP support - and a small portion for balance-of-payments assistance. Support group meetings are ongoing, and the details of donors' assistance are likely to be finalized by end-September 2009. This will provide an indication of the nature and timing of disbursements.

\section{POLICIES FOR 2009/10}

7. We believe that maintaining macroeconomic stability is essential for sustainable growth and the development of Pakistan's economy. We see the following main policy challenges for 2009/10: consolidating macroeconomic stability; mitigating the effects of the global economic crisis, in particular on exports and manufacturing; implementing tax policy and administration reforms; and managing the impact on the budget of security outlays.

8. We remain committed to implementing our stabilization and reform policies. The donor support pledged at the Tokyo conference provides scope for additional social expenditures and growth-oriented development spending. We will work with donors to ensure that the temporary fiscal easing envisaged for 2009/10 is supported by timely disbursements of foreign assistance and remains consistent with macroeconomic stabilization. We are mindful that the additional external support is temporary and will seek to ensure that it is used effectively and does not undermine our objective of significantly strengthening the fiscal position over the medium term. Accordingly, we remain committed to the strong tax reform efforts envisaged in the original program.

\section{We are requesting an augmentation of access under the Fund $S B A$ to meet the} larger-than-expected balance of payments needs and address the increased risks stemming from the global financial crisis. The augmentation will be used mostly to strengthen the SBP's international reserves and improve its ability to manage downside risks to the external position. About 45 percent of the higher access will be used for budget financing if disbursements of foreign assistance fall short of projected amounts. 
10. The program for $2009 / 10$ is built around the following revised economic parameters: real GDP growth of 3 percent; consumer price inflation (year-on-year) of 9 percent at year's end; and a current account deficit of $43 / 4$ percent of GDP.

\section{A. Fiscal Policy}

11. With the economy slowing and increased availability of external financing, some relaxation of the fiscal stance in 2009/10 is feasible and desirable. The additional donor support creates an opportunity for containing the slowdown in economic growth while allowing for a perceptible increase in social, infrastructure, and security expenditures, including support for IDPs. To avoid increasing medium-term risks, we consider the Tokyo package as a bridge until tax reforms (notably, the introduction of the VAT in 2010/11) bear their full fruit. Assuming about 1.1 percent of GDP in additional disbursements from the Tokyo pledges in 2009/10, the deficit target will be raised to 4.6 percent of GDP, compared to the original program target of 3.4 percent of GDP.

12. The budget deficit of 4.9 percent of GDP approved by Parliament is consistent with the program deficit target of 4.6 percent of GDP. The budget deficit target (excluding grants) would only be increased above 4.6 percent of GDP if additional IDP-related grants become available and are disbursed through the budget. ${ }^{1}$ If IDP-related grants are not forthcoming, the fiscal space for higher IDP-related spending will be created through additional revenue measures and or by cutting non-IDP development spending and other nonessential outlays.

13. To ensure fiscal discipline and the achievement of the deficit target of the general government, borrowing by the provinces will be strictly limited to the agreed level of Ways and Means advances. Most notably, the provinces will not be allowed to exceed the overdraft from the SBP beyond their agreed Ways and Means limits. The federal government, in consultation with provincial governments, will introduce, effective August 1, 2009, a binding Ways and Means ceiling of six weeks of the provincial wage bill. Moreover, agreements will be entered into by end-September 2009 between the provinces, the Ministry of Finance, and the SBP on a schedule of reduction of the overdraft stock.

14. An increase in the tax revenue-to-GDP ratio of $3 \frac{1}{2}$ percentage points over five years remains a key pillar of our medium-term fiscal strategy. This revenue effort, to be achieved through ambitious tax policy and administration reforms, will be essential to reduce macroeconomic vulnerability and scale-up infrastructure, security, and social spending in a sustainable manner. The temporary nature of the additional resources pledged in Tokyo renders perseverance in our revenue efforts even more important.

\footnotetext{
${ }^{1}$ The existing adjustor in the program accommodates additional budgetary grant-financed spending on IDPs. There are no limits on IDP grant-financed outlays by NGOs or the UN.
} 
15. The budget approved by Parliament includes the following tax policy measures that are expected to yield an increase in the tax revenue-to-GDP ratio of 0.4 percentage points in 2009/10:

- Introduction of a specific federal petroleum levy along with automatic pass-through to retail prices of changes in world oil prices;

- Introduction of a federal excise tax in VAT mode on selected services (e.g., banking services, insurance);

- Enhancement of the capital value tax (a transaction tax) on real estate transactions;

- Increase in the rate for the withholding tax on imports from 2 percent to 3 percent for imports used by industry and to 4 percent for other imports.

- Increase in the excise tax on cigarettes.

- Changes in the computation of the formula for the advance tax payable by the corporate sector.

16. The main pillar of our medium-term fiscal strategy is the introduction of a broadbased VAT on July 1, 2010, covering all goods and services. This takes account of the recommendations made at the Lahore Tax Policy Conference in December 2008. A project team was established and a project leader appointed on July 2, 2009. To ensure active and comprehensive stakeholder participation, we will begin the process of consultation on the VAT with a seminar in September, with the participation at the highest level of members of the federal and provincial governments, and supported by the IMF, the World Bank, and the Asian Development Bank (ADB). This seminar will present proposals and options for the introduction and implementation of the VAT. A detailed time-bound action plan for the introduction of the VAT will be prepared by end-September 2009. We will submit the VAT law to Parliament by end-December 2009 (structural benchmark). We are receiving Fund assistance on policy aspects for introducing the VAT.

17. Our tax policy reforms will be complemented with reinvigorated efforts in the area of tax administration. We have reached understandings with World Bank staff on the organization of a functionally structured tax administration which will integrate the responsibilities for domestic taxes, including the sales, income, and excise taxes, in one occupational group within the FBR. This functional organization is designed both to increase the effectiveness of tax administration and facilitate the introduction of the VAT. The following specific actions have already been taken or will be adopted in the coming months:

- To ensure swift implementation of this reform, the FBR Chairman issued an Executive Order on June 30, 2009, to put in place the new FBR management structure. We will complete the functional reorganization of tax administration by end-September, 2009. To this end, by September 15, 2009, the government will approve regulations (structural benchmark) to (i) form new occupational groups 
within the FBR; and (ii) revise the structures of Regional Taxpayer Offices (RTOs) and Large Taxpayer Units (LTUs). Also, all relevant laws and rules will be amended.

- We will submit to Parliament by end-September 2009 legislative amendments to harmonize the sales tax, income tax, and the federal excise tax laws with a view to facilitate the work of the functionally structured tax administration (structural benchmark). This harmonization will also include the elimination of the option for retailers with a turnover over Rs. 10 million to choose the presumptive tax regime. These measures will be taken prior to the Fund's Board consideration of the third review.

- On July 18, 2009, the FBR adopted its action plan on tax administration reform for the period July-December 2009. As part of this plan, we will reinforce our efforts to:

Strengthen and speed up tax audits. To that effect, we will audit 15 percent of the LTU taxpayers and 2 percent of existing medium-size business taxpayers by end-December 2009;

$>\quad$ Reduce the number of unregistered taxpayers, stop filers, and under-reporting. In this context, by end-September 2009, we expect to reduce sales tax and corporate tax nonfilers (registered taxpayers who do not file returns on time) to less than 1 percent in the case of existing LTU taxpayers, and to less than 7 percent for nonfilers that are medium-size business taxpayers.

$>\quad$ Introduce a 1 percent withholding mechanism on sales taxes administered by the FBR on purchases made by large taxpayers.

- $\quad$ To prepare for the introduction of a broad-based VAT in July 2010, we will ensure that the new expedited sales tax refund system is operational in all LTUs and RTOs by end-December 2009, and ready for full implementation by July 1, 2010, as part of the new VAT system.

- We plan to staff fully the Revenue Division of the Ministry of Finance and complete the shift of tax policy functions from the FBR to this division by end-December 2009.

18. Strengthening the social safety net remains a key program objective. The roll-out of the poverty scorecard system for the selection of beneficiary families under the Benazir Income Support Program (BISP) in 16 pilot districts may take somewhat longer than expected, given the difficulties in building administrative capacity. Nevertheless, we expect the roll-out of the scorecard to all 130 districts to be completed between December 2009 and June 2010. Using the original selection method of beneficiary families, 1.75 million families have been approved at end-June 2009, compared to an original target of 3 million. Hence, BISP disbursements in 2008/09 were lower than budgeted. However, delays in BISP disbursements in 2008/09 are not expected to impact disbursements in 2009/10 as beneficiary families identified during 2009/10 will receive benefits for the entire fiscal year, independent of the timing of approval of eligibility during 2009/10. 
19. We will continue to strengthen our expenditure controls. This will be achieved by implementing the medium-term budget framework at the federal level and strengthening, as well as extending the operations of the Project for Improving Financial Reporting and Auditing (PIFRA) to monthly federal and provincial fiscal reporting. A review of PIFRA to improve the coverage and quality of fiscal reporting will be undertaken and IMF technical assistance will be requested in this regard. These measures will help us to manage the disbursements of Tokyo pledges and to better control expenditures after this additional source of financing disappears. The transition to a Treasury Single Account (Federal Consolidated Fund) will be completed gradually. The government has already introduced a key element of a Treasury Single Account, requiring that all disbursements pass through a zero balance assignment account. We have also reduced the stock of outstanding balances with commercial banks. Following a World Bank technical assistance mission in July 2009, the remaining balances with commercial banks will be surveyed, reduced, and transferred to the Federal Consolidated Fund by end-June 2010, while ensuring the reduction does not affect banking sector liquidity negatively.

\section{We have reached understandings with World Bank and ADB staffs on a plan for} eliminating electricity tariff differential subsidies by August 2010. To that effect, average electricity tariffs will be increased in stages to ensure that they reach cost recovery in August 2010. The additional need for tariff differential subsidy of Rs. 55 billion in 2009/10 will be absorbed by the budget. Moreover, the National Electric Power Regulatory Authority (NEPRA) Act will be amended by July 31, 2009 (prior action) and implemented immediately to ensure: (i) monthly determination by NEPRA of the fuel/power purchase price adjustments in line with international fuel prices and automatic implementation upon determination (the first adjustment in August 2009 will be made relative to prices prevailing in July 2009); and (ii) quarterly determination of overall electricity tariffs by NEPRA and notification of the adjusted tariffs within 15 days by the government beginning in August 2010 (during 2009/10 NEPRA will perform the quarterly determinations, but tariffs will be notified according to the agreed tariff adjustment schedule). Any review requests of tariffs will be completed within 15 days (should this not be the case, the results of the review will be taken into account in the subsequent determination). Moreover, to address the issue of circular debt, the government has decided to assume debt (Rs. 277 billion, the equivalent of 1.9 percent of GDP) that was contracted by electricity companies over the last few years on account of insufficient tariff increases and unpaid subsidies. All debt from 2008/09 and earlier years, slated to be assumed by the budget, will be audited by an internationally-renowned auditing firm by end-September 2009.

\section{We recognize that the implementation of the 2009/10 budget will be challenging} and have prepared measures to ensure the achievement of the deficit target. External and domestic risks are significant and could result in lower revenue and/or higher expenditures. In addition, there is a need to accommodate tariff differential subsidies in the amount of Rs. 55 billion, higher-than-budgeted interest payments for electricity sector debt assumed by the budget (Rs. 10 billion), and lower-than-budgeted petroleum levy revenues 
(Rs. 10 billion). Accordingly, we have decided to reduce nonpriority development spending with a view to achieving the agreed deficit target. Should further expenditure cuts become necessary to compensate for lower-than-currently-projected revenues or higher-thancurrently-projected expenditures, social safety net spending and other priority spending for IDPs, infrastructure, and the social sectors will not be subject to cuts.

\section{To ensure an orderly budget execution, a portion of the Fund resources to be} disbursed during July 2009-April 2010 could be used to finance the budget. To avoid delays in priority outlays owing to the timing of disbursement of donor support pledged in Tokyo a part of Fund resources ( $\$ 1.4$ billion or 0.8 percent of GDP) to be disbursed during July 2009-April 2010 could be used to finance the budget. However, Fund resources would not be used to cover delays or shortfalls in disbursements from multilateral donors. If there are delays in disbursements of nonmultilateral foreign assistance in excess of the amounts covered by Fund resources, the budget deficit target will be adjusted as described in $\uparrow 25$ of the revised Technical Memorandum of Understanding (TMU).

23. We plan to review the limits on the issuance of guarantees, which has increased in recent years. We have also started to publish, on a monthly basis, on the website of the Ministry of Finance the total outstanding stock of government guarantees, in line with the Fiscal Responsibility and Debt Limitation Act.

\section{B. Monetary and Exchange Rate Policy}

\section{The SBP's primary monetary policy objective is to reduce inflation while} continuing to strengthen the international reserve position. The SBP's liquidity management will also seek to ensure the smooth functioning of the domestic market, with the government issuing T-bills and other market-based instruments to avoid SBP financing of the budget. While subsidiary to its primary objective, the SBP will also calibrate the monetary policy stance to balance concerns about the slowdown in economic activity with the need to lower inflation. As the fiscal position is still under pressure, the timing of external disbursements is highly uncertain, and there is a need to combat inflationary pressures stemming from higher oil and electricity prices and increased public sector wages, any further reduction in the SBP policy rate will await a significant and sustained decline in core inflation.

25. The SBP will further improve its monetary operations framework by introducing an explicit corridor for the overnight money market rate by mid-August, 2009, market conditions permitting. This will enhance market signaling, strengthen the effectiveness of liquidity management, and reduce volatility in short-term market interest rates. It is expected that this change in the operational framework will also improve the transmission of monetary policy signals and strengthen their role in supporting price stability. The introduction of the corridor should not constrain the SBP's ability to respond to external and fiscal shocks. However, it will likely require increasing the frequency of reviews of the SBP's policy rate 
and enhancing coordination between the SBP and the government to improve the projections of financial flows and enhance communication of such information to the SBP in order to strengthen its liquidity forecasting framework.

\section{The SBP will continue to pursue a flexible exchange rate policy to strengthen the} external position and competitiveness. In this context, interventions in the foreign exchange market will be largely aimed at achieving the program's net foreign asset targets. The SBP has already notified the market that it will cease to provide foreign exchange for imports of diesel and other refined products effective August 1, 2009. Market conditions permitting, the SBP will accelerate its plan to phase-out of the provision of foreign exchange for oil imports by end-December 2009 .

\section{The SBP issued a circular on June 12, 2009, which eliminates the exchange} restrictions related to the imposition of the cash margin requirements on letters of credit, including those introduced since the inception of the IMF program.

\section{The SBP will rationalize its refinancing schemes and eliminate the subsidies} resulting from below market interest rates. The interest rate for the Export Financing Scheme (EFS) and the Long-Term Financing Facility (LTFF) will be increased in steps to the level of the weighted average yield on six-month T-bills and yields of the same tenor for Pakistan Investment Bonds (PIBs), respectively. The process of increasing these interest rates will begin in September 2009; by end-September 2010 they will reach levels 2 percentage points below the weighted average yield on six-month T-bills and PIBs of the same tenor for the EFS and LTFF, respectively; and by end-September 2011 they will equal the weighted average yield on six-month T-bills for the EFS and yields of the same tenor for PIBs for the LTFF.

\section{Financial Sector and Safeguards Issues}

\section{The financial sector remains sound, but pressures are increasing owing to the} slowdown in economic growth. The financial sector continues to exhibit resilience, and we are committed to continue monitoring it closely. The credit exposure of banks to vulnerable sectors of economy, like textiles, poses the main risk for the financial sector. The SBP has responded with several measures to ease pressure on banks while maintaining high standards for a sound banking system. Gross nonperforming loans (NPLs) increased from 9.1 percent of total loans in December 2008 to 11.5 percent in March 2009, with net NPLs to net loans rising from 2.5 percent to 3.9 percent over the same period. The SBP is encouraging banks to resolve the issue of growing NPLs through early recognition of problem loans and engagement with their borrowers for rescheduling or restructuring, when warranted. The capital position of the banking system remains strong, with a risk-weighted capital adequacy ratio of around 12.9 percent. Given the recent increases in NPLs, the SBP has strengthened the coverage of credit risk during its onsite examinations, continues to monitor closely NPLs 
in all banks, and has asked those banks with high NPLs to adopt appropriate corrective measures.

30. Updated stress tests indicated that the banking system remains generally resilient. Against this positive background, stress tests revealed increased vulnerability to default from large exposures and an increase in maturity mismatches between bank assets and liabilities in the $0-3$ months and over one-year categories (liquidity risk), which in both cases have gone beyond the 10 percent limit. This is being addressed by weekly monitoring of the liquidity profile of individual banks. In the last couple of months, liquidity profile in terms of the assets to deposits ratio and the quantum of liquid assets has improved substantially. The analysis of operational risk also shows an increased incidence of fraud. SBP has encouraged banks to improve the quality of their fraud control systems, and most of the large and mid-size banks have either implemented or are in the process of implementing sophisticated core banking systems.

31. Amendments to the Banking Companies Ordinance have been prepared to enhance the effectiveness of SBP enforcement in the area of banking supervision. These amendments have been reviewed and approved by the SBP Board, have been submitted to the government, and will be presented to parliament by end-August, 2009. The new Problem Bank Management and Contingency Plan has been adopted by the SBP, and a Federal Government guaranteed scheme for providing liquidity to small banks is in place.

32. The SBP recently reduced the quantum of gradual increments in minimum capital requirements for banks. However, enhancements in capital adequacy requirements (CARs) were maintained. The minimum amount of capital to be required for banking institutions at end-2013 was reduced from Rs. 23 billion to Rs. 10 billion (to be achieved progressively, starting with Rs. 6 billion by end-2009), but the minimum CAR has been increased to 10 percent, with this level to be achieved by $31^{\text {st }}$ December 2009 . The decision to reduce the minimum amount of capital was made to avoid undue concentration in the sector and foster increased competition.

\section{A new draft SBP law to enhance the SBP's operational independence has been} reviewed by the SBP Board. The draft SBP law includes the creation of an independent Monetary Policy Committee composed of members with strong professional credentials. The draft SBP law has recently been submitted to the inter-agency committee dealing with this issue. The government will present the respective legislative proposals to parliament as amendments to the SBP law by end-September 2009.

34. A new bankruptcy law will shortly be presented to the government. The new law will help with the rehabilitation of weak but viable corporate entities and the expeditious liquidation of other insolvent companies to address the issue of rising nonperforming bank loans. The new law will be submitted to parliament by end-August 2009. 


\section{The National Investment Trust's State Enterprise Fund (NITSEF) has performed} well. As of June 30, the NITSEF had invested in the stock market Rs. 10.3 billion of its resources, which total to Rs. 20 billion. The NITSEF has benefited both from a careful timing of purchases and the performance of the Karachi Stock Exchange. The NITSEF has a website where it states its investment strategy and discloses its financial statements, as well as the quantity and average acquisition price of security purchases at the end of each quarter.

36. In line with Fund policies related to the augmentation of resources, an updated safeguards assessment of the SBP will be conducted. This assessment will focus on developments since the recent assessment; in particular, actions taken in respect of the recommendations made to enhance the SBP's independence, further improve internal controls, and establish a Board Committee to centralize risk management activities.

\section{Program Risks AND Monitoring}

37. The program remains subject to considerable risks. The economic situation in Pakistan's trading partners could deteriorate further and result in lower demand for exports, workers' remittances, and private capital flows. In addition, delays in mobilizing disbursements of donor support would limit the scope for a countercyclical fiscal policy and add to the pressures on the budget. Also, adverse security developments could act as a drag on confidence in the economy and the recovery of domestic and external demand could take longer than anticipated, undermining the revenue targets. Moreover, as economic activity weakens, the risks to the financial sector may increase.

38. The program will remain subject to quarterly program reviews and quarterly performance criteria for 2009/10, as specified in Table 1. The attached TMU updates definitions for the adjustors related to external financing and, effective July 1, 2009 replaces the TMU dated November 20, 2008.

39. The following measure will be a prior action for Board consideration of the staff report for the second review: A presidential ordinance will be issued to amend the NEPRA Act to provide for (i) monthly determination by NEPRA of the fuel/power purchase price adjustments in line with international fuel prices and automatic implementation upon determination; (ii) quarterly determination of overall electricity tariffs by NEPRA to reflect all other cost components; and (iii) automatic notification of new tariffs within 15 days from NEPRA determination starting in August 2010.

40. We propose to convert the two missed end-June structural performance criteria into structural benchmarks. In line with the Fund's new review-based conditionality, we propose to convert the structural performance criterion on amendments to the banking legislation into a structural benchmark for end-August 2009, and the structural performance criterion on legislative amendments to harmonize administration of the income tax and GST laws into a structural benchmark for end-September 2009. 


\section{Program Financing}

41. Additional financing, including through higher access under the $S B A$, is needed to further strengthen the international reserves position in order to reduce the risks to the program. Further, the augmentation of access under the SBA will help manage the risks stemming from the global financial crisis. As noted, while the program remains financed for 2008/09-2009/10, the pledges made in Tokyo provide some room for countercyclical fiscal policy, including through social safety expenditures, development spending, and outlays to enhance security. It is crucial that the assistance pledged in Tokyo be disbursed promptly to address these needs. 
Table 1. Pakistan: Quantitative Targets, December 2008-June 2010

\begin{tabular}{|c|c|c|c|c|c|c|c|}
\hline & $\begin{array}{l}\text { Outst. Stock } \\
\text { end-Sept. } \\
2008\end{array}$ & $\begin{array}{l}\text { end-Mar. } \\
2009\end{array}$ & $\begin{array}{l}\text { end-Jun. } \\
200921\end{array}$ & $\begin{array}{c}\text { Prog. 3/ } \\
\text { end-Sept. } \\
2009\end{array}$ & $\begin{array}{c}\text { Prog. 3/ } \\
\text { end-Dec. } \\
2009\end{array}$ & $\begin{array}{c}\text { Indicative } \\
\text { end-Mar. } \\
2010\end{array}$ & $\begin{array}{c}\text { Indicative } \\
\text { end-Jun. } \\
2010\end{array}$ \\
\hline Floor on net foreign assets of the SBP* (stock, in millions of U.S. dollars) & 3,953 & 671 & 2,782 & 3,200 & 3,250 & 3,100 & 3,250 \\
\hline Adjusted target & $\ldots$ & 446 & N/A & & & & \\
\hline Actual & $\ldots$ & 3,132 & 3,982 & & & & \\
\hline Ceiling on net domestic assets of the SBP* (stock, in billions of Pakistani rupees) & 1,250 & 1,412 & 1,314 & 1,300 & 1,340 & 1,365 & 1,410 \\
\hline Adjusted target & $\ldots$ & 1,416 & N/A & & & & \\
\hline Actual & $\ldots$ & 1,201 & 1,183 & & & & \\
\hline Ceiling on net government borrowing from SBP* (stock, in billions of Pakistani rupees) & 1,227 & 1,274 & 1,181 & 1,130 & 1,130 & 1,130 & 1,130 \\
\hline Adjusted target & $\ldots$ & 1,274 & N/A & & & & \\
\hline Actual & $\ldots$ & 1,071 & 1,130 & & & & \\
\hline Ceiling on overall budget deficit* (cumulative flow, in billions of Pakistani rupees) & 142 & 405 & 562 & 194 & 384 & 573 & 721 \\
\hline Adjusted target & $\ldots$ & 405 & N/A & & & & \\
\hline Actual & $\ldots$ & 405 & 683 & & & & \\
\hline \multicolumn{8}{|l|}{ Ceiling on outstanding stock of short-term public and publicly guaranteed external debt* (in millions } \\
\hline of U.S. dollars) & 515 & 1,500 & 1,500 & 1,500 & 1,500 & 1,500 & 1,500 \\
\hline Actual & $\ldots$ & 728 & 652 & & & & \\
\hline \multicolumn{7}{|l|}{$\begin{array}{l}\text { Cumulative ceiling on contracting of nonconcessional medium- and long-term public and publicly } \\
\text { guaranteed external debt* (in millions of U S dollars) } 1 /\end{array}$} & 9,500 \\
\hline Actual & $\ldots$ & 1,299 & 1,649 & & & & \\
\hline \multicolumn{8}{|l|}{ Accumulation of external payments arrears (continuous performance criterion during the program } \\
\hline Actual & $\ldots$ & 0 & 0 & & & & \\
\hline Continuous ceiling on SBP's foreign currency swaps and forward sales* (in millions of U.S. dollars) & 1,900 & 2,750 & 2,750 & 2,500 & 2,500 & 2,500 & 2,500 \\
\hline Actual & $\ldots$ & 1,835 & 1,690 & & & & \\
\hline \multicolumn{8}{|l|}{ Memorandum items: } \\
\hline Net external program financing (cumulative, in millions of U.S. dollars) & -166 & 296 & 616 & 95 & -517 & $-1,067$ & 274 \\
\hline Of which: Use of IMF resources by the budget (cumulative, in millions of U.S. dollars) & $\ldots$ & $\ldots$ & $\ldots$ & 715 & 1,072 & 1,430 & 0 \\
\hline Tokyo pledges, excl. multilateral disbursements (cumulative, in millions of U.S. dollars) & $\ldots$ & $\ldots$ & $\ldots$ & 342 & 1,177 & 1,962 & 2,220 \\
\hline Tokyo-related disbursements from multilateral sources & $\ldots$ & $\ldots$ & $\ldots$ & 0 & 0 & 100 & 270 \\
\hline External project grants (in millions of U.S. dollars) & 24 & 111 & 154 & 320 & 650 & 908 & 991 \\
\hline Budgetary grants for IDPs & $\ldots$ & $\ldots$ & $\ldots$ & 110 & 183 & 202 & 220 \\
\hline \multicolumn{8}{|l|}{ Foreign Exchange cash reserve requirement (CRR, incl. special CRR) deposits in SBP (in millions } \\
\hline of U.S. dollars) & 832 & 745 & 728 & 750 & 770 & 790 & 800 \\
\hline \multicolumn{8}{|l|}{ Weekly cash reserve requirement ratios (in percentage points) } \\
\hline Rupee deposits (less than one year maturity) & 9 & 5 & 5 & 5 & 5 & 5 & 5 \\
\hline Rupee deposits (more than one year maturity) & 0 & 0 & 0 & 0 & 0 & 0 & 0 \\
\hline Foreign currency deposits CRR & 5 & 5 & 5 & 5 & 5 & 5 & 5 \\
\hline Foreign currency deposits special CRR & 15 & 15 & 15 & 15 & 15 & 15 & 15 \\
\hline Increase in SDR allocation (stock in millions of U.S. dollars) & $\ldots$ & $\ldots$ & $\ldots$ & 0 & 1,240 & 1,240 & 1,240 \\
\hline
\end{tabular}

Notes:

* denotes performance citeria.

1/Excludes IMF

2/ All end-June 2009 figures shown as "Actual" are reported on a preliminary basis.

$3 /$ In addition to the quantitative performance criteria for end-September and end-December, the relevant purchases will also be subject to the completion of reviews: third review to be completed no earlier than November 29, 2009, and fourth review to be completed no earlier than February 27, 2010. 
Table 2. Pakistan: Structural Conditionality, December 2008-December 2009

\begin{tabular}{|c|c|c|}
\hline & Target date & Status \\
\hline \multicolumn{3}{|l|}{ Prior Action } \\
\hline $\begin{array}{l}\text { Amendment of the NEPRA Act, by a presidential decree, to provide for: (i) monthly } \\
\text { determination by NEPRA of the fuel/power purchase adjustments in line with } \\
\text { international fuel prices and automatic implementation upon determination; (ii) quarterly } \\
\text { determination of overall electricity tariffs by NEPRA to reflect all cost components; and } \\
\text { (iii) automatic notification of new tariffs within } 15 \text { days from NEPRA determination } \\
\text { starting in August } 2010 \text {. }\end{array}$ & end-July 2009 & \\
\hline \multicolumn{3}{|l|}{ Structural Benchmarks } \\
\hline A contingency plan for handling problem private banks will be finalized. & end-December 2008 & Met \\
\hline $\begin{array}{l}\text { A full description of required reforms in the area of tax administration, including an } \\
\text { action plan for harmonizing the GST and income tax administration will be finalized. }\end{array}$ & end-December 2008 & Met \\
\hline $\begin{array}{l}\text { In close collaboration with the World Bank, the government will finalize the schedule } \\
\text { for further electricity tariff adjustments during } 2008 / 09 \text {, with a view to eliminating } \\
\text { tariff differential subsidies by end-June } 2009 \text {. }\end{array}$ & end-December 2008 & Met $1 /$ \\
\hline The SBP's provision of foreign exchange for furnace oil will be eliminated. & February 1, 2009 & Met \\
\hline $\begin{array}{l}\text { In close collaboration with the World Bank, the government will develop a strategy } \\
\text { and a time-bound action plan for the adoption of specific measures to strengthen } \\
\text { the social safety net and improve targeting to the poor. }\end{array}$ & end-March, 2009 & Met \\
\hline The government will prepare a plan for eliminating the inter-corporate circular debt. & end-March, 2009 & Met with a delay \\
\hline The transition to a single treasury account will be completed. & end-June 2009 & Underway $2 /$ \\
\hline $\begin{array}{l}\text { Amendments to the banking legislation will be submitted to Parliament to enhance } \\
\text { the effectiveness of SBP enforcement powers in the area of banking supervision. }\end{array}$ & end-August 2009 3/ & \\
\hline $\begin{array}{l}\text { 1. Government approval of regulations to (i) form new occupational groups within the FBR; } \\
\text { and (ii) revise the structures of Regional Taxpayer Offices and Large Taxpayer Units. } \\
\text { 2. Amendment of all relevant laws and rules. }\end{array}$ & September 15, 2009 & \\
\hline $\begin{array}{l}\text { The government will submit draft legislative amendments to parliament to harmonize } \\
\text { the income tax and GST laws, including for tax administration purposes. }\end{array}$ & end-September 2009 4/ & \\
\hline Submission of the VAT law to Parliament & end-December 2009 & \\
\hline
\end{tabular}

1/ The original plan was superseded by a plan that was agreed upon on July 15, 2009.

$2 /$ In October 2008, the authorities introduced a system preventing accumulation of unspent balances in accounts outside the Federal Consolidated Fund and made the use of assignment accounts by ministries under the Account No.1 fully operational for budget expenditures. However, a significant number of accounts at commercial banks with unspent balances remains.

3/ Formerly a structural performance criterion with target date at end-June 2009 (missed). Proposed to be converted into a structural benchmark.

4/ Formerly a structural performance criterion with target date at end-June 2009 (missed). Proposed to be converted into a structural benchmark. The original structural performance criterion also targeted a reduction in exemptions. This component is not included in the proposed benchmark. 


\section{Attachment III. Pakistan: Technical Memorandum of Understanding (TMU) on The Program Supported Under The StANd-By ARrangement}

July 29, 2009

1. With effect from July 1, 2009, this Technical Memorandum of Understanding (TMU) describes the monitoring arrangements under the SBA-supported program. Throughout, unless otherwise stated, "government" is meant to comprise the federal and provincial governments.

\section{Definitions of Monitoring VARiables}

\section{Valuation of foreign exchange denominated assets, liabilities, and foreign exchange flows}

2. For the purposes of program monitoring under the program, all assets and liabilities as well as debt contracted, denominated in SDRs or in currencies other than the U.S. dollar, will be converted into U.S. dollars at the exchange rates prevailing at test dates, as posted by the State Bank of Pakistan (SBP) on its web site. Net external budget financing and external cash grants will be converted into Pakistani rupees at the exchange rates prevailing at the day of the transaction, as posted by the SBP on its web site, unless otherwise indicated.

3. Reserve money (RM) is defined as the sum of: currency outside scheduled banks (deposit money banks); scheduled banks' domestic cash in vaults; scheduled banks' required and excess rupee; foreign exchange deposits with the SBP; and deposits of the rest of the economy with the SBP, excluding those held by the federal and provincial governments and the SBP staff retirement accounts.

4. Net foreign assets (NFA) of the SBP are defined as the difference between its foreign assets and foreign liabilities. Foreign assets of the SBP consist of gold, foreign exchange, balances held outside Pakistan, foreign securities, foreign bills purchased and discounted, the reserve position with the IMF, and SDR holdings. The definition of foreign assets of the SBP will be consistent with the IMF Data Template on International Reserves and Foreign Currency Liquidity. Gold will be valued at $\$ 20.27$ per troy ounce per fine troy ounce. Foreign liabilities of the SBP include outstanding IMF credits (excluding net IMF financing to the budget as defined in paragraph 10 below), deposits with the SBP of foreign governments, foreign central banks, foreign deposit money banks, international organizations, and foreign nonbank financial institutions (NBFI). 
5. Net domestic assets (NDA) of the SBP are defined as the difference between the RM and the NFA of the SBP.

6. Net borrowing from the banking system by the government is defined as the difference between the banking system's claims, on a cash basis, on the federal, provincial, and local governments and the deposits of the federal, provincial, and local governments with the banking system, including district government funds balances. For the purposes of this memorandum, claims on government exclude: credit for commodity operations; government deposits exclude outstanding balances in the Zakat Fund; and balances in the various privatization accounts kept by the government in the banking system. The stock of bonds which were issued to banks in substitution of outstanding nonperforming loans to certain public entities, and which are being fully serviced by the government, are included in banking system claims on government. Table 1 summarizes the calculations of net borrowing from the banking system by the government.

7. Net borrowing from the SBP by the government is defined as SBP claims on the government minus government deposits with the SBP. SBP claims on the government include government securities, treasury bills, ways and means advances, treasury currency, and debtor balances. SBP claims on the government exclude accrued profits on government securities. Government deposits with the SBP exclude the Zakat Fund and Privatization accounts (Table 1).

8. The definition of the overall budget deficit (excluding grants) under the program will be the consolidated budget deficit, excluding grants, and including the operations of district governments financed from local funds. It will be measured by the sum of (a) total net financing to the federal, provincial, and local governments; and (b) total external grants to the federal and provincial governments. The former is defined as the sum of (i) net external budget financing (see 911 ); (ii) net borrowing from the banking system (as defined above); and (iii) net domestic nonbank financing (see $\$ 12$ ). The total external grants are defined as the sum of project grants, cash external grants for budgetary support, capital grants reflecting the principal amounts of external debt cancellation or swaps, and other grants.

9. Net external program financing is defined to include external privatization receipts; budget support grants; budget support loans from multilateral (other than the IMF, but including World Bank and Asian Development Bank (ADB) budget support and structural adjustment loans), official bilateral budget support loans, and private sector sources (e.g., bonds); rescheduled government debt service and change in stock of external debt service arrears net of government debt amortization due on foreign loans, the latter including any accelerated amortization including related to debt swaps or debt cancellation recorded as capital grants. It also includes foreign loans onlent to financial institutions and companies 
(public or private) and emergency relief lending. Program financing excludes all external financing counted as reserve liabilities of the SBP (defined above). Amounts projected for net external program financing and external grants are provided in Table 2.

10. Net IMF financing to the budget is defined as the difference between Fund disbursements credited to the budget and repayments from the budget through the Ministry of Finance's dedicated account for Fund financing at the SBP.

11. Net external budget financing is defined as net external program financing minus privatization receipts, minus budget support grants, plus all other external loans for the financing of public projects or other federal or provincial budget expenditures, plus transfers of external privatization receipts from the privatization account to the budget, plus net IMF financing to the budget. Amounts projected for net external budget financing are provided in Table 2.

12. Net domestic nonbank financing of the budget is defined as follows: domestic privatization receipts transferred from the privatization accounts to the budget, plus the change during each reporting period, in the stock of (i) permanent debt, which consists of nonbank holdings of prize bonds, all federal bonds, and securities; plus (ii) floating debt held by nonbanks; plus (iii) unfunded debt, which consists of National Savings Scheme (NSS) debt, Postal Life Insurance, and the General Provident Fund (GPF); plus (iv) net deposits and reserves received by the government (public accounts deposits); plus (v) any other government borrowing from domestic nonbank sources net of repayments; minus (vi) government deposits with NBFIs. Nonbank holdings of permanent and floating debt is defined as total debt outstanding, as reported by the SBP, minus holdings of banks as per the monetary survey. Total T-bill and other relevant government debt are valued at discount value and exclude accrued interest.

\section{External debt}

13. The performance criterion on contracting or guaranteeing of medium-term and long-term nonconcessional external debt by the government or the SBP applies not only to debt as defined in point No. 9 of the Guidelines on Performance Criteria with Respect to Foreign Debt (adopted by the IMF Executive Board on August 24, 2000), but also to 
commitments contracted or guaranteed for which value has not been received. ${ }^{1}$ Excluded from this performance criterion are (i) foreign currency deposit liabilities of the SBP; and (ii) the outstanding stock of debt of Foreign Exchange Bearer Certificates (FEBCs), Deposit Bearer Certificates (DBCs), and Foreign Currency Bearer Certificates (FCBCs). The performance criterion setting a limit on the outstanding stock of short-term external debt refers to debt (as defined in Footnote 1) with original maturity of up to and including one year. Medium- and long-term external debt comprises debt with initial maturity of over one year.

14. Nonconcessional borrowing is defined as borrowing with a grant element of less than 35 percent. $^{2}$ The discount rates used to calculate the grant element will be the six-month and ten-year Commercial Interest Reference Rates (CIRRs) averages, as computed by the Strategy and Policy Review Department of the IMF. Six-month CIRRs are updated midFebruary and mid-August (covering the six-month period preceding the date of update) and the ten-year CIRRs averages are updated mid-December (covering a period of 10 years preceding the date of the update). Six-month CIRRs averages are to be used for loans whose maturity is less than 15 years, while ten-year CIRRs averages are to be used for loans whose maturity is equal or more than 15 years.

15. External payment arrears are defined as unpaid debt service by the government beyond the due date under the contract and any grace period.

\footnotetext{
${ }^{1}$ The definition of debt set forth in No. 9 of the guidelines reads as follows: "(a) For the purpose of this guideline, the term "debt" will be understood to mean a current, that is, not contingent, liability, created under a contractual arrangement through the provision of value in the form of assets (including currency) or services, and which requires the obligor to make one or more payments in the form of assets (including currency) or services, at some future point(s) in time; these payments will discharge the principal and/or interest liabilities incurred under the contract. Debts can take a number of forms, the primary ones being as follows: (i) loans, that is, advances of money to obligor by the lender made on the basis of an undertaking that the obligor will repay the funds in the future (including deposits, bonds, debentures, commercial loans, and buyers' credits) and temporary exchanges of assets that are equivalent to fully collateralized loans under which the obligor is required to repay the funds, and usually pay interest, by repurchasing the collateral from the buyer in the future (such as repurchase agreements and official swap arrangements); (ii) suppliers' credits, that is, contracts where the supplier permits the obligor to defer payments until some time after the date on which the goods are delivered or services are provided; and (iii) leases, that is, arrangements under which property is provided which the lessee has the right to use for one or more specified period(s) of time that are usually shorter than the total expected service life of the property, while the lessor retains the title to the property. For the purpose of the guideline, the debt is the present value (at the inception of the lease) of all lease payments expected to be made during the period of the agreement excluding those payments that cover the operation, repair or maintenance of the property. (b) Under the definition of debt set out in point 9 (a) above, arrears, penalties, and judicially awarded damages arising from the failure to make payment under a contractual obligation that constitutes debt are debt. Failure to make payment on an obligation that is not considered debt under this definition (for example, payment on delivery) will not give rise to debt."

${ }^{2}$ Following the methodology set out in "Limits on External Debt or Borrowing in Fund ArrangementsProposed Change in Implementation of the Revised Guidelines," April 8, 1996.
} 
16. Tokyo-related disbursements refers to disbursements of donor support (loans and grants) pledged at the donor conference held in Tokyo on April 17, 2009.

\section{Adjustors}

\section{Adjustors related to net external program financing}

17. For paragraphs $18-23$, the net external program financing excludes any budget grants related to Internally Displaced Persons (IDPs), program portion of Tokyo-related disbursements, and Fund financing to the budget. They are projected as follows:

July 2009-September 2009: \$95 million

July 2009-December 2009: -\$517 million

July 2009-March 2010: -\$1,067 million

July 2009-June 2010: \$274 million

18. If the actual cumulative net external program financing in U.S. dollar terms is higher than its projected value by more than $\$ 500$ million, the excess net external program financing in U.S. dollar terms is defined as follows: actual external program financing in U.S. dollar terms minus projected net external program financing in U.S. dollar terms minus $\$ 500$ million. The excess net external program financing in U.S. dollar terms multiplied by a fixed accounting exchange rate of Rs. 83 per $\$ 1$ represents the excess net external program financing in rupee terms.

19. The ceiling on the consolidated overall budget deficit (excluding grants) will be adjusted upward for the cumulative excess in net external program financing in rupee terms for up to Rs. 73.5 billion at end-September 2009, Rs. 73.5 billion at end-December 2009, Rs. 73.5 billion at end-March 2010, and Rs. 73.5 billion at end-June 2010.

20. The cumulative excess net external program financing in U.S. dollar terms may exceed the cumulative maximum limits specified in paragraph 17 for end-September 2009, end-December 2009, end-March 2010, and end-June 2010, when converted into U.S. dollar terms at a fixed accounting exchange rate of Rs. 83 per $\$ 1$. In such a case, the cumulative excesses in net external program financing in U.S. dollar terms minus the maximum cumulative amounts specified for end-September 2009, end-December 2009, end-March 2010, and end-June 2010 converted into U.S. dollar terms at a fixed accounting exchange rate of Rs. 83 per $\$ 1$ are defined as "surplus net external program financing in U.S. dollar terms." The latter amount multiplied by a fixed accounting exchange rate of Rs. 83 per $\$ 1$ constitutes "surplus net external program financing in rupee terms."

21. The floors on the NFA of the SBP will be adjusted upward by the cumulative surplus net external program financing in U.S. dollar terms as defined above. 
22. The ceilings on the NDA of the SBP and net borrowing from the SBP by the government will be adjusted downward by the cumulative surplus net external program financing in rupee terms as defined above.

23. If the actual cumulative net external program financing in U.S. dollar terms is lower than its projected value, the shortfall in net external program financing in U.S. dollar terms is defined as the difference between its projected and actual values in U.S. dollar terms. In such a case:

a. The floor on the NFA of the SBP is adjusted downward by the cumulative shortfall in net external program financing in U.S. dollar terms.

b. The ceiling on the NDA of the SBP is adjusted upward by the cumulative shortfall in net external program financing in U.S. dollar terms converted into rupees at a fixed accounting exchange rate of Rs. 83 per $\$ 1$.

c. The ceiling on net borrowing from the SBP by the government is not subject to adjustment.

\section{Adjustors related to Tokyo related disbursements (excluding multilateral sources)}

24. Tokyo related disbursements (excluding multilateral sources) are projected as follows:

July 2009-September 2009: \$342 million

July 2009-December 2009: \$1,177 million

July 2009-March 2010: \$1,962 million

July 2009-June 2010: \$2,220 million

25. If the actual cumulative gross Tokyo related disbursements (excluding multilateral sources) is lower than the projected amount by more than $\$ 1.421$ billion, the difference between its projected and actual values in dollar terms is defined as the short fall in Tokyo related disbursements (excluding multilateral sources). The shortfall in Tokyo related disbursements (excluding multilateral sources) in U.S. dollar terms multiplied by a fixed accounting exchange rate of Rs. 83 per $\$ 1$ represents the shortfall in Tokyo related disbursements (excluding multilateral sources) in rupee terms. In such a case:

a. The ceiling on the consolidated overall budget deficit (excluding grants) is adjusted downward up to Rs. 29.49 billion at end-September 2009, Rs. 58.97 billion at end-December 2009, Rs. 58.97 billion at end-March 2010, and Rs. 58.97 billion at end-June 2010 (equivalent to $\$ 0.71$ billion valued at Rs. 83 per $\$ 1$ ) for shortfall in 
Tokyo related disbursements (excluding multilateral sources) in rupee terms in excess of Rs. 117.94 billion.

b. The floor on the NFA of the SBP is adjusted downward by the amount equivalent to the cumulative shortfall in Tokyo related disbursements (excluding multilateral sources) in U.S. dollar terms.

c. The ceiling on the NDA of the SBP is adjusted upward by the amount equivalent to the cumulative shortfall in Tokyo related disbursements (excluding multilateral sources).

\section{Adjustors related to Tokyo related disbursements from multilateral sources}

26. Tokyo related disbursements from multilateral sources are projected as follows: July 2009-September 2009: \$0 million July 2009-December 2009: \$0 million July 2009-March 2010: \$100 million July 2009-June 2010: \$270 million

27. If the actual cumulative gross Tokyo related disbursements from multilateral sources is lower than the projected amount, the difference between its projected and actual values in dollar terms is defined as the short fall in Tokyo related disbursements from multilateral sources. The shortfall in related disbursements Tokyo related disbursements from multilateral sources in U.S. dollar terms multiplied by a fixed accounting exchange rate of Rs. 83 per $\$ 1$ represents the shortfall in Tokyo related disbursements from multilateral sources in rupee terms. In such a case:

a. The ceiling on the consolidated overall budget deficit (excluding grants) is adjusted downward by the shortfall in Tokyo related disbursements from multilateral sources in rupee terms.

b. The floor on the NFA of the SBP is adjusted downward by the amount equivalent to the cumulative shortfall in Tokyo related disbursements from multilateral sources.

c. The ceiling on the NDA of the SBP is adjusted upward by the amount equivalent to the cumulative shortfall in Tokyo related disbursements from multilateral sources.

d. The ceiling on net borrowing from the SBP by the government will not be adjusted. 


\section{Adjustor related to net IMF financing to the budget}

28. Net IMF financing to the budget is projected as follows:

July 2009-September 2009: \$715 million

July 2009-December 2009: \$1,072 million

July 2009-March 2010: \$1,430 million

July 2009-June 2010: \$0 million

29. If actual net Fund financing to the budget in U.S. dollar terms is different from the projected amount, the difference in net Fund financing to the budget in U.S. dollar terms is defined as the difference in actual net Fund financing converted into U.S. dollar by the actual SDR/U.S. dollar exchange rates and the projected amount. In such a case,

a. The floor on the NFA of the SBP will be adjusted by the difference in Fund financing to the budget in U.S. dollar terms (upward if the difference is positive, downward if the difference is negative).

b. The ceiling on the NDA of the SBP will be adjusted by subtracting the difference in Fund financing to the budget in U.S. dollar terms converted into rupees at a fixed accounting exchange rate of Rs. 83 per $\$ 1$ (downward if the difference is positive, upward if the difference is negative).

\section{Adjustor related to SDR allocations}

30. If actual SDR allocation in U.S. dollar terms is different than projected amount (Table 2), the difference in SDR allocations in U.S. dollar terms is defined as the difference in actual disbursements converted into U.S. dollar by the actual SDR/dollar exchange rates and the projected disbursement in U.S. dollar terms. In such a case,

a. The floor on the NFA of the SBP will be adjusted by the difference in SDR allocations in U.S. dollar terms (upward if the difference is positive, downward if the difference is negative).

b. The ceiling on the NDA of the SBP will be adjusted by subtracting the difference in SDR allocations in U.S. dollar terms converted into rupees at a fixed accounting exchange rate of Rs. 83 per $\$ 1$ (downward if the difference is positive, upward if the difference is negative). 


\section{Adjustor related to project grants and budget grants related to IDP}

31. If the amount of project grants or budget grants related to IDP is higher or lower than assumed under the program (see Table 2), the ceiling on the consolidated overall budget deficit (excluding grants) will be adjusted upward or downward for the cumulative excess or shortfall in project grants or in budget grants related to IDPs respectively. Where applicable, this adjustor will not be applied in addition to any adjustment to the consolidated overall budget deficit (excluding grants) that is made under paragraphs 19 and 23.

\section{Adjustor related to changes in regulations on required reserves}

32. The ceilings on the NDA of the SBP will also be adjusted downward/upward by the amount of (i) banks' Pakistani rupee reserves freed/seized by any reduction/increase of the daily CRR relative to the baseline assumption; and (ii) any reduction/increase in the reservable deposit base that is related to definitional changes, as per the following formula: $\Delta \mathrm{NDA}=\Delta \mathrm{rB}_{0}+\mathrm{r}_{0} \Delta \mathrm{B}+\Delta \mathrm{r} \Delta \mathrm{B}$, where $\mathrm{r}_{0}$ denotes the reserve requirement ratio prior to any change; $\mathrm{B}_{0}$ denotes the level of the reservable deposits in the initial definition; $\Delta \mathrm{r}$ is the change in the reserve requirement ratio; and $\Delta \mathrm{B}$ denotes the change in the reservable deposits as a result of definitional changes. In case of significant liquidity and other financial sector pressures, the SBP will engage in consultations with the IMF staff in order to reach understanding on appropriate monetary policy response.

\section{Adjustor related to the SBP's net position under foreign exchange forwards and swaps}

33. An adjustor to the NFA target of the SBP will be implemented to reflect changes in the SBP's net position under foreign exchange forwards and swaps. Specifically, the NFA target of the SBP will be adjusted upward/downward by the amount of the increase/decrease in the net SBP's position under foreign exchange forwards and swaps. The maximum SBP's net exposure under foreign exchange forwards and swaps is capped at $\$ 2.75$ billion as of end-September 2009, end-December 2009, end-March 2010, and end-June 2010. The SBP's net exposure under foreign exchange forwards and swaps was \$1.69 billion at end-June 2009.

\section{Adjustor related to foreign currency deposits of resident banks with the SBP}

34. An adjustor to the NFA target of the SBP will be implemented to reflect changes in foreign currency deposits of resident banks. Specifically, the NFA target of the SBP will be adjusted upward/downward by the amount of increase/decrease in foreign currency deposits of resident banks with the SBP. The stock of foreign currency deposits with resident banks was $\$ 832$ million at end-June 2009. 


\section{Program Reporting ReQuirements}

35. The following information, including any revisions to historical data, will be provided to the Middle East and Central Asia Department of the IMF through the office of the Resident Representative of the IMF in Pakistan, within the timeframe indicated:

- Monthly provisional statements on federal tax and nontax revenue, within one month.

- Deposits into and withdrawals from the privatization accounts for each quarter, within one month. Withdrawals will be reported with the following breakdown (i) those which constitute budgetary use of privatization proceeds; (ii) those which constitute costs of privatization; and (iii) other (with explanation of the purpose of other withdrawals), as well as with the breakdown between domestic and external privatization receipts.

- Quarterly statements on budgetary capital receipts and disbursements, including repayments of bonds, recovery of loans from provinces and "others," within two months.

- Monthly (unreconciled) provisional data on federal expenditure and net lending (with separate data on disbursements and repayments), within one month.

- $\quad$ Quarterly statement on consolidated budgetary expenditure, with federal data approved by the Accountant General Pakistan Revenue (AGPR), within two months.

- Quarterly numbers on expenditure on social programs.

- Quarterly data on the stock of domestic government debt, broken down by instrument, within one month (Table 3).

- $\quad$ Quarterly data on WAPDA receivables within one month.

- $\quad$ Monthly data on Outstanding Audited Price Differential Claims.

- $\quad$ Monthly data on external budget financing, including (i) loan-by-loan program disbursements in U.S. dollar terms and rupee terms converted at exchange rates prevailing at the time of each transaction; (ii) cumulative amortization in U.S. dollar terms and rupee terms converted at exchange rates prevailing at the time of each transaction; and (iii) cumulative project loan disbursements in U.S. dollar terms and in rupees converted at exchange rates prevailing at the time of each transaction. Tokyo and IDP related disbursement should be indicated separately.

- Monthly data on Banks' Budgetary Support (Table 1) within one month. 
- The following monthly monetary data on a last-Saturday basis within two weeks:

(i) monetary survey;

(ii) accounts of the SBP;

(iii) consolidated accounts of the scheduled banks;

(iv) banks' lending to the government;

(v) detailed table on net foreign assets (both for the SBP and scheduled banks); and

(vi) detailed table of scheduled banks' reserves with the SBP.

- $\quad$ The same tables as in the preceding item, but on an end-month and end-quarter basis (last business day), both at current and program exchange rates, within one month.

- $\quad$ The SBP Table on outstanding stock of foreign currency deposits, amended to include the classification of new FCA according to the residency of the holder.

- Daily data on exchange rates (interbank, retail market, and Telegraphic Transfers for SBP purchases in the retail market), SBP's sales and purchases in the foreign exchange markets, swaps and forward outright sales, within two business days.

- $\quad$ Monthly data on the outstanding stock of the SBP's forward foreign currency operations, including swaps and outright forward sales and purchases, within two weeks. The terms of any new transactions (including rollover/renewal of existing ones) will also be provided.

- Monthly data on the SBP's foreign exchange reserves, with details on the currencies, instruments, and institutions in which the reserves are held, within one month.

- Monthly data on SBP direct or bridge loans to nationalized banks in the context of the restructuring and privatization operation, within four weeks.

- Monthly data on any other quasi-fiscal operations undertaken by the SBP, on behalf of the government.

- Monthly data on SBP holding of discounted export finance credit under the export finance scheme, within one month.

- Monthly data on outstanding credit to agriculture under the Agriculture Mandatory Credit Targets, within one month.

- The following data on external debt, within one month:

(i) Quarterly stock of public- and publicly-guaranteed external debt (including deferred payments arrangements), by maturity (initial maturities of up to and including one year, and over one year), by creditor and by debtor (central government and publicly guaranteed); 
(ii) Quarterly contracting or guaranteeing of nonconcessional medium- and long-term government debt; and

(iii) Information on any rescheduling on public- and publicly-guaranteed debt reached with creditors.

- Quarterly data on external payments arrears on public and publicly guaranteed debt with details as in (i) of the preceding item within one month.

- $\quad$ Copies of new or revised ordinances/circulars regarding changes in tax policy, tax administration, foreign exchange market regulations, and banking regulations no later than three days after official issuance, or notification that ordinances have been posted on the Federal Board or Revenue (FBR) and SBP websites.

- $\quad$ Copies of official notification of changes in gas and electricity tariffs and any surcharges (automatic or structural) and in ex-refinery petroleum product prices as well as of gas and petroleum surcharges/levies.

- $\quad$ Monthly data on the import parity prices as well as central depot prices of the six major oil products, within one month.

- Quarterly data on KESC and WAPDA loans and debt outstanding, within one month.

- Upon the adoption of the plan for the elimination of inter-corporate circular debt, monthly reports on inter-corporate circular debt will be reported within one month.

- Information on new liabilities incurred or guaranteed by the government in settling the circular-debt, including government guarantees of Term Financing Certificates (TFCs) issued by Pakistan Electric Power Company (PEPCO). The information will include the size of the government exposure, the duration of the guarantee or claim, and any other provisions relevant for the government's exposure.

- Information on the transactions between the Ministry of Finance and the SBP with regard to the use of Fund resources for budget purposes on monthly basis. 
Table 1. Pakistan: Budgetary Support 1/

(In million of rupees)

\begin{tabular}{|c|c|c|c|c|c|c|}
\hline & & 30-Jun-08 & 30-Sep-08 & 31-Dec-08 & 31-Mar-09 & 30-May-09 \\
\hline A. & Central Government & $1,577,064$ & $1,693,872$ & $1,816,104$ & $1,829,279$ & $1,901,105$ \\
\hline \multirow[t]{4}{*}{1.} & Scheduled Banks & 509,710 & 397,816 & 504,189 & 649,648 & 683,468 \\
\hline & a) Government Securities & 173,171 & 176,905 & 172,547 & 199,441 & 214,048 \\
\hline & b) Treasury Bills & 559,825 & 476,236 & 560,584 & 666,477 & 710,532 \\
\hline & c) Government Deposits & $-223,286$ & $-255,324$ & $-228,942$ & $-216,269$ & $-241,112$ \\
\hline \multirow[t]{14}{*}{2.} & State Bank & $1,067,354$ & $1,296,056$ & $1,311,916$ & $1,179,630$ & $1,217,637$ \\
\hline & a) Government Securities & 3,167 & 3,168 & 3,168 & 3,148 & 3,148 \\
\hline & b) Accrued Profit on MRTBs & 18,200 & 33,855 & 34,500 & 44,031 & 41,117 \\
\hline & c) Treasury Bills & $1,053,122$ & $1,256,910$ & $1,312,622$ & $1,227,858$ & $1,209,858$ \\
\hline & MTFBs purchased for replenishment of cash balances & $1,036,610$ & $1,241,198$ & $1,296,910$ & $1,212,149$ & $1,200,349$ \\
\hline & d) Adhoc Treasury Bills & & 0 & & & 0 \\
\hline & e) Ways and Means Advances & & 0 & & & 0 \\
\hline & f) Treasury Currency & 8,152 & 8,150 & 8,153 & 8,153 & 8,153 \\
\hline & g) Debtor Balances (Excl. Zakat Fund) & 0 & 0 & 0 & 0 & 0 \\
\hline & $\begin{array}{l}\text { h) Government Deposits } \\
\text { (Excl. Zakat and Privatization Fund) }\end{array}$ & $-20,748$ & $-11,490$ & $-51,990$ & $-109,023$ & $\begin{array}{r}-50,102 \\
0\end{array}$ \\
\hline & i) Special Account-Debt Repayment & 0 & 0 & & & 0 \\
\hline & i) Payment to HBL on a/c of HC\&EB & -287 & -287 & -287 & -287 & -287 \\
\hline & j) Adjustment for use of Privatization Proceeds & & & & & \\
\hline & for Debt Retirement & 5,749 & 5,749 & 5,749 & 5,749 & 5,749 \\
\hline \multirow{5}{*}{$\begin{array}{l}\text { B. } \\
1 .\end{array}$} & Provincial Governments & $-212,460$ & $-208,026$ & $-241,207$ & $-259,040$ & $-226,115$ \\
\hline & Scheduled Banks $(a+b-c)$ & $-178,821$ & $-172,430$ & $-185,836$ & $-194,616$ & $-201,157$ \\
\hline & a) Government Securities & 76 & 76 & 37 & 0 & 0 \\
\hline & b) Advances to Punjab Government for Cooperatives & 1,024 & 1,024 & 1,024 & 1,024 & 1,024 \\
\hline & c) Government Deposits & $-179,921$ & $-173,530$ & $-186,897$ & $-195,640$ & $-202,181$ \\
\hline \multirow[t]{4}{*}{2.} & State Bank & $-33,639$ & $-35,596$ & $-55,371$ & $-64,424$ & $-24,958$ \\
\hline & a) Debtor Balances (Excl. Zakat Fund) & 18,719 & 14,837 & 15,588 & 16,366 & 29,844 \\
\hline & b) Ways and Means Advances & 0 & 0 & & & 0 \\
\hline & c) Government Deposits (Excl.Zakat Fund) & $-52,357$ & $-50,433$ & $-70,958$ & $-80,790$ & $-54,803$ \\
\hline \multirow[t]{2}{*}{ C } & Net Govt. Budgetary Borrowings & & & & & \\
\hline & from the Banking system & $1,364,604$ & $1,485,846$ & $1,574,898$ & $1,570,239$ & $1,674,990$ \\
\hline \multirow[t]{10}{*}{ D } & Through SBP & $1,033,715$ & $1,260,460$ & $1,256,545$ & $1,115,206$ & $1,192,679$ \\
\hline & Through Scheduled Banks & 330,888 & 225,386 & 318,353 & 455,033 & 482,311 \\
\hline & Memorandum Items & & & & & \\
\hline & 1 Accrued Profit on SBP holding of MRTBs & 18,200 & 33,855 & 34,500 & 44,031 & 41,117 \\
\hline & 2 Scheduled banks ' deposits of Privatization Commission & $-1,815$ & $-2,487$ & $-1,097$ & $-2,372$ & $-2,377$ \\
\hline & $\begin{array}{l}\text { Outstanding amount of MTBs (Primary market; } \\
3 \text { discounted value) }\end{array}$ & 536,977 & 451,756 & 525,438 & 639,450 & 688,793 \\
\hline & Net Govt. Borrowings (Cash basis) & & & & & \\
\hline & From Banking System, of which & $1,325,371$ & $1,429,999$ & $1,506,349$ & $1,501,553$ & $1,614,511$ \\
\hline & From SBP & $1,015,516$ & $1,226,605$ & $1,222,045$ & $1,071,175$ & $1,151,561$ \\
\hline & From Scheduled Banks & 309,855 & 203,394 & 284,304 & 430,378 & 462,949 \\
\hline
\end{tabular}

Source: State Bank of Pakistan.

1/ Provisional data. 
Table 2. Pakistan: External Financing For Budget for 2008/09 and 2009/10 (in millions of US dollars)

\begin{tabular}{|c|c|c|c|c|c|c|c|c|c|c|}
\hline & \multicolumn{5}{|c|}{$2008 / 09$} & \multicolumn{5}{|c|}{$2009 / 10$} \\
\hline & $\begin{array}{l}\text { Jul.-Sep. } \\
\text { Act. }\end{array}$ & $\begin{array}{l}\text { Oct.-Dec. } \\
\text { Act. }\end{array}$ & $\begin{array}{l}\text { Jan.-Mar. } \\
\text { Act. }\end{array}$ & $\begin{array}{l}\text { Apr.-Jun. } \\
\text { Proj. }\end{array}$ & $\begin{array}{l}\text { Total } \\
\text { Proj. }\end{array}$ & $\begin{array}{l}\text { Jul.-Sep. } \\
\text { Proj. }\end{array}$ & $\begin{array}{l}\text { Oct.-Dec. } \\
\text { Proj. }\end{array}$ & $\begin{array}{l}\text { Jan.-Mar. } \\
\text { Proj. }\end{array}$ & $\begin{array}{l}\text { Apr.-Jun. } \\
\text { Proj. }\end{array}$ & $\begin{array}{l}\text { Total } \\
\text { Proj. }\end{array}$ \\
\hline Project Aid & 248 & 331 & 279 & 316 & 1,174 & 673 & 885 & 946 & 938 & 3,442 \\
\hline Grants, of which & 23 & 21 & 61 & 36 & 141 & 252 & 222 & 223 & 43 & 740 \\
\hline Tokyo pledge & & & & & & 142 & 200 & 200 & 20 & 562 \\
\hline IDP grants & & & & & & 110 & 73 & 18 & 18 & 220 \\
\hline Earth Quake grants & 1 & 2 & 3 & 7 & 13 & 6 & 8 & 10 & 15 & 39 \\
\hline World Bank, of which & 61 & 56 & 42 & 60 & 218 & 45 & 45 & 45 & 395 & 530 \\
\hline Tokyo pledge & & & & & & 0 & 0 & 0 & 150 & 150 \\
\hline Earthquake & 7 & 21 & 0 & 0 & 29 & 0 & 0 & 0 & 0 & 0 \\
\hline ADB, of which & 111 & 85 & 53 & 115 & 364 & 70 & 75 & 83 & 110 & 338 \\
\hline Tokyo pledge & & & & & & 0 & 0 & 0 & 20 & 20 \\
\hline Earthquake & 7 & 9 & 5 & 11 & 32 & 0 & 0 & 0 & 0 & 0 \\
\hline Other bilateral project loan, of which & 52 & 44 & 68 & 96 & 260 & 300 & 535 & 585 & 375 & 1,795 \\
\hline Tokyo pledge & & & & & & 200 & 435 & 485 & 238 & 1,358 \\
\hline Other Earthquake Loans & 0 & 0 & 0 & 2 & 2 & 0 & 0 & 0 & 0 & 0 \\
\hline Commodity Aid (Import of S.Arabia Urea) & 0 & 123 & 52 & 0 & 175 & 0 & 0 & 0 & 0 & 0 \\
\hline Program Loans & 494 & 811 & 677 & 812 & 2,794 & 400 & 300 & 445 & 1,600 & 2,745 \\
\hline World Bank & 0 & 0 & 485 & 221 & 706 & 300 & 0 & 0 & 300 & 600 \\
\hline$A D B$ & 494 & 100 & 96 & 542 & 1,232 & 0 & 0 & 145 & 1,100 & 1,245 \\
\hline Tokyo pledge, of which & 0 & 0 & 0 & 0 & & 0 & 200 & 200 & 0 & 400 \\
\hline World Bank & & & & & & 0 & 0 & 0 & 0 & 0 \\
\hline$A D B$ & & & & & & 0 & 0 & 100 & 0 & 100 \\
\hline IDB & 0 & 561 & 77 & 18 & 656 & 100 & 100 & 100 & 200 & 500 \\
\hline Short-term commercial & 0 & 150 & 19 & 31 & 200 & 0 & 0 & 0 & 0 & 0 \\
\hline Budgetary Grants & 40 & 30 & 56 & 0 & 126 & 25 & 38 & 62 & 0 & 125 \\
\hline Privatization & 0 & 0 & 0 & 0 & 0 & 0 & 133 & 133 & 0 & 266 \\
\hline GDRs & 0 & 0 & 0 & 0 & 0 & 0 & 0 & 0 & 0 & 0 \\
\hline Securitization/China & 0 & 0 & 500 & 0 & 500 & 0 & 0 & 0 & 0 & 0 \\
\hline IDP grants & & & & & & 110 & 73 & 18 & 18 & 0 \\
\hline Budget financing from the Fund & & & & & & 715 & 357 & 357 & $-1,430$ & 0 \\
\hline Amortization & 700 & 802 & 810 & 492 & 2,804 & 330 & 883 & 990 & 259 & 2,462 \\
\hline Medium and Long-term & 538 & 258 & 760 & 398 & 1,954 & 280 & 237 & 890 & 259 & 1,666 \\
\hline Euro bonds & 22 & 0 & 500 & 0 & 522 & 22 & 0 & 600 & 0 & 622 \\
\hline IDB $>1$ year & 200 & 0 & 0 & 0 & 200 & 0 & 0 & 0 & 0 & 0 \\
\hline Other & 316 & 258 & 260 & 398 & 1,232 & 258 & 237 & 290 & 259 & 1,044 \\
\hline Short-term & 162 & 544 & 50 & 94 & 850 & 50 & 646 & 100 & 0 & 796 \\
\hline IDB & 162 & 428 & 0 & 94 & 684 & 0 & 530 & 100 & 0 & 630 \\
\hline Commercial & 0 & 116 & 50 & 0 & 166 & 50 & 116 & 0 & 0 & 166 \\
\hline \multicolumn{11}{|l|}{$\begin{array}{l}\text { Memorandum items: } \\
\text { (cumulative from the beginning of fiscal year) }\end{array}$} \\
\hline Net budget financing & 82 & 452 & 1,153 & 1,789 & & 1,593 & 2,497 & 3,469 & 4,336 & \\
\hline $\begin{array}{l}\text { Net program financing for the budget (excl. Tokyo } \\
\text { related disbursements, IDP grants, Fund financing to the }\end{array}$ & & & & & & & & & & \\
\hline budget) & -166 & -127 & 296 & 616 & & 95 & -517 & $-1,067$ & 274 & \\
\hline Tokyo-related disbursements (excl. multilateral sources) & 0 & 0 & 0 & 0 & & 342 & 1,177 & 1,962 & 2,220 & \\
\hline Tokyo-related disbursements from multilateral sources & 0 & 0 & 0 & 0 & & 0 & 0 & 100 & 270 & \\
\hline Total budget grants for IDP & 0 & 0 & 0 & 0 & & 110 & 183 & 202 & 220 & \\
\hline Project grants & 24 & 47 & 111 & 154 & & 258 & 488 & 721 & 779 & \\
\hline SDR allocation & & & & & & 0 & 1,267 & 1,267 & 1,267 & \\
\hline
\end{tabular}

Sources: Pakistani authorities; and Fund staff projections. 
Table 3. Pakistan: Total Outstanding Domestic Debt

(In billion of rupees)

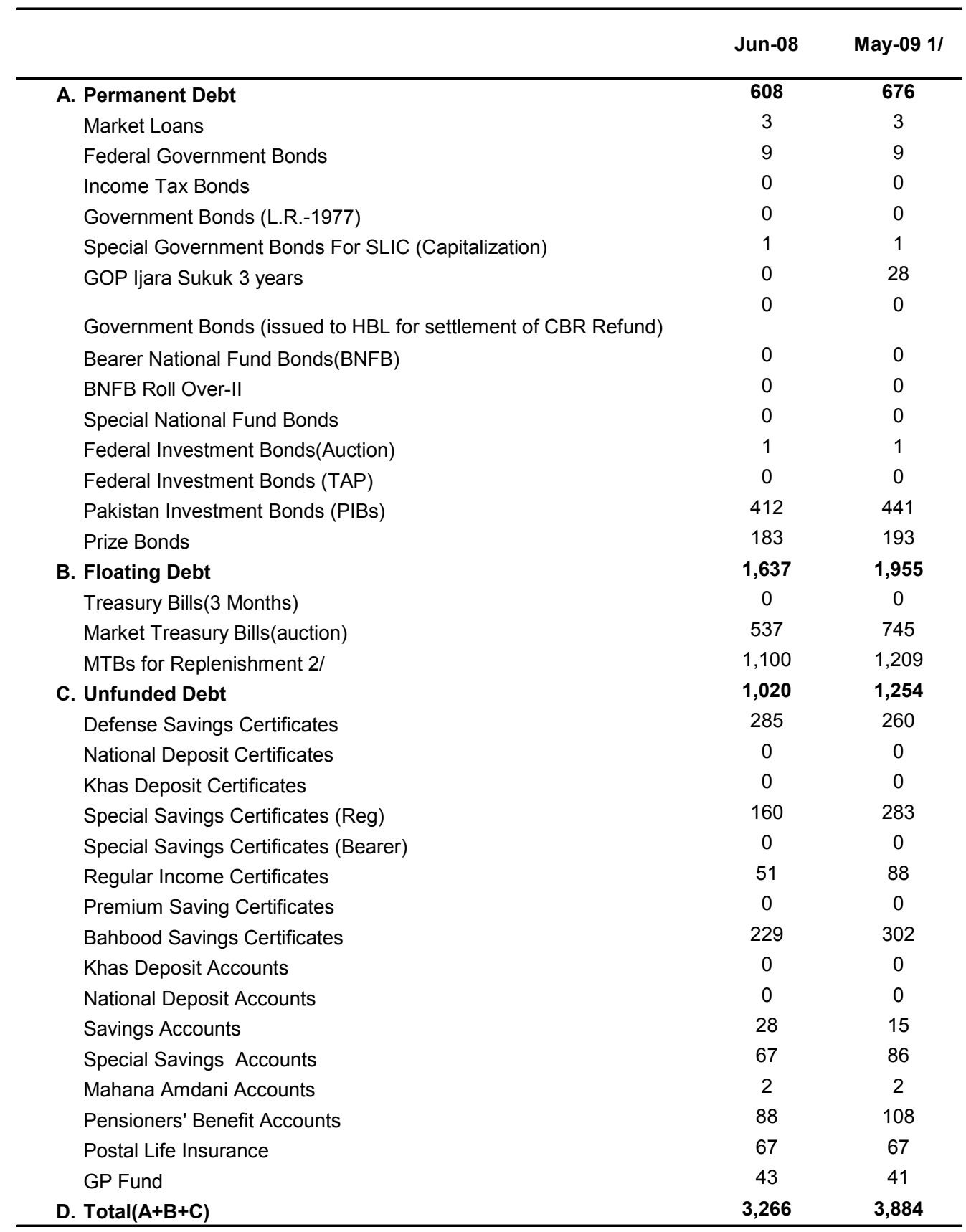

Source: State Bank of Pakistan.

1/ Provisional data.

2/ Inclusive of outright sale of MTBs to commercial banks. 


\section{INTERNATIONAL MONETARY FUND}

\section{PAKISTAN}

\section{Second Review and Request for the Augmentation of Access Under the Stand-By Arrangement \\ Informational Annex}

Prepared by Middle East and Central Asia Department

July 29, 2009

Contents

Page

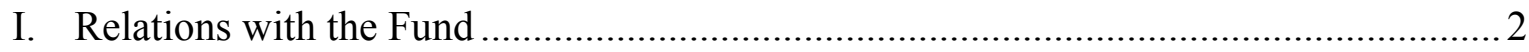

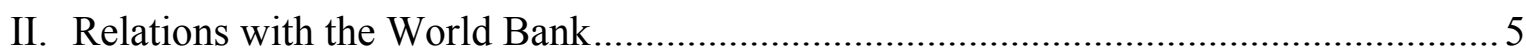




\section{Annex I. Pakistan: Relations With the Fund}

As of June 30, 2009

I. Membership Status: Joined: 07/11/1950; Article VIII

II. General Resources Account:

Quota

Fund Holdings of Currency

Reserve position in Fund

III. SDR Department:

Net cumulative allocation

Holdings

IV. Outstanding Purchases and Loans:

Stand-by Arrangements

PRGF arrangements

Extended arrangements $\underline{\text { SDR Million }}$

$1,033.70$

$3,669.52$

0.12

$\underline{\text { SDR Million }}$

169.99

98.41

$\underline{\text { SDR Million }}$

$2,635.94$

680.52

3.16
\% Quota

100.00

354.99

0.01

$\%$ Allocation

100.00

57.89

$\%$ Quota

255.00

65.83

0.31

V. Latest Financial Arrangements:

\begin{tabular}{|c|c|c|c|c|}
\hline Type & $\begin{array}{l}\text { Approval } \\
\text { Date }\end{array}$ & $\begin{array}{c}\text { Expiration } \\
\text { Date }\end{array}$ & $\begin{array}{l}\text { Amount Approved } \\
\text { (SDR Million) }\end{array}$ & $\begin{array}{l}\text { Amount Drawn } \\
\text { (SDR Million) }\end{array}$ \\
\hline Stand-By & $11 / 24 / 08$ & $10 / 23 / 2010$ & $5,168.50$ & $2,635.94$ \\
\hline PRGF & $12 / 06 / 2001$ & $12 / 05 / 2004$ & $1,033.70$ & 861.42 \\
\hline Stand-By & $11 / 29 / 2000$ & $09 / 30 / 2001$ & 465.00 & 465.00 \\
\hline
\end{tabular}

VI. Projected Payments to the Fund ${ }^{1,2}$

(SDR Million; based on existing use of resources and present holdings of SDRs):

\begin{tabular}{lrrrrr} 
& \multicolumn{5}{c}{ Forthcoming } \\
\cline { 2 - 6 } & $\mathbf{2 0 0 9}$ & $\mathbf{2 0 1 0}$ & $\mathbf{2 0 1 1}$ & $\mathbf{2 0 1 2}$ & $\mathbf{2 0 1 3}$ \\
Principal & 68.91 & 172.28 & 172.28 & $1,322.27$ & $1,404.11$ \\
Charges/Interest & 23.28 & 44.98 & 44.11 & 35.13 & 14.56 \\
Total & 92.19 & 217.26 & 216.40 & $1,357.41$ & $1,418.67$
\end{tabular}

A.

\section{Nonfinancial Relations}

\footnotetext{
${ }^{1}$ This schedule presents all currently scheduled payments to the IMF, including repayment expectations and repayment obligations. The IMF Executive Board can extend repayment expectations (within predetermined limits) upon request by the member if its external payment position is not strong enough to meet the expectations without undue hardship or risk.

${ }^{2}$ When a member has overdue financial obligations outstanding for more than three months, the amount of such arrears will be shown in this section.
} 


\section{Exchange System}

On May 19, 1999, the dual exchange rate system was unified, with all international transactions conducted at the interbank market exchange rate (FIBR). The Fund classifies Pakistan's exchange rate regime as floating. Pakistan has accepted the obligations of Article VIII. It maintains a restriction subject to Fund approval in the form of a 25 percent limit on advance payments for imports of goods. The authorities eliminated cash margin requirements on letters of credit in June 2009.

\section{Last Article IV Consultation}

The last Article IV consultation (Country Report 09/123) was discussed by the Executive Board on March 23, 2009.

\section{Safeguards Assessments}

In accordance with the Fund's safeguard assessment policy, the State Bank of Pakistan (SBP) was subject to a full safeguards assessment under the Stand-By Arrangement that was approved in November 2008. This update assessment included a safeguard mission to the SBP in late January 2009. The assessment found improved transparency of the audited financial statements, coupled with stronger governance oversight by the Central Board and the Audit Committee. The SBP embarked on a modernization program that saw the implementation of a new organization structure, IT systems, and the recruitment of a qualified professional staff. However, prompt action is needed to safeguard central bank independence via amending the SBP Act, and expedite its transition to the inter-agency group formed by the authorities to facilitate its enactment. Also, staff noted the need to strengthen controls over program data reporting to the Fund through an independent review mechanism.

\section{FSAP Participation and ROSCs}

\begin{tabular}{|l|c|l|}
\hline Fiscal Transparency Module & $11 / 28 / 2000$ & (www.imf.org) \\
\hline Fiscal Transparency Module-Update & $11 / 22 / 2004$ & Country Report No. 04/416 \\
\hline $\begin{array}{l}\text { Financial System Stability Assessment } \\
\text { Financial Sector Assessment Program }\end{array}$ & $6 / 23 / 2004$ & Country Report No. 04/215 \\
\hline $\begin{array}{l}\text { Data Module and Detailed Assessment } \\
\text { Using Quality Assessment Framework }\end{array}$ & $11 / 29 / 2004$ & Country Report No. 04/398 \\
\hline $\begin{array}{l}\text { Data Module, Reassessment of Monetary } \\
\text { Statistics and Detailed Assessment Using } \\
\text { Quality Assessment Framework }\end{array}$ & $2 / 2 / 2007$ & Country Report No. 07/74 \\
\hline
\end{tabular}




\begin{tabular}{|l|c|l|}
\hline $\begin{array}{l}\text { Fiscal Transparency Module—Draft } \\
\text { Update }\end{array}$ & $2 / 13 / 2007$ & Country Report No. 08/129 \\
\hline $\begin{array}{l}\text { Financial System Stability Assessment, } \\
\text { Financial Sector Assessment Program } \\
\text { Update }\end{array}$ & $9 / 22 / 2008$ & In progress \\
\hline
\end{tabular}

\section{Recent Technical Assistance}

\section{FAD}

January 2002: Fiscal data management, quality, and transparency.

January 2003: Tax administration.

February/March 2003: Customs administration.

April 2004: Fiscal reporting.

April 2007: Public financial management.

\section{MCM}

November/December 2004: Public debt reform and capacity building program (joint with World Bank).

March/April 2005: Development of the Insurance Sector.

December 2006: Monetary policy framework.

April 2007: Monetary policy framework, the SBP's balance sheet, and the Banking Services Corporation.

\section{STA}

February 2002: External sector statistics/SDDS subscription.

April/May 2005: National accounts and consumer price statistics.

May 2007: Statistics on the international investment position.

\section{LEG}

July 2008: Deposit Protection Fund.

July 2008: Central Bank Law.

August 2008: Banking Law.

\section{Resident Representative}

A resident representative has been stationed in Islamabad since August 1991. 


\section{AnNex II. Pakistan: Relations With the World Bank Group}

Thomas Buckley, Senior Country Officer, Telephone: (202) 473-0075

1. Pakistan is among the largest recipients of World Bank financial assistance. In FY2007, World Bank support to Pakistan totaled \$985 million, making it the seventh largest borrower in the world. The World Bank Group program in Pakistan consists of an integrated package of financial support, including IBRD lending, concessional IDA credits and IFC investments, along with complementary analytical and advisory services.

2. The Bank Group's Board of Directors endorsed a new Country Assistance Strategy (CAS) for Pakistan on June 1, 2006, covering fiscal years 2006 through 2009. The CAS outlines the Bank's strategic approach to helping Pakistan achieve its development goals over a four-year period. Despite recent good economic performance, Pakistan's development challenges remain formidable. The strategy addresses the key challenge of sustaining rapid growth in order to further reduce poverty. This will require accelerating human development so that the poor can participate in and benefit from growth. Sustained growth will also require sound macroeconomic management along with improvements in the investment climate, including regulatory reform and significant investment in infrastructure.

3. The CAS is designed around three main pillars:

- $\quad$ Sustained Growth and Improved Competitiveness: The principal focus of this pillar will be to support investments and reforms needed to improve the business environment for trade and investment and sustain rapid, private sector-led growth. The Bank will provide support to key sectors such as agriculture and infrastructure, and help the government strengthen macroeconomic management through improving public expenditures and supporting ongoing tax reforms.

- $\quad$ Strengthened Governance and Service Delivery: Priorities in this area will be to support further reforms and investment to increase efficiency, transparency, and accountability in the use of public resources, while supporting cross-cutting reforms needed to improve service delivery at all levels of government — with particular attention to health, education, water and sanitation, safety nets, and municipal services.

- Improved Lives and Protection of the Vulnerable: The Bank will focus on increased investment in the education and health sectors to help build the skilled, healthy work force needed to sustain strong growth. This area of the CAS also features targeted interventions to help the poor, including strengthened safety nets and targeted interventions and community-based approaches in rural areas. 
4. In line with Pakistan's recent performance and the government's request for increased Bank Group support, the CAS outlines a substantial increase in the volume of lending to Pakistan through FY2009. The expansion in lending will take place primarily in infrastructure (mainly in energy, water, and transport) and human development. One priority will be to continue to assist in addressing the impact of the October 2005 earthquake - up to $\$ 1$ billion will be used to support reconstruction and recovery. Overall, a flexible lending program of up to $\$ 6.5$ billion is set forth in the CAS. About half of this amount ( $\$ 3.1$ billion) will take the form of IDA credits. Policy-based lending will account for up to half of planned financial support.

5. The IFC strategy in Pakistan, as expressed in the CAS, seeks to increase investments with a target range of \$500-600 million during the FY2006-09 period. IFC activity will focus on three main sectors: financial, SME and infrastructure. IFC has also initiated a substantial TA program in Pakistan to build capacity and address constraints of the SME, infrastructure and financial sectors.

\section{IBRD/IDA financial operations since FY2002 are summarized below:}

Pakistan: World Bank Group Financial Operations

(In millions of U.S. dollars)

\begin{tabular}{|c|c|c|c|c|c|c|}
\hline & FY2002 & FY2003 & FY2004 & FY2005 & FY2006 & FY2007 \\
\hline \multicolumn{7}{|c|}{ Commitments } \\
\hline IBRD & $\ldots$ & $\ldots$ & 50 & 347 & 315 & 100 \\
\hline IDA & 800 & 297 & 731 & 500 & 1,161 & 885 \\
\hline \multicolumn{7}{|c|}{ Disbursements } \\
\hline IBRD & 38 & 40 & 13 & 203 & 149 & 154 \\
\hline IDA & 830 & 316 & 290 & 778 & 1,061 & 1,030 \\
\hline \multicolumn{7}{|c|}{ Repayments } \\
\hline IBRD & 255 & 257 & 277 & 299 & 289 & 265 \\
\hline IDA & 71 & 83 & 96 & 111 & 117 & 170 \\
\hline \multicolumn{7}{|c|}{ Debt Disbursed and Outstanding } \\
\hline IBRD & 2,820 & 2,706 & 2,526 & 2,406 & 2,247 & 2,132 \\
\hline IDA & 5,097 & 5,604 & 6,020 & 6,651 & 7,627 & 8,700 \\
\hline
\end{tabular}




\title{
INTERNATIONAL MONETARY FUND
}

\section{Pakistan-Update of Assessment of the Risks to the Fund and the Fund's Liquidity Position}

\author{
Prepared by the Finance and Strategy, Policy, and Review Departments \\ In consultation with other Departments \\ Approved by Andrew Tweedie and Michele Shannon
}

August 4, 2009

1. This paper updates the assessment of the risks to the Fund and the effect on the Fund's liquidity position arising from the Stand-By Arrangement (SBA) for Pakistan in view of its proposed augmentation. ${ }^{1}$ The authorities are requesting an augmentation of access of about SDR 2.1 billion (200 percent of quota) under the current 23-month SBA approved on November 24, 2008, of which some SDR 0.95 billion (92 percent of quota) would be used to provide budget financing before donor pledges are disbursed. Total access would rise to about SDR 7.2 billion (700 percent of quota). The augmentation is to be combined with (i) a rephasing of purchases, and (ii) an extension of the arrangement through end-2010 and a change in the dates of availability, such that access in the balance of 2009 and 2010 would increase and be phased evenly for the remainder of the program (Table 1).

Table 1. Pakistan: Proposed Augmentation under the SBA-Access and Phasing

\begin{tabular}{|c|c|c|c|c|c|c|c|}
\hline \multirow[b]{3}{*}{ Availability } & \multirow[b]{3}{*}{ Date } & & & \multicolumn{4}{|c|}{ Percent of quota } \\
\hline & & \multicolumn{2}{|c|}{ SDR $\mathrm{mn}$} & \multicolumn{2}{|c|}{ Purchase } & \multicolumn{2}{|c|}{ Cumulative } \\
\hline & & Original & Proposed & Original & Proposed & Original & Proposed \\
\hline 2008 & November & $2,067.40$ & $2,067.40$ & 200.0 & 200.0 & 200.0 & 200.0 \\
\hline \multirow[t]{5}{*}{2009} & $\begin{array}{l}\text { March 1/ } \\
\text { June }\end{array}$ & $\begin{array}{l}568.54 \\
568.54\end{array}$ & 568.54 & $\begin{array}{l}55.0 \\
55.0\end{array}$ & 55.0 & $\begin{array}{l}255.0 \\
310.0\end{array}$ & 255.0 \\
\hline & August (approval) & & 766.70 & & 74.2 & & 329.2 \\
\hline & September & 434.15 & & 42.0 & & 352.0 & \\
\hline & November & & 766.70 & & 74.2 & & 403.3 \\
\hline & December & 434.15 & & 42.0 & & 394.0 & \\
\hline \multirow[t]{8}{*}{2010} & February & & 766.70 & & 74.2 & & 477.5 \\
\hline & March & 434.15 & & 42.0 & & 436.0 & \\
\hline & May & & 766.70 & & 74.2 & & 551.7 \\
\hline & June & 434.15 & & 42.0 & & 478.0 & \\
\hline & August & & 766.70 & & 74.2 & & 625.9 \\
\hline & September & 227.41 & & 22.0 & & 500.0 & \\
\hline & November & & 766.47 & & 74.1 & & 700.0 \\
\hline & Total & $5,168.50$ & $7,235.90$ & 500.0 & 700.0 & 500.0 & 700.0 \\
\hline
\end{tabular}

Source: Finance Department.

1/ The first review was completed on March 30 and the purchase was made on April 1.

\footnotetext{
${ }^{1}$ See Pakistan-Assessment of the Risks to the Fund and the Fund's Liquidity Position, IMF Country Report No 08/364, December 2008). Under the proposed augmentation, access would exceed the new annual and cumulative access limits adopted on March 24, indicating that an updated assessment is appropriate.
} 


\section{The Augmented ACCess Under the StAnd-By ARRANGement-Risks AND IMPACT ON FUND'S FINANCES}

\section{Under the proposed augmentation, the Fund would become a major source of external financing:}

- $\quad$ Pakistan's outstanding use of GRA resources started with an initial purchase of 200 percent of quota (SDR 2.1 billion) in November 2008. If all purchases were made as available under the proposed augmentation, Pakistan's GRA credit would peak at 700 percent of quota (SDR 7.2 billion) by November 2010. In terms of quota, this projected peak exposure would be above the median for recent exceptional access cases, represented by Pakistan's arrangement before the proposed augmentation (Figure 1). Including outstanding PRGF loans, the peak exposure would be 744 percent of quota (SDR 7.7 billion) also in November 2010.

\section{Figure 1. Fund Credit Outstanding in the GRA around Peak Borrowing 1/}

(In percent of quota)

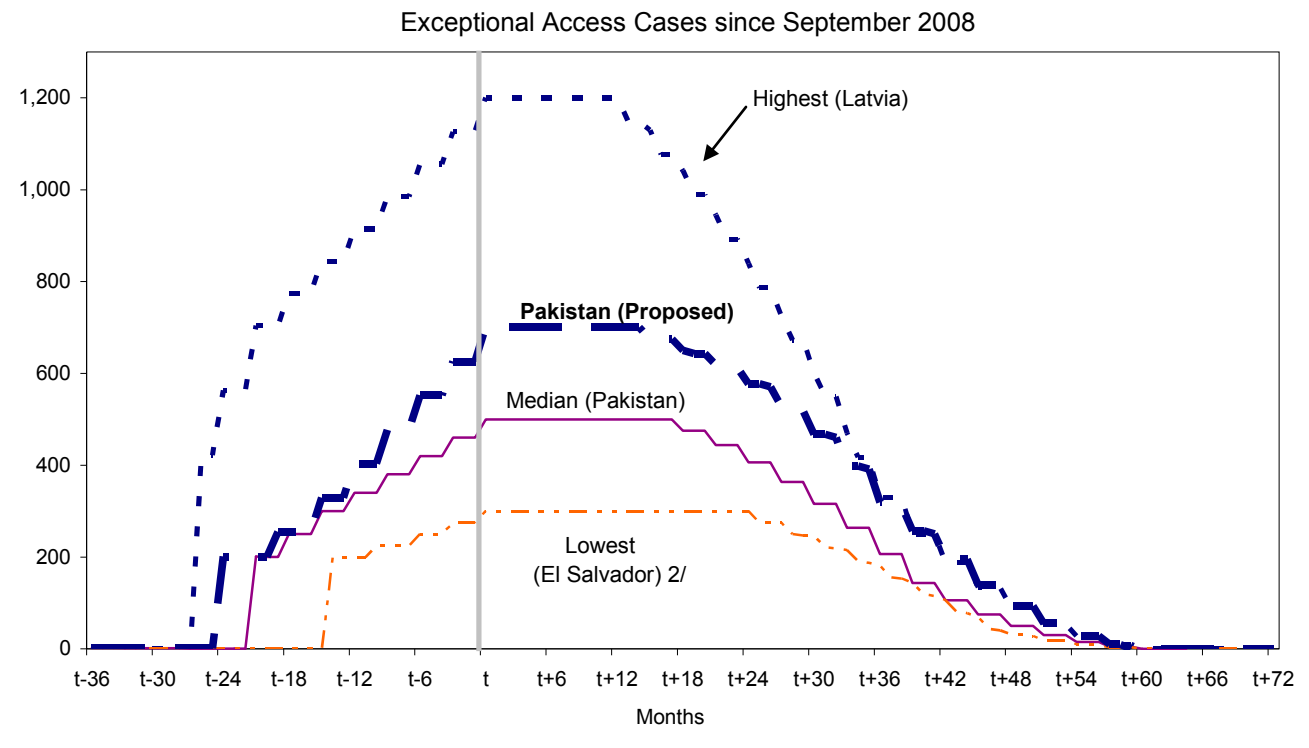

Source: IFS, Finance Department, and IMF staff estimates.

$1 /$ Peak borrowing ' $t$ ' is defined as the highest level of credit outstanding for a member. Repurchases are assumed to be on an obligations basis.

2/ The authorities have expressed their intention to treat the arrangement as precautionary, as balance of payments pressures have not materialized.

- $\quad$ Under the proposed augmentation, Pakistan's GRA credit would peak at 16.8 percent of total external debt in 2010/11, the second highest among recent exceptional access cases (Table 2 and Figure 2). This in part reflects the fact that Pakistan's external debt is relatively moderate versus comparators, reaching about 35 percent of GDP in $2010 / 11$.

- $\quad$ GRA credit outstanding to Pakistan would reach 6 percent of GDP and about 72 percent of gross international reserves by end of fiscal year 2010/11 (Table 2). Although, the peak relative to GDP would be slightly lower than the median of the 
recent exceptional access cases, the peak relative to gross international reserves would remain the highest among this group (Figure 2).

3. The proposed augmentation would bring Pakistan's debt service to the Fund to high levels relative to its debt service capacity and other exceptional access cases (Table 2 and Figure 2): ${ }^{2}$

- $\quad$ At its 2013/14 peak, public external debt service would reach 60 percent of gross international reserves, 25 percent of exports of goods and services, and 25 percent of tax revenues (excluding grants).

- Debt service to the GRA would peak in 2013/14 at 15 percent of exports of goods and services, the highest among the recent exceptional access cases reflecting in part the low level of exports in relation to GDP, and would account for more than half of total external debt service, which again partly reflects the relatively moderate overall external debt.

- Debt service due on GRA credit would peak at 15 percent of tax revenues (excluding grants) in 2013/14, and it would average 12 percent between 2012/13 and 2014/15, potentially imposing a significant burden on public finances.

\section{The impact on the Fund's finances of the augmentation would be relatively} moderate, although the overall impact of the Pakistan program remains significant (Table 3 ). The augmentation would reduce the one-year forward commitment capacity (FCC) by 2.3 percent. Following the August drawing, Pakistan would remain the fourth largest borrower, accounting for about 14 percent of total GRA credit outstanding. With precautionary balances currently at about SDR 7 billion, the ratio of Pakistan's GRA credit outstanding to precautionary balances would rise to just under 50 percent if the proposed August purchase is made; after the augmentation total access would rise above the current level of precautionary balances.

\footnotetext{
${ }^{2}$ Debt service to the Fund is calculated assuming that all purchases and repurchases are made as scheduled and surcharges are calculated according to the current schedule. The new system of surcharges will go into effect on August 1, 2009, subject to grandfathering at the member's request. Our calculations assume the same assumptions as in the staff report, which is that the authorities adopt the new system.
} 
Table 2. Pakistan-Capacity to Repay Indicators 1/

\begin{tabular}{|c|c|c|c|c|c|c|c|c|}
\hline & Aug-09 & 2010 & 2011 & 2012 & 2013 & 2014 & 2015 & 2016 \\
\hline \multicolumn{9}{|c|}{ Exposure and Repayments (In SDR millions) } \\
\hline $\begin{array}{l}\text { GRA credit to Pakistan } \\
\text { (In percent of quota) } \\
\text { Charges due on GRA credit 2/ } \\
\text { Debt service due on GRA credit 2/ }\end{array}$ & $\begin{array}{l}3,402.6 \\
(329.2)\end{array}$ & $\begin{array}{r}5,702.6 \\
(551.7) \\
85.1 \\
85.1\end{array}$ & $\begin{array}{r}7,235.9 \\
(700.0) \\
167.0 \\
167.0\end{array}$ & $\begin{array}{r}6,648.0 \\
(643.1) \\
183.2 \\
771.1\end{array}$ & $\begin{array}{r}4,826.1 \\
(466.9) \\
165.3 \\
1,987.2\end{array}$ & $\begin{array}{r}2,012.5 \\
(194.7) \\
77.4 \\
2,891.0\end{array}$ & $\begin{array}{r}287.5 \\
(27.8) \\
18.9 \\
1,743.9\end{array}$ & $\begin{array}{r}0.0 \\
(0.0) \\
2.2 \\
289.7\end{array}$ \\
\hline \multicolumn{9}{|l|}{ Debt and Debt Service Ratios 3/ } \\
\hline $\begin{array}{l}\text { In percent of GDP } \\
\text { Total external debt } \\
\text { External debt, public } \\
\text { GRA credit to Pakistan } \\
\text { Total external debt service } \\
\text { Public external debt service } \\
\text { Debt service due on GRA credit }\end{array}$ & $\begin{array}{r}29.9 \\
28.0 \\
3.1 \\
3.1 \\
2.1\end{array}$ & $\begin{array}{r}32.1 \\
30.2 \\
4.8 \\
2.6 \\
1.7 \\
0.1\end{array}$ & $\begin{array}{r}35.1 \\
33.1 \\
5.9 \\
2.0 \\
1.7 \\
0.1\end{array}$ & $\begin{array}{r}35.5 \\
33.5 \\
5.1 \\
2.3 \\
2.0 \\
0.6\end{array}$ & $\begin{array}{r}34.5 \\
32.5 \\
3.5 \\
3.1 \\
2.8 \\
1.4\end{array}$ & $\begin{array}{r}32.5 \\
30.8 \\
1.4 \\
3.5 \\
3.3 \\
2.0\end{array}$ & $\begin{array}{r}30.7 \\
29.1 \\
0.2 \\
2.6 \\
2.4 \\
1.1\end{array}$ & $\begin{array}{r}29.5 \\
28.0 \\
0.0 \\
1.9 \\
1.5 \\
0.2\end{array}$ \\
\hline $\begin{array}{l}\text { In percent of Gross International Re } \\
\text { Total external debt } \\
\text { External debt, public } \\
\text { GRA credit to Pakistan } \\
\text { Total external debt service } \\
\text { Public external debt service } \\
\text { Debt service due on GRA credit }\end{array}$ & $\begin{array}{r}547.1 \\
512.2 \\
56.0 \\
56.3 \\
38.0\end{array}$ & $\begin{array}{r}426.1 \\
400.4 \\
63.5 \\
34.0 \\
22.2 \\
0.9\end{array}$ & $\begin{array}{r}428.6 \\
404.0 \\
72.0 \\
24.2 \\
20.8 \\
1.7\end{array}$ & $\begin{array}{r}473.4 \\
446.8 \\
68.4 \\
30.2 \\
26.6 \\
7.9\end{array}$ & $\begin{array}{r}542.7 \\
512.7 \\
55.4 \\
48.5 \\
44.5 \\
22.8\end{array}$ & $\begin{array}{r}590.8 \\
558.9 \\
25.0 \\
63.9 \\
59.5 \\
35.9\end{array}$ & $\begin{array}{r}523.3 \\
495.9 \\
3.1 \\
44.7 \\
40.9 \\
19.0\end{array}$ & $\begin{array}{r}467.2 \\
443.7 \\
0.0 \\
30.7 \\
24.2 \\
2.7\end{array}$ \\
\hline $\begin{array}{l}\text { In percent of Exports of Goods and } \\
\text { Total external debt service } \\
\text { Public external debt service } \\
\text { Debt service due on GRA credit }\end{array}$ & $\begin{array}{l}22.4 \\
15.1\end{array}$ & $\begin{array}{r}19.9 \\
13.0 \\
0.6\end{array}$ & $\begin{array}{r}15.7 \\
13.5 \\
1.1\end{array}$ & $\begin{array}{r}18.0 \\
15.8 \\
4.7\end{array}$ & $\begin{array}{l}24.2 \\
22.1 \\
11.3\end{array}$ & $\begin{array}{l}27.3 \\
25.4 \\
15.3\end{array}$ & $\begin{array}{r}20.3 \\
18.6 \\
8.6\end{array}$ & $\begin{array}{r}15.0 \\
11.9 \\
1.3\end{array}$ \\
\hline $\begin{array}{l}\text { In percent of Tax Revenues (excludi } \\
\text { Public external debt service } \\
\text { Debt service due on GRA credit }\end{array}$ & 20.4 & $\begin{array}{r}15.7 \\
0.7\end{array}$ & $\begin{array}{r}14.9 \\
1.2\end{array}$ & $\begin{array}{r}16.4 \\
4.9\end{array}$ & $\begin{array}{l}22.2 \\
11.4\end{array}$ & $\begin{array}{l}25.1 \\
15.2\end{array}$ & $\begin{array}{r}18.3 \\
8.5\end{array}$ & $\begin{array}{r}11.7 \\
1.3\end{array}$ \\
\hline $\begin{array}{l}\text { In percent of Total External Debt } \\
\text { GRA credit to Pakistan }\end{array}$ & 10.2 & 14.9 & 16.8 & 14.5 & 10.2 & 4.2 & 0.6 & 0.0 \\
\hline $\begin{array}{l}\text { In percent of Total External Debt Se } \\
\text { Debt service due on GRA credit }\end{array}$ & & 2.8 & 6.9 & 26.3 & 47.0 & 56.2 & 42.5 & 8.9 \\
\hline $\begin{array}{l}\text { In percent of Total Public External D } \\
\text { GRA credit to Pakistan } \\
\text { Total debt to Fund }\end{array}$ & $\begin{array}{l}10.9 \\
13.1\end{array}$ & $\begin{array}{l}15.9 \\
17.3\end{array}$ & $\begin{array}{l}17.8 \\
18.7\end{array}$ & $\begin{array}{l}15.3 \\
15.8\end{array}$ & $\begin{array}{l}10.8 \\
11.0\end{array}$ & $\begin{array}{l}4.5 \\
4.5\end{array}$ & $\begin{array}{l}0.6 \\
0.6\end{array}$ & $\begin{array}{l}0.0 \\
0.0\end{array}$ \\
\hline $\begin{array}{l}\text { In percent of Total Public External D } \\
\text { Debt service due on GRA credit } \\
\text { Total debt service to Fund }\end{array}$ & & $\begin{array}{r}4.3 \\
12.2\end{array}$ & $\begin{array}{r}8.0 \\
16.3\end{array}$ & $\begin{array}{l}29.8 \\
36.2\end{array}$ & $\begin{array}{l}51.3 \\
54.4\end{array}$ & $\begin{array}{l}60.4 \\
61.5\end{array}$ & $\begin{array}{l}46.5 \\
46.9\end{array}$ & $\begin{array}{l}11.3 \\
11.3\end{array}$ \\
\hline
\end{tabular}

Sources: Pakistan authorities, Finance Department, World Economic Outlook, and IMF staff estimates.

1/ Assumes full drawings. Pakistan's fiscal year ends on June 30. All data are expressed as the end-June projection of the corresponding years.

2/ Includes surcharges and service fees.

3/ Staff projections for external debt, GDP, gross international reserves, and exports of goods and services, as used in the staff report that requests the proposed

augmentation of the SBA. For August 2009, projections for external debt, GDP, gross international reserves, and exports of goods and services are for end-June 2009. 
Figure 2. Peak Fund Exposure and Debt Service Ratios for Recent Exceptional Access Cases

Peak Fund Exposure Ratios

A. In Percent of GDP

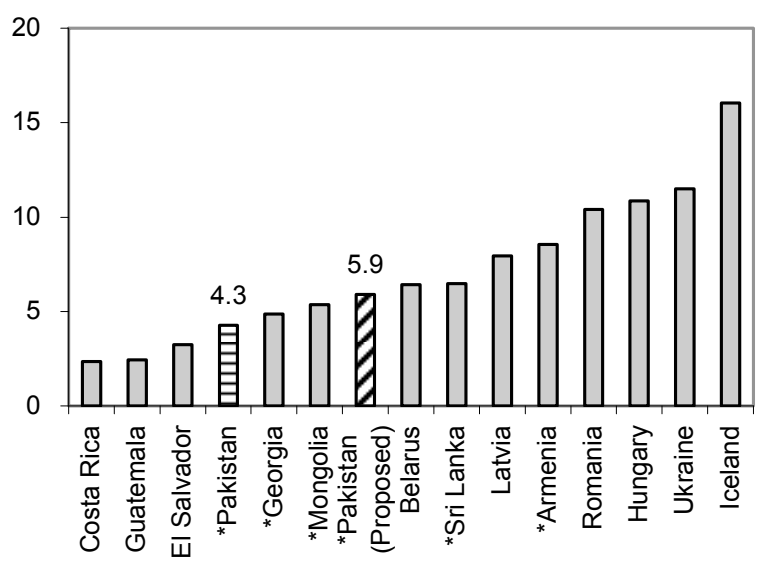

B. In Percent of Gross International Reserves

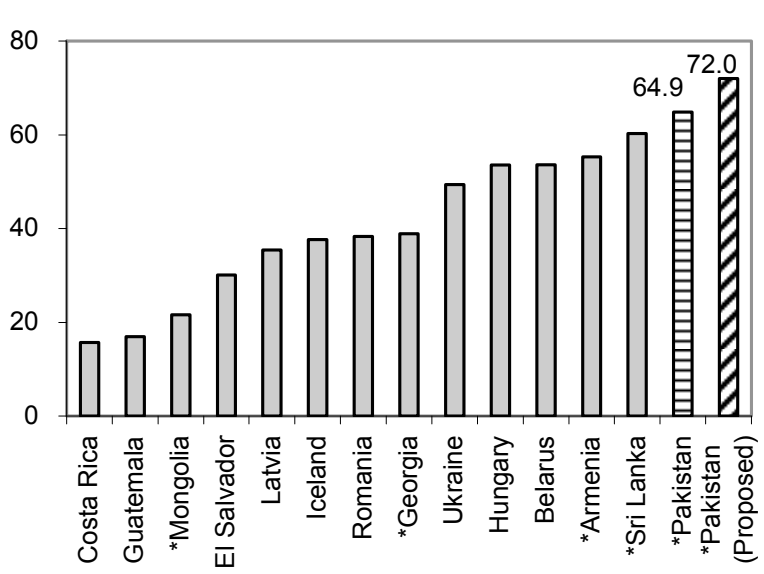

C. In Percent of Total External Debt

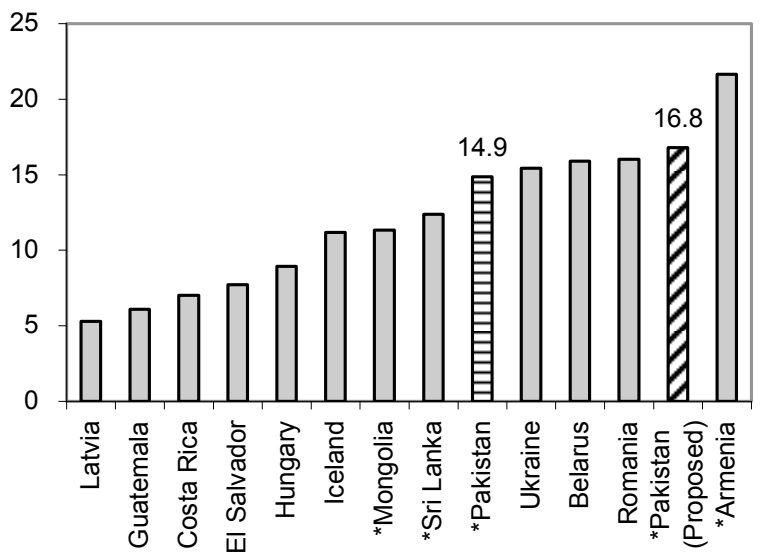

Peak Debt Service Ratios

A. Total External Debt Service in Percent of Exports of Goods and Services

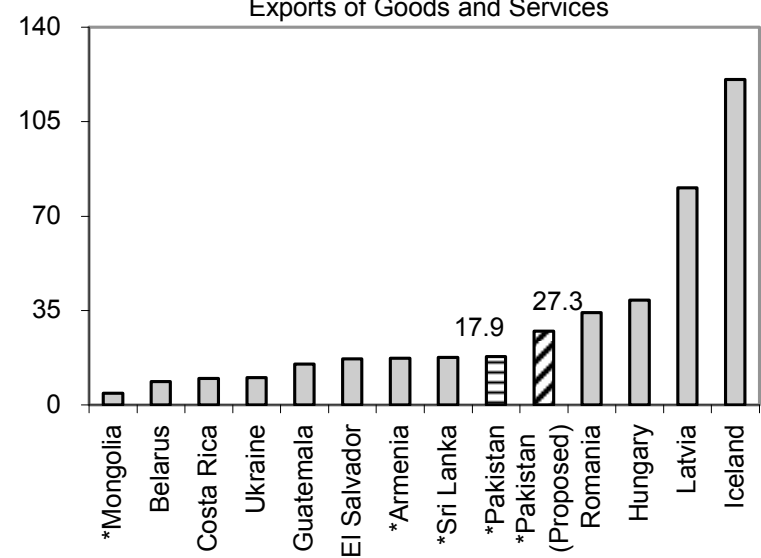

B. Debt Service to the Fund in Percent of Exports of Goods and Services

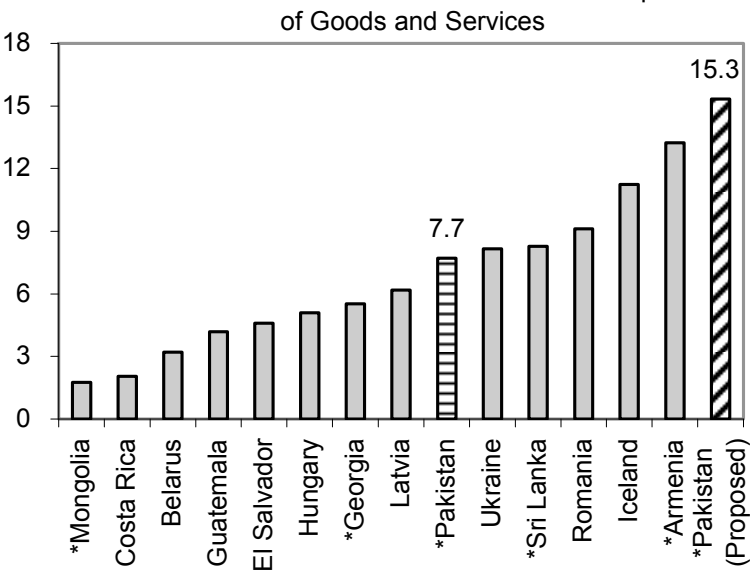

C. Debt Service to the Fund in Percent of Total External Debt Service

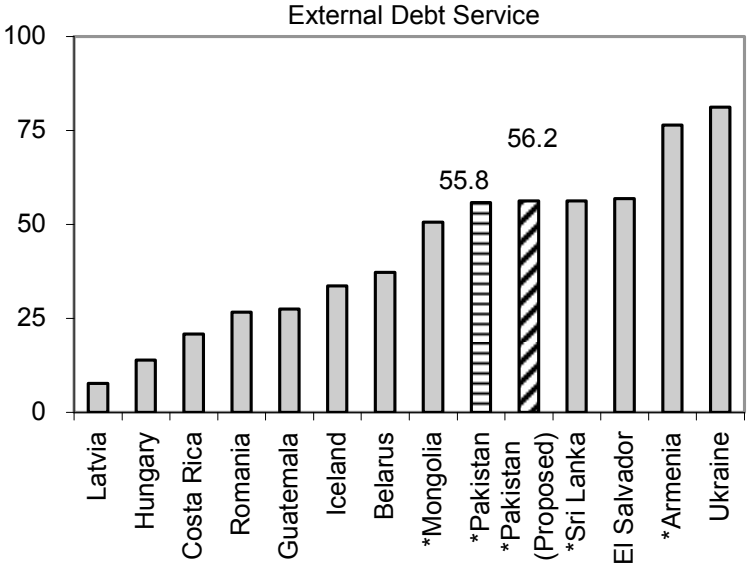

Source: Pakistan authorities and IMF staff estimates, and World Economic Outlook.

1/ Asterisks indicate PRGF eligible countries. 
Table 3. Pakistan - Impact on GRA Finances

as of $07 / 20 / 2009$

\begin{tabular}{|c|c|}
\hline Liquidity measures & \\
\hline One-year Forward Commitment Capacity (FCC) (in millions of SDRs) $1 /$ & $91,300.0$ \\
\hline Impact on FCC of Pakistan's Augmentation & $(2,067.4)$ \\
\hline Prudential measures & \\
\hline Fund GRA credit outstanding to Pakistan (percent of current precautionary balances) 2 / & 48.0 \\
\hline Pakistan's annual GRA charges (percent of Fund's residual burden sharing capacity) 3/ & 425.7 \\
\hline Fund GRA credit outstanding to Pakistan (percent of total GRA credit outstanding) $2 /$ & 13.8 \\
\hline Memorandum items & \\
\hline Fund's precautionary balances (in millions of SDRs) 4/ & $7,093.0$ \\
\hline Fund's residual burden sharing capacity (in millions of SDRs) $5 /$ & 20.0 \\
\hline Fund GRA credit outstanding to five largest debtors (percent of total GRA credit outstanding) 2/ & 84.1 \\
\hline Sources: Finance Department & \\
\hline $\begin{array}{l}\text { 1/ The Forward Commitment Capacity is a measure of the resources available for new financial com } \\
\text { year, equal to usable resources minus undrawn balances under existing arrangements plus repurch } \\
\text { minus a prudential balance. The FCC is determined on basis of available quota resources and resou } \\
\text { bilateral borrowing arrangements. In addition, the Fund has access to SDR } 34 \text { billion under the NAB } \\
\text { 2/ Reflects GRA credit outstanding, including Pakistan's August purchase upon approval of the augn } \\
3 \text { / The ratio is estimated using GRA charges for fiscal year 2009/10. } \\
\text { 4/ As of end-April } 2009 \text {. } \\
5 \text { / Burden-sharing capacity is calculated based on the floor for remuneration at } 85 \text { percent of the SD } \\
\text { burden-sharing capacity is equal to the total burden-sharing capacity minus the portion being utilized } \\
\text { and takes into account the loss in capacity due to nonpayment of burden sharing adjustments by me }\end{array}$ & $\begin{array}{l}\text { ing } \\
\text { ngements. } \\
\text { review. } \\
\text { ual } \\
\text { arges }\end{array}$ \\
\hline
\end{tabular}

\section{ASSESSMent}

\section{The proposed augmentation of access under the SBA involves substantial}

financial risks to the Fund. At the time of approval of the program, the level of access was already large in terms of Pakistan's economy and debt servicing capacity, and significant in relation to the Fund's resources. Access will be increased significantly under the augmentation. About half of the augmentation could be used to finance near-term government spending until resources pledged by donors in April are disbursed later in the fiscal year. Program implementation to date has been mixed, complicated by a difficult security and political situation. Debt service to the Fund will become a significant fiscal burden, and is particularly high relative to reserves, which remain vulnerable to weaker exports, remittances, FDI, and possible delays in donor disbursements. Under the current program, there have been delays in implementation of key structural fiscal reforms, in particular, measures to raise revenues and to address (with World Bank and ADB technical and financial support) the rising costs of electricity subsidies. The revised program framework sets out a number of corrective actions to address these delays and renew fiscal reform efforts. Their prompt implementation will be critical to timely repayment and averting prolonged financial engagement with the Fund. 


\section{Statement by the Staff Representative on Pakistan}

August 7, 2009

1. This statement provides information on developments since the staff report was circulated to the Executive Board on July 29, 2009. It does not alter the thrust of the staff appraisal.

2. Balance-of-payments data indicate a slightly larger current account deficit for $2008 / 09$ than estimated in the staff report. The estimated difference is $\$ 0.3$ billion or 0.2 percent of GDP.

3. Gross official reserves amounted to $\$ 8.2$ billion on August 3, 2009, compared with \$9.1 billion at end-June 2009. The decline relative to end-June is explained by several factors. In July, reserves were affected by official payments (\$312 million), a reduction in net forward position ( $\$ 100$ million), and larger provision of foreign exchange by the State Bank of Pakistan (SBP) for oil import payments than in June (due to higher prices), and some pre-financing for petroleum product imports, as the SBP announced that it would stop providing foreign exchange for petroleum product imports from August 1.

4. Efforts are being made to strengthen the enforcement of limits on provincial government use of SBP overdrafts, a key reason for the fiscal overrun in the last quarter of 2008/09. The Ministry of Finance has initiated discussions with the provinces to agree on new limits on Ways and Means Advances. Pending the establishment of new limits, it has instructed the SBP to enforce strictly existing limits and not to honor checks in excess of these limits. Further, regular monthly meetings will be held between the federal and provincial finance ministries to agree on provincial expenditure and its financing. Discussion on a schedule of reduction of the overdraft stock between the provinces, the Ministry of Finance, and the SBP has begun.

5. The National Electric Power Regulatory Authority (NEPRA) Act was amended by a Presidential Ordinance on July 31, 2009_a prior action. The amendment puts in place:

(i) monthly determination by the NEPRA of the fuel adjustment surcharge in line with international fuel prices and automatic implementation upon determination; and (ii) quarterly determination of overall electricity tariffs by NEPRA and notification of the adjusted tariffs usually within 15 days unless the government requests a review of the calculations made, in which case the maximum period before notification is made will be 33 days. While this amendment varies from what was envisaged under the prior action, staff is of the view that the amendments, nevertheless, broadly achieve the intended objective. World Bank staff agrees that the amendments are largely consistent with the agreed plan for eliminating electricity tariff differential subsidies. However, for the amended NEPRA Act to become effective on a permanent basis, these amendments will need to be approved by parliament before the Presidential ordinance expires after four months. 


\section{IMF Completes Second Review Under Stand-By Arrangement for Pakistan and Increases Financial Support to US\$11.3 billion}

The Executive Board of the International Monetary Fund (IMF) today completed the second review of Pakistan's economic performance under a program supported by a Stand-By Arrangement (SBA).

To help the country address increased balance of payment needs, the Board also approved an augmentation of access by an amount equivalent to SDR 2,067.4 million (about US\$3,236.3 million), bringing the total financial support to an amount equivalent to SDR 7,235.9 million (about US\$11,327.0 million), equivalent to 700 percent of Pakistan's quota or 6.3 percent of its GDP.

The Board also approved the extension to end 2010 of the arrangement, originally approved on November 24, 2008 (see Press Release No. 08/303).

The completion of the review enables the immediate disbursement of an amount equivalent to SDR 766.7 million (about US\$1,200.2 million), bringing total disbursements under the program to an amount equivalent to SDR 3,402.6 million (about US\$5,326.5 million). The Board agreed that a portion of the augmented access could be used to finance priority spending until the disbursements of donor support pledged for 2009/10 are received.

Pakistan will also benefit from the proposed allocation of Special Drawing Rights (SDRs), which, once approved, will supplement its reserves.

The Executive Board also approved Pakistan's request for a waiver for the non-observance of two end-June 2009 structural performance criteria on submission to parliament of legislative amendments to (i) enhance the effectiveness of the State Bank of Pakistan in banking supervision; and to (ii) harmonize the income tax and sales tax laws and reduce exemptions for both taxes. The Executive Board also approved Pakistan's request for a waiver for nonobservance for the end-June quantitative performance criterion on the fiscal deficit, which 
according to preliminary information was missed by an amount equivalent to 0.9 percent of GDP.

Following the Executive Board's discussion on Pakistan, Mr. Murilo Portugal, Deputy Managing Director and Acting Chair, stated:

"Pakistan's economy has continued to stabilize. Reforms in the financial sector and the foreign exchange market have been progressing, and steps have been taken to strengthen the social safety net. These achievements are appreciable, considering the security developments that resulted among others in the large number of internally displaced persons (IDPs), the global economic recession, and the difficult domestic political environment.

"The end-2008/09 fiscal deficit target was missed, due partly to unforeseen security- and IDP- related expenditures but also to excessive spending by provinces and revenue shortfalls, owing partly to delayed implementation of tax reforms. The unresolved energy sector problems have continued to undermine Pakistan's growth potential and burden public finances.

"A durable solution to the problem of low tax revenue should start with the early implementation of VAT and the ongoing tax administration reform. These reforms will make the economy less vulnerable, provide the steady flow of resources needed to reduce poverty and develop basic infrastructure, and strengthen the government's ability to deal with the pressing needs of the population, which are now compounded by the large number of IDPs.

"The recent agreement with World Bank and Asian Development Bank staffs on the electricity sector reform signals a desire to address the deep-seated problems in that sector, including the resolution of the intercorporate circular debt, which burdens the energy sector enterprises, and the elimination of tariff differential subsidies within a year from now, making additional fiscal resources available for priority spending.

"The macroeconomic outlook for 2009/10 remains difficult, and the external position is subject to considerable downside risks. The donor support pledged in Tokyo and the augmentation of access under the IMF-supported Stand-By Arrangement by about $\$ 3.1$ billion will help mitigate these risks and enable the implementation of the government's fiscal program; however, this financing is temporary and should be used as a bridge until the revenue reforms bear fruit.

"The 2009/10 budget aims to provide adequate space for priority spending. This includes spending on IDPs, support for poor households, and other well-targeted social spending. 
"Monetary policy should remain vigilant about preventing a resurgence of inflation. The relaxation of the fiscal policy stance, electricity tariff increases, and the rebound in oil prices will add to inflationary pressures that monetary policy needs to combat.

"The increased flexibility of the exchange rate and the timely elimination of the State Bank of Pakistan's provision of foreign exchange for imports of diesel and other refined products will improve the functioning of the foreign exchange market, make Pakistan's economy more resilient to external shocks, and will contribute to further strengthening of its international reserves position.

"The accelerated reforms to strengthen central bank independence and the legislative amendments to increase its supervisory powers will enhance the monetary policy framework and help strengthen the banking system," Mr. Portugal said. 


\section{Statement by Jafar Mojarrad, Executive Director for Pakistan and Ehtisham Ahmad, Senior Advisor to Executive Director August 7, 2009}

Pakistan continues to face significant economic and political challenges, including a heightening of the war on terror with the recent military actions in the North West of the country, leading to 3 to 4 millions internally displaced persons (IDPs). Nonetheless, the Fund program has been extraordinarily successful in stabilizing the economy and preparing the foundations for sustainable growth. In broad terms, there has been an overall net fiscal adjustment of around 2.5 percent of GDP, in the midst of an economic and political crisis, stabilization of the rupee, albeit at a 30 percent lower level, and sharp reduction in inflation. The Pakistan authorities are grateful to management and staff for their exemplary dedication, understanding and support, and to the Board for approving the program.

The Pakistan authorities are also greatly indebted to the Friends of Pakistan for their pledges for support in the Tokyo meeting in April, organized by the Japanese authorities and the World Bank.

\section{The background}

Immediately preceding the program, Pakistan faced a fiscal deficit of 7.6 percent of GDP for 2007/8, incipient inflationary pressures, and reserves that declined from around US\$14.5 billion in October 2007 to around US\$3.5 billion in October 2008. It was recognized that an underlying cause of the weakness was the low level of domestic resource mobilization.

It may be useful to keep in mind that a shift from trade taxes towards the GST was undertaken in the late 1990s. However, the GST never compensated for the revenues lost from trade taxes as had been intended, as problems with refunds and an incomplete revenue base forced the effective removal of the main sectors from the GST chain. Thus, it was not surprising that tax revenues tanked from around 14 percent of GDP in the late 1990 s, to 9.8 percent in $2007 / 8$.

While a project to reform the tax administration, the Federal Board of Revenue (FBR), was launched in 2001, with significant World Bank and DFID support, the situation in August 2008 was that a functional administration had not been implemented, the process of business process engineering had not been initiated, and the audit capability had been virtually abandoned. 


\section{Major actions taken and problems faced}

One of the most significant measures taken by this administration in September 2008 was the full pass-through of energy and food prices at their international peak, albeit with a very weak social safety net and at considerable political risk. Although it added to the incipient inflationary pressures, the price adjustment significantly reduced the fiscal deficit, which was 7.6 percent of GDP in 2007/8, by at least 2.4 percentage points, even though the revenue performance was indeed very disappointing, for reasons that we discuss later.

On the structural side, the government has placed a reform of the FBR at the center of its medium term strategy, together with an emphasis on transforming the ineffective General Sales Tax (GST) into a proper VAT. In order to rescue the moribund FBR-reform project, the government identified former FAD staff member, Mr. Silvani, to provide a roadmap, and is grateful to the World Bank and DFID for financial and logistical support for this effort. Successive missions by the Silvani team in September and December 2008 led to the reiteration of the strategy based on an integrated functional administration, with streamlined business processes and audit, and supported by modern IT systems. Early in 2009, the government moved to implement an integration of the administration of excises and sales taxes (run by the customs and excise service) and the income taxesbut this was opposed by service cadres. In particular, the customs and excise service issued a challenge in the Islamabad High Court on the grounds that integration might adversely affect the career prospects of its member. The Court issued a stay-order in April that temporarily reversed the integration.

The tax policy agenda remains focused on the full VAT, and should generate an additional 3 percentage points of GDP in the next few years. Expansion of the base is a function of the enhancements in the FBR and confidence that a revamped refund system will not lead to a reduction in revenues, or pose a constraint for exporters. A comprehensive treatment of the base also has to take into account the constitutional jurisdiction of the provinces over the taxation of services. The government will address this issue with multilateral assistance, in early September, leading to the drafting of a new VAT law. Inter-jurisdictional issues will also come up before the quinquennial Finance Commission that will begin its work in September.

The 2008/9 budget envisaged an increase in the tax/GDP effort to 10.3 percent (largely based on an increase in the GST rate and some additional excises) and the program in October envisaged an additional 0.5 percent of GDP for the remainder of the year on account of tax administration measures. In fact, the outcome at 8.8 percent of GDP was lower than the level achieved in 2007/8. Raising the rate structure of the current GST without addressing the underlying problems has proved to be ineffective. The poor tax revenue performance during 2008/9 was partly due to the compression of imports, fall in 
activity levels, but also the non-cooperation by the tax administration officials, that was not resolved before the budget was passed at the end of June.

With declining revenues, the fiscal adjustment was achieved by a tight control over central government current and capital spending in the wake of the program. The targeted budget deficit of 4.3 percent of GDP, a quantitative performance criterion, would likely have been met, but for two exogenous factors. The first was the military action against Al Qaeda in the North, leading to over 3 million IDPs that had to be housed and fed, and for reconstruction in the war zone. The second is unexpected borrowing by provinces in the last few days of the fiscal year, in excess of normal ways and means advances. The provinces explained that they made their budgets on the expectation that their share of total revenues budgeted (around Rs. 1250 billion) would materialize. A waiver for the non-observance of the fiscal deficit target is sought.

In order to address the provincial borrowing issue, the government has now constituted a Committee to Coordinate Interprovincial Finances that will meet monthly in order to advise the provinces of the need to make adjustments during the year. The provinces have also been informed that the ways and means limits, specified in the constitution (hence additional legal cover is not needed), will be strictly enforced and that their cheques will be bounced if needed. The elimination of the stock of provincial arrears depends on the assignments and transfers, especially for the deficit provinces (NWFP and Baluchistan) that are also the focus of military action. These issues will need to be addressed in conjunction with the Finance Commission.

A tight monetary policy, together with the floating of the exchange rate, which has adjusted by almost 30 percent during the year, has led to a replenishment of reserves, as well as sharp fall in headline and core inflation.

\section{The Budget and Structural Benchmarks}

The main revenue initiative for 2009/10 was the bold move towards a specific amount for a carbon tax (now called the petroleum levy), set to yield around 1.2 percent of GDP. Even though the measure attracted severe political criticism, effective social safety nets to compensate the poor are not yet in place, and the Supreme Court temporarily struck it down, the government persevered and the measure remains in place, albeit with a different legal basis and name. This measure has insulated the budget from the recent rise in petroleum prices towards US\$75, and a move in the tax ratio from 8.8 to 10.6 in 2009/10 appears assured.

Although the government had originally agreed to a structural benchmark to remove some zero-rating of sectors from the GST by June 2009, it makes no sense to do this

before the tax administration is ready to administer automatic refunds. It is also not very 
rational to harmonize the income tax and a faulty GST law that is to be abandoned when a new VAT law is implemented in 2010. Proposals for harmonization of the administrative aspects to facilitate joint management of the income and sales taxes also could not be introduced in the budget, given the court order preventing integration. Now that there has been an agreement between the government and the services to safeguard seniority and benefits, it is expected that the court order will be rescinded, enabling the administrative harmonization to go forward. Despite the disappointment with the "inadequate" revenue outcomes, which we share with Fund staff, it is difficult to see what else the government could have done under these circumstances. Consequently, waivers are sought for structural benchmarks relating to the harmonization of the GST and income tax laws and removal of zero-rated sectors from the GST.

There are some options for raising excises on luxury goods, but there is a limit beyond which such measures generate serious distortions. The authorities have resisted calls to return to the pre-2000 reliance on import duties. The new budget also envisages taxation of vested landed interests and other "sacred cows" that have biased tax policy in Pakistan for decades. In particular, key measures enacted for 2009/10 include the taxation of capital gains on property transactions, and imposition of excises on certain services with the agreement of provinces - anticipating the introduction of the full VAT.

A major problem area remains the generation and distribution of electricity for resumed exports, growth and consumption. In the late 1990s, in order to encourage investment, the then government on the advice of the ADB and the World Bank moved to a model of privatized or autonomous power-generating companies linked to a national grid. Regrettably, there was almost no investment in the past decade, and relatively weak governance and oversight has led to major quasi-fiscal liabilities for the government. This model also led to an absence of incentives for the power producers to operate efficiently. This also led to the creation of circular debt, and attempts to break this chain have led to successive discoveries of additional liabilities, leading to a need to adjust electricity prices.

Earlier this year, the Bank and ADB estimate was that a 4 percent increase would suffice to address the issue of arrears in the power sector. In May, it transpired that the adjustment might be closer to 16 percent, and by July this figure had risen to 31 percent. The government has adopted a mechanism to ensure that all changes in costs are passed through to prices, with the amendment of the NEPRA act through an ordinance last week, as the National Assembly is not in session. This measure has resolved the "flow" problem, given changes in international prices, mainly of fuel oil. As far as the stock of liabilities is concerned, the government remains committed to address the issue through gradual price rises, as the supply of electricity improves (there have been additional problems with Mangla Dam). It is not politically feasible undertake a one-shot price increase, following the introduction of the carbon tax (which also led to an increase in 
prices), and an inadequate social safety net. This strategy has also been agreed with the $\mathrm{ADB}$ and the World Bank.

The safety net remains critical to bolster political support for the program and the government. It is also essential to ensure that the IDPs are effectively provided for. As the program only includes an adjuster for external IDP-related grants, the budgetary allocation for this essential spending remains modest - the expectation being that friendly governments will provide the needed additional support.

\section{Monetary and exchange policies}

The tight monetary policy has achieved the twin objectives of restoring confidence in the exchange rate, now freely determined, and bringing down inflation sharply. NFA and NDA quantitative performance targets were met. There is very weak private demand for credit. Although there is now considerable pressure from the markets on SBP to reduce the policy rate, given that the KIBOR rate is now well below the policy rate and growth is anemic, the SBP has been cautious in cutting the rates given the uncertainty in extent and timing of multilateral flows. However, with the sustained decline in core-inflation, there is scope for a cut in the policy rate - this, however, will be coordinated with the Fund staff.

The government remains committed to the independence of the State Bank, and legislation strengthening the SBP Law has been prepared, and is expected to be submitted to Parliament in the September session, as is legislation to strengthen banking supervision. It was not possible to submit this legislation during the very crowed budget session in June. Waivers are requested in connection with these structural benchmarks.

Progress towards the establishment of a Treasury Single Account continues, preventing an accumulation of new balances outside the Federal Consolidated Fund. However, the existing deposits need to be verified depending on a survey to be conducted on the sources and legal bases of such accounts. The elimination of these accounts has to be coordinated with the State Bank in light of the health of the banking sector in light of the increasing NPLs.

\section{Going forward}

Although reserves accumulation has exceeded the program target, moving the imports of petroleum prices to the free market has placed additional pressure on the exchange rate and reserves. Additional risks emanate from the uncertainty associated with remittances, that have performed better than expected, and exports that have really suffered over the past year. For these reasons, the Pakistan authorities request the augmentation of access by 200 percent of quota. 
Given the geopolitical importance of "social stabilization" in Pakistan, the Friends of Pakistan have generously agreed to support the sizable investment and reconstruction needs of the country. Given the likely sequencing of this support, the Government requests that a part of the augmentation be used temporarily for budgetary support, to be reimbursed to the SBP as Tokyo monies come in.

The authorities appreciate the support of the Fund team that has focused on the main objectives and measures in a flexible manner. They also appreciate the very successful outreach by MCD management. The Pakistan authorities look forward to the continued support of the Board, Management and Staff. 